$\underline{\text { Original Article }}$

\title{
Epidemiology of Methicillin-Resistant Staphylococcus Aureus in Arab Countries of the Middle East and North African (MENA) Region
}

\author{
Hussam Tabaja ${ }^{1}$, Joya-Rita Hindy ${ }^{1}$ and Souha S. Kanj ${ }^{2}$. \\ ${ }^{1}$ Mayo Clinic, Division of Infectious Diseases, Department of Internal Medicine, Rochester, Minnesota, \\ USA. \\ ${ }^{2}$ American University of Beirut Medical Center, Division of Infectious Diseases, Department of Internal \\ Medicine, Beirut, Lebanon.
}

Competing interests: The authors declare no conflict of Interest.

\begin{abstract}
Available data suggest a high burden of methicillin-resistant staphylococcus aureus (MRSA) in Arab countries of the Middle East and North Africa (MENA). To review the MRSA rates and molecular epidemiology in this region, we used PubMed search engine to identify relative articles published from January 2005 to December 2019. Great heterogeneity in reported rates was expectedly seen. Nasal MRSA colonization ranged from $2 \%-16 \%$ in Gulf Cooperation Council (GCC), 1-9\% in the Levant, and 0.2\%-9\% in North African Arab states. Infective MRSA rates ranged from 9\%-38\% in GCC, $28 \%-67 \%$ in the Levant, and $28 \%-57 \%$ in North African states. Studies demonstrated a wide clonal diversity in the MENA. The most common molecular types belonged to 5 clonal complexes (CC) known to spread worldwide: CC5, CC8, CC22, CC30, and CC80. The most prevalent strains had genotypes related to the European community-acquired MRSA (CA-MRSA), Brazilian/Hungarian hospital-acquired MRSA (HA-MRSA), UK-EMRSA15 HA-MRSA, and USA300 CA-MRSA. Finally, significant antimicrobial resistance was seen in the region with variation in patterns depending on location and clonal type. For a more accurate assessment of MRSA epidemiology and burden, the Arab countries need to implement national surveillance systems.
\end{abstract}

Keywords: MRSA; Methicillin-Resistant Staphylococcus aureus; Arab countries; Middle East; North African.

Citation: Tabaja H., Hindy J.R., Kanj S.S. Epidemiology of methicillin-resistant staphylococcus aureus in arab countries of the middle east and north african (MENA) region. Mediterr J Hematol Infect Dis 2021, 13(1): e2021050, DOI: http://dx.doi.org/10.4084/MJHID.2021.050

This is an Open Access article distributed under the terms of the Creative Commons Attribution License (https://creativecommons.org/licenses/by-nc/4.0), which permits unrestricted use, distribution, and reproduction in any medium, provided the original work is properly cited.

Correspondence to: Souha S. Kanj, MD, FACP, FIDSA, FRCP, FESCMID, FECMM. Professor of Medicine. Head, Division of Infectious Diseases, Chairperson, Infection Control Program, American University of Beirut Medical Center, P.O. Box 11-0236 Riad El Solh 1107 2020, Beirut, Lebanon. Tel: 961-1-350000; Fax: 961-1-370814. E-mail: sk11@aub.edu.lb

Introduction. The last few decades have witnessed an increasing interest in the molecular epidemiology of methicillin-resistant Staphylococcus aureus (MRSA). Various techniques have been utilized to decipher the MRSA genotype, which could provide insight into a strain's resistance capacity and virulence. It also provides insight into their origin of spread and helps implement proper infection control measures. ${ }^{1}$

To date, MRSA is the most frequently identified antibiotic-resistant microbe in many regions, including East Asia, the Middle East, North Africa, Europe, and the Americas. ${ }^{2}$ Available data suggest a high burden of MRSA in Arab countries of the Middle East and North Africa (MENA). ${ }^{3,4}$ However, the Arab world lacks both regional and national surveillance systems. Instead, the current literature is derived from fragmented singlecentered research. Moreover, no review summarizing such findings is yet available. Therefore, the purpose of 
our paper is to examine the current literature on the epidemiology of MRSA in Arab countries of the MENA region.

\section{Definitions.}

The Middle East and North African Region: According to the World Bank, the MENA region comprises 21 member states. The Arab member states include the Kingdom of Saudi Arabia (KSA), Unites Arab Emirates (UAE), Kuwait, Qatar, Bahrain, Oman, Yemen, Syria, Lebanon, Jordan, Palestine (west bank and Gaza strip), Iraq, Egypt, Tunisia, Algeria, Morocco, Yemen, and Libya. $^{5}$

MRSA molecular typing techniques: Currently, there are four molecular typing techniques, including pulsed-field gel electrophoresis (PFGE), Multilocus sequence typing (MLST), staphylococcal cassette chromosome mec (SCCmec) typing, and S. aureus protein A gene (spa) typing.

Pulsed-field gel electrophoresis: It is considered the reference standard for MRSA typing. Uses restriction enzyme SmaI to digest purified chromosomal DNA followed by agarose gel electrophoresis. A unique pattern is created and analyzed using the Dice coefficient and unweighted pari-group matching analysis (UPGMA). Thus, it is the most discriminative method and the best for investigating hospital outbreaks. Nonetheless, efforts to harmonize PFGE protocols have failed, which hinders inter-laboratory comparability. ${ }^{1}$ Therefore, it is not useful when comparing isolates from different centers. ${ }^{6}$ Multilocus sequence: It is based on sequence analysis of 7 housekeeping genes (arcC, aroE, glpF, gmk, pta, tpi, yqiL). Each unique sequence within a gene is designated as an allele. When combined, the 7-allele pattern behaves as an allelic profile or sequence type (ST) that defines the MRSA isolate. For example, all isolates with the allelic profile 7-6-1-5-8-8-6 are clones and this sequence is identified as ST5. However, this method is laborious and time-consuming. ${ }^{1}$

A database for MLST typed isolates identified and reported globally exists through http://www.pubmlst.org Staphylococcal Cassette Chromosome mec: SCCmec is a mobile genetic element that harbors mecA gene. Different typing methods exist, utilizing polymerase chain reaction (PCR) technology. There are five main types, SCCmec I to V, ${ }^{1}$ but overall, 13 types have been reported (type I-XIII), some of which have subvariants. ${ }^{7}$ Staphylococcus aureus Protein A Gene Typing: This is a single-locus DNA sequence typing of the polymorphic region $\mathrm{X}$ of spa gene. The typing reveals a specific pattern of repeats which is then used to deduce the spa type. It is simpler than the tedious MLST typing. It has good discriminatory power that lies between PFGE and MLST. Although individual laboratories use "in-house" sequencing platforms, a dedicated software (Ridom
StaphType software) that analyzes sequencing patterns from different laboratories exists and is accessible to the public. This software helps ensure interlaboratory comparability. A laboratory's typing data can be uploaded and synchronized into the database via a central server available at http://www.spaserver.ridom.de. The database currently has 20,056 spa types reported, representing 444,798 total strains from 146 countries. ${ }^{1}$

Clonal Complex: The primary method of determining clonal complex (CC) is based on MLST. Individual isolates with an identical sequence in at least 5 out of 7 housekeeping genes are grouped into a $\mathrm{CC}$. The ancestor of a $\mathrm{CC}$ is the ST with the highest single-locus variants. Therefore, each ST will belong to a single CC, but each CC can have several STs with five identical housekeeping genes. For example, ST228 and ST5 both belong to CC5. ${ }^{1}$

Due to a higher discriminatory power, several spa types can belong to the same ST. A unique spa-type can be shared among several STs but can belong to only 1 $\mathrm{CC}$. Therefore, clustering analysis based on spa typing is also feasible (i.e., spa-CC). Supplementary Table 1 reports the relative global frequencies of common spa types.

MRSA Clone Nomenclature: The nomenclature of MRSA strains is determined by the combination of ST and SCCmec type. For example, ST239-SCCmec-III is colloquially known as Brazilian/Hungarian clone. Table 1 lists some of the major clones identified globally. Some clones can have more than 1 colloquial name.

For a more detailed description of MRSA molecular typing, we refer our readers to a publication by Deurenberg et al.. ${ }^{1}$ Additionally, a comprehensive guide describing the different MRSA clones is available. ${ }^{8}$ Finally, Supplementary Figure 1 illustrates the interconnection of different typing methods.

Panton-valentine leucocidin (PVL) and toxic shock syndrome-toxin 1 (Tst1): The lukF/S-PV and TST genes encode $P V L$ and $T s t 1$, respectively, which are virulence factors found in MRSA. ${ }^{9}$ More specifically, $[P V L+]$ strains can cause leukocyte destruction and tissue necrosis and are frequently associated with skin and soft tissue infections and necrotizing pneumonia. ${ }^{10} P V L$ is commonly found in CA-MRSA strains and rarely present in HA-MRSA strains. ${ }^{8}$

Community-acquired MRSA (CA-MRSA) vs. Hospitalacquired MRSA (HA-MRSA): Distinguishing between CA-MRSA and HA-MRSA has traditionally been based on genetic makeup. ${ }^{1}$ CA-MRSA is typically associated with SCCmec types IV/V while HA-MRSA is associated with types I/II/III. However, CA-MRSA carrying SCCmec type I, II, and III and HA-MRSA carrying type IV have been identified questioning the distinction based on SCCmec alone. ${ }^{1}$ 
Table 1. Major MRSA Clones Encountered Globally.

\begin{tabular}{|c|c|c|c|c|c|c|}
\hline Clone & MLST & ST & $\mathbf{C C}$ & SCCmec & Spa & $\begin{array}{l}\text { Geographic areas } \\
\end{array}$ \\
\hline UK-EMRSA-3 & $1-4-1-4-12-1-10$ & 5 & 5 & I & $\begin{array}{l}\mathbf{t 0 0 3}, \mathbf{t 0 0 2}, \mathrm{t} 001, \mathrm{t} 010 \\
\mathbf{t 0 4 5}, \mathrm{t} 053, \mathrm{t} 062, \mathrm{t} 105 \\
\mathrm{t} 178, \mathrm{t} 179, \mathrm{t} 187, \mathrm{t} 214 \\
\mathrm{t} 311, \mathrm{t} 319, \mathrm{t} 389, \mathrm{t} 443\end{array}$ & $\begin{array}{l}\text { Af, Arg, Aus, Au, Bel, Bra, Can, Chi, China, } \\
\text { Cro, Cyp, Cze, Den, Det, Es, Fin, Fr, Ga, Ger, } \\
\text { Gr, HK, Hun, Ice, Iran, Ire, Isr, It, Jap, Ma, } \\
\text { Mal, Mart, Min, Nets, NZ, Nor, Pol, Por, Ro, } \\
\text { SA, Slo, Sp, Swe, Swit, Tai, UK, USA }\end{array}$ \\
\hline New York/Japan & $1-4-1-4-12-1-10$ & 5 & 5 & II & $\begin{array}{l}\mathbf{t 0 0 3 ,} \mathbf{t 0 0 2}, \mathrm{t} 001, \mathrm{t} 010 \\
\mathbf{t 0 4 5}, \mathrm{t} 053, \mathrm{t} 062, \mathrm{t} 105 \\
\mathrm{t} 178, \mathrm{t} 179, \mathrm{t} 187, \mathrm{t} 214 \\
\mathrm{t} 311, \mathrm{t} 319, \mathrm{t} 389, \mathrm{t} 443\end{array}$ & $\begin{array}{l}\text { Aus, Aust, Belg, Can, Cro, Den, Fin, Fra, } \\
\text { Ger, HK, Hun, Ire, Jap, Kor, Mex, Por, Sin, } \\
\text { Swe, Tai, Uru, UK, USA }\end{array}$ \\
\hline Paediatric & $1-4-1-4-12-1-10$ & 5 & 5 & IV & $\begin{array}{l}\mathbf{t 0 0 1}, \mathbf{t 0 0 2}, \mathbf{t 0 0 3}, \mathrm{t} 010, \\
\mathbf{t 0 4 5}, \mathrm{t} 053, \mathrm{t} 062, \mathrm{t} 105 \\
\mathrm{t} 178, \mathrm{t} 179, \mathrm{t} 187, \mathrm{t} 214, \\
\mathrm{t} 311, \mathrm{t} 319, \mathrm{t} 389, \mathrm{t} 443\end{array}$ & $\begin{array}{l}\text { Alg, Arg, Aust, Bra, Col, Den, Fr, Kor, Nor, } \\
\text { Pol, Por, Sp, Swe, Uru, UK, USA }\end{array}$ \\
\hline South German & $1-4-1-4-12-24-29$ & 228 & 5 & I & $\begin{array}{l}\text { t041, t023, t201, t188, } \\
\text { t001 }\end{array}$ & $\begin{array}{l}\text { Aust, Aus, Cro, Ger, Hun, Isr, It, Malta, Par, } \\
\text { Slo, Swit }\end{array}$ \\
\hline $\begin{array}{l}\text { Brazilian/Hungarian } \\
\text { HA-MRSA }\end{array}$ & $2-3-1-1-4-4-3$ & 239 & 8 & III & $\begin{array}{l}\mathbf{t 0 3 7}, \mathrm{t} 030, \mathrm{t} 234, \mathrm{t} 387, \\
\mathrm{t} 388\end{array}$ & $\begin{array}{l}\text { Arg, Aust, Aus, Bel, Bul, Can, Chi, China, } \\
\text { Cro, Cze, Den, Egy, Fr, Ger, Gr, Ice, In, Iran, } \\
\text { Ire, Isr, It, Latvia, Ma, Mal, Min, Nets, NZ, } \\
\text { Nor, Pol, Ro, SA, Sp, Swe, Swit, Tai, Tur, } \\
\text { Ug, UK, USA, China }\end{array}$ \\
\hline Iberian & $3-3-1-12-4-4-16$ & 247 & 8 & $\mathrm{I}$ & $\begin{array}{l}\mathbf{t 0 0 8}, \mathrm{t} 051, \mathrm{t} 052, \mathrm{t} 054, \\
\mathrm{t} 200, \mathrm{t} 064\end{array}$ & $\begin{array}{l}\text { Aust, Bel, Cro, Cze, Den, Fin, Fr, Ger, It, } \\
\text { Nets, Pol, Por, Slo, Sp, Swe, Swit, UK, USA }\end{array}$ \\
\hline USA 300 CA-MRSA & $3-3-1-1-4-4-3-8$ & 8 & 8 & IV & t008 & $\begin{array}{l}\text { Aust, Ger, Iraq, Ire, Sp, Swit, UK, USA, Jap, } \\
\text { HK }\end{array}$ \\
\hline $\begin{array}{l}\text { UK-EMRSA-15 HA- } \\
\text { MRSA }^{\text {B }}\end{array}$ & $7-6-1-5-8-8-6$ & 22 & 22 & IV & $\begin{array}{l}\mathbf{t 0 3 2}, \mathbf{t 0 2 2}, \mathbf{t 0 0 5} \\
\mathbf{t 2 2 3}, \mathrm{t} 309, \mathrm{t} 310, \mathrm{t} 417, \\
\mathrm{t} 420\end{array}$ & $\begin{array}{l}\text { Aus, Bel, Cro, Can, Den, Det, Ger, Ice, Ire, It, } \\
\text { In, Mal, Malta, Min, Nets, NZ, Nor, Por, SA, } \\
\text { Sp, Swe, UK, }\end{array}$ \\
\hline $\begin{array}{l}\text { Southwest pacific } \\
\text { CA-MRSA }\end{array}$ & $2-2-2-2-6-3-2$ & 30 & 30 & IV & $\begin{array}{l}\mathbf{t 0 1 2}, \mathbf{t 0 2 1}, \mathrm{t} 019, \mathbf{t 0 1 8}, \\
\text { t318, t338, t138, t276, } \\
\text { t268, t391 }\end{array}$ & $\begin{array}{l}\text { Aust, Ger, HK, Ire, Ku, La, NZ, Sca, Swit, } \\
\text { Tai, UK, USA }\end{array}$ \\
\hline $\begin{array}{l}\text { European CA- } \\
\text { MRSA }^{\mathrm{A}}\end{array}$ & $\begin{array}{l}1-3-1-14-11-51- \\
10\end{array}$ & 80 & 80 & IV & $\mathbf{t 0 4 4 ,}$ t416 & $\begin{array}{l}\text { Alg, Aus, Au, Bel, Bul, Chi, Cro, Cyp, Cze, } \\
\text { Den, Det, Egy, Fin, Fr, Ger, Gr, Hun, Ice, } \\
\text { Iran, Ire, It, Isr, Ku, Malta, Nets, Nor, Ro, Sp, } \\
\text { Swe, Swit, Tur, UK, Nets, Cam, Moz }\end{array}$ \\
\hline
\end{tabular}

Data adopted from Deurenberg et al (5), Monecke et al (6), http://www.spaserver.ridom.de, and http://www.pubmlst.org. Bolded spa types are the most commonly reported types globally (refer to supplementary table 1).

Arg: Argentina; Af: Afghanistan; Alg: Algeria; Aust: Australia; Au: Autre; Bel: Belgium; Bra: Brazil; Bul: Bulgaria; Cam: Cameroon; Can: Canada; CC: Clonal complex; Chi: Chile; Col: Colombia; Cro: Croatia; Cyp: Cyprus; Cze: Czech Republic; Den: Denmark; Det: Detmold; Egy: Egypt; Es: Estonia; Fin: Finland; Fr: Franc; Ga: Gabon; Ger: Germany; Gr: Greece; HK: Hong Kong; Hun: Hungary; Ice: Iceland; In: India; Ire: Ireland; Isr: Israel; It: Italy; Jap: Japan; Kor: Korea; Ku: Kuwait; La: Lativa; Ma: Macedonia; Mal: Malaysia; Mart: Martinique; Mex: Mexico; Min: Minden; MLST: Multilocus sequence; Moz: Mozambique; Net: Netherland; Nor: Norway; NZ: New Zealand; Par: Paraguay; Pol: Poland; Por: Portugal; Ro: Romania; SA: South Africa; Sca: Scandinavia; Sin: Singapore; Slo: Slovenia; Spa: s. aureus protein A; Sp: Spain; ST: Sequence type; Swe: Sweden; Swit: Switzerland; Tai: Taiwan; Tur: Turkey; Ug: Uganda; UK: United Kingdom; Uru: Uruguay; USA: United States of America

${ }^{A}$ Widely disseminated in Europe and Middle East, accounts for considerable percent of isolates in Greece, ${ }^{\mathrm{B}}$ When present, it tends to be abundant; accounts for 54\% isolates in Por, $66 \%$ in Malta, $80 \%$ in Ire and Azores; This clone is also increasingly detected in UK; This clone is $[P V L-]$, $[T s t 1-]$ but a $[P V L+]$ variant and a $[T s t 1+]$ variant exist; the $[T s t 1+]$ variant occurs in some parts of the Middle East; the $[P V L+]$ variant occurs in some European countries, Middle east, and Aust, ${ }^{\mathrm{C}}$ spread extensively across USA.

Alternatively, MRSA can be classified by epidemiological criteria. The epidemiologic definition of CA-MRSA is any strain isolated in an outpatient setting or within 48 hours of admission in a patient with no prior history of MRSA infection or colonization, no permanent invasive medical devices, and none of the following risk factors within the past year: hemodialysis, surgery, hospitalization, residence in a long-term care facility. ${ }^{1,11}$ Strains not fitting this definition are considered HA-MRSA. Nonetheless, we now know that both genotypes can be acquired regardless of risk factors. ${ }^{1}$ Therefore, the better practice would be to use epidemiological criteria to label isolates as community- onset (CO-MRSA) versus hospital-onset (HO-MRSA).

For the sake of our review, we use the genetic makeup to identify CA-MRSA or HA-MRSA and, when provided, the epidemiological criteria to distinguish COMRSA from HO-MRSA.

Antimicrobial Susceptibilities: We reviewed reported susceptibility rates against rifampin, fusidic acid, clindamycin, trimethoprim-sulfamethoxazole (TMPSMX), ciprofloxacin, tetracycline, erythromycin, vancomycin, linezolid, teicoplanin, daptomycin, mupirocin, and gentamicin. 
Methodology. We used the PubMed search engine to identify articles published from January 2005 to June 2019. We selected 13 out of the 18 Arab countries listed in section the Middle East and North African Region, geographically spread across the MENA region. The area was subdivided into the Gulf Cooperation Council (GCC), which includes KSA, Kuwait, UAE, Qatar, Bahrain and Oman; the Levant region, which includes Lebanon, Palestine, Jordan, and Iraq; and the North African region, which includes Egypt, Algeria, and Tunisia. For our search we used the following terms: "Methicillin-Resistant Staphylococcus aureus", "Saudi Arabia", "Kuwait", "United Arab Emirates", "Oman", "Qatar", "Bahrain", "Iraq", "Lebanon", "Palestine", "Gaza strip", "West bank", "Jordan", "Syria", "Egypt", "Algeria," "Tunisia." Our search retrieved 134 articles. We excluded articles that lacked clear methodology and articles written in languages other than Arabic and English. We also disregarded articles that were not accessible unless all relevant information was clearly provided in the abstract. Finally, we included 20 articles from KSA, 8 from Kuwait, 5 from UAE, 2 from Qatar, 1 from Bahrain, 7 from Lebanon, 5 from Jordan, 3 from Gaza, 1 from West Bank, 10 from Algeria, 5 from Tunisia, and 7 from Egypt.

\section{Results.}

MRSA colonization in the MENA region:

MRSA Nasal Carriage: Subjects with MRSA isolated from nasal/pharyngeal swabs without symptoms attributed to MRSA infection are considered colonized. We report MRSA carriage rate as a percent of total nasal/pharyngeal swabs growing MRSA (i.e., total MRSA/total swabs). Figure 1 provides the MRSA carriage rate of total subjects combined from all studies per country. Table 2 lists rates stratified according to four cohorts (outpatients, inpatients, healthy volunteers, and HCWs).

When combining all subjects reported per country, the colonization rates ranged from $2 \%-16 \%$ in GCC, ${ }^{12-22}$ $1-9 \%$ in Levant, ${ }^{23-30}$ and $0.2 \%-9 \%$ in North African Arab states. ${ }^{12,31-36}$ In the GCC, reported rates were overall higher in KSA and Oman compared to Kuwait. In the Levant and North Africa, Lebanon and Tunisia had very low rates.

Several factors may account for the difference in reported rates between studies. These include the difference in study cohorts (i.e., high risk vs. low risk for colonization), the difference in the study period, and the difference in local factors of the study site (i.e., resourcelimited vs. resource-rich city or center). It is

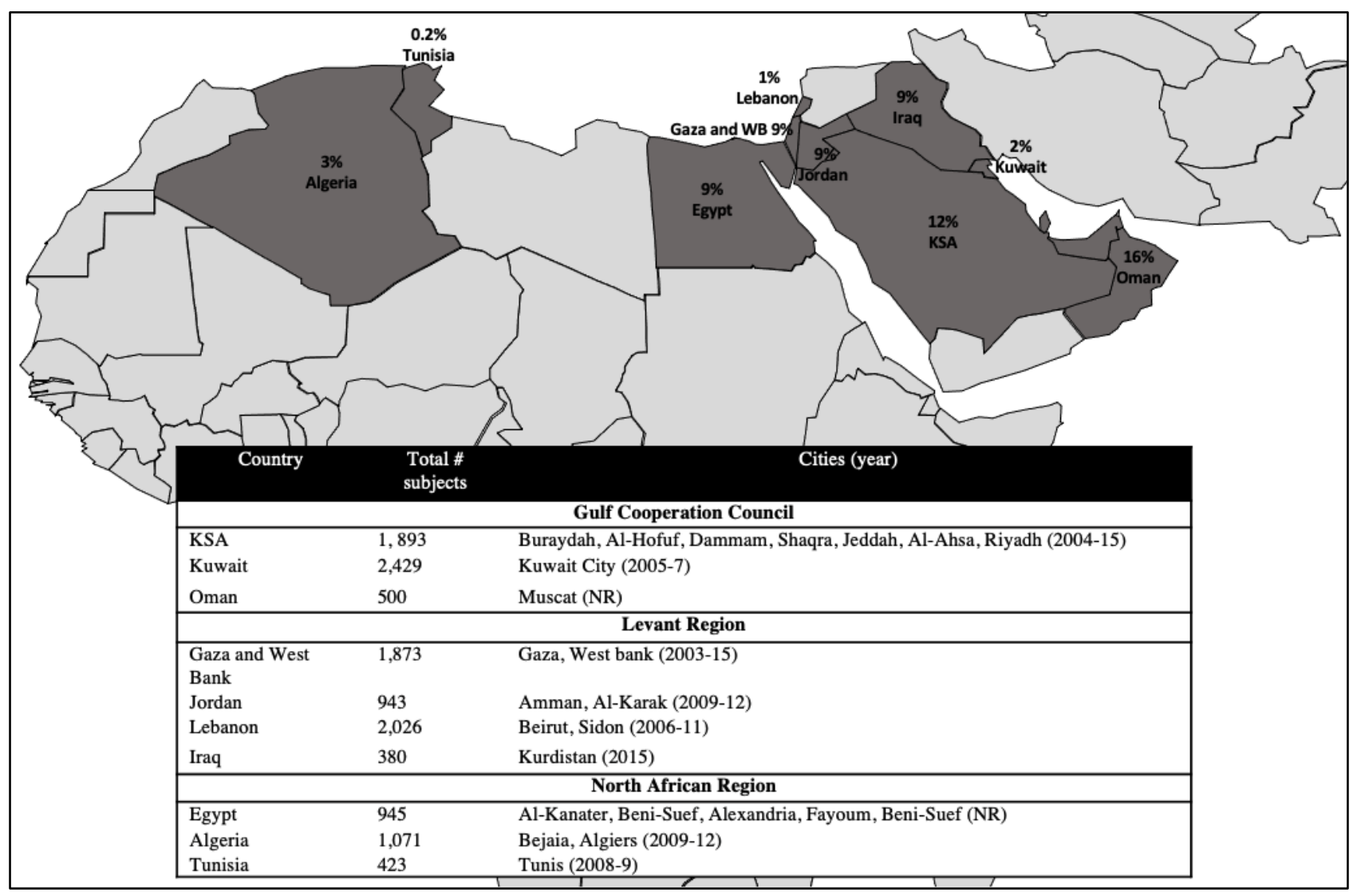

Figure 1. Reported Rates of MRSA Colonization in the MENA Region. MRSA carriage rate reported as \% of total nasal/pharyngeal swabs growing MRSA (i.e., total MRSA/total swabs). The reported rates in the figure are for the total number of subjects from all cited studies per country.

KSA [12-19]; Kuwait [20]; Oman [21, 22]; Gaza and West Bank [23-25]; Jordan [26, 27]; Lebanon [28, 29]; Iraq [30]; Egypt [12, 31-33]; Algeria [34, 35]; Tunisia [36]. 
Table 2. Reported Rates of MRSA Colonization in the MENA Region Stratified based on Cohorts.

\begin{tabular}{|c|c|c|c|c|c|}
\hline Ref & $\begin{array}{c}\text { Total \# } \\
\text { subjects }\end{array}$ & $\%$ Subjects with risk factors for MRSA & \# MRSA(\%) & City (year) & Site \\
\hline \multicolumn{6}{|c|}{ KSA [12-19] } \\
\hline \multirow[t]{2}{*}{ [12] } & 103 OP & $0 \%$ & $26(25 \%)$ & $\begin{array}{l}\text { Buraydah } \\
(2011)\end{array}$ & Multiple hospitals ( $\mathrm{n}=\mathrm{NS}$ ) \\
\hline & $1,048 \mathrm{IP}^{\mathrm{A}}$ & $31 \%$ & $76(7 \%)$ & & \\
\hline$[13]$ & 600 & & $7(1 \%)$ & $\begin{array}{l}\text { Al-Hofuf } \\
(2004)\end{array}$ & King Fahad Hospital \\
\hline$[18]$ & $228^{\mathrm{B}}$ & & $21(9 \%)$ & $\begin{array}{l}\text { Dammam } \\
(2011)\end{array}$ & King Fahad Specialist Hospital \\
\hline \multirow[t]{2}{*}{ [14] } & 220 & & $48(22 \%)$ & $\begin{array}{l}\text { Shaqra (2014- } \\
15)\end{array}$ & Shaqra General Hospital \\
\hline & $152 \mathrm{CV}$ & $\mathbf{0 \%}$ & $1(1 \%)$ & & \\
\hline [15] & $\begin{array}{ll} & 50 \\
\end{array}$ & & $0(0 \%)$ & Jeddah (NR) & King Abdulaziz University \\
\hline [16] & 70 & & $1(1 \%)$ & Al-Ahsa (NR) & NS \\
\hline \multirow[t]{2}{*}{ [17] } & $32^{\mathrm{C}}$ & & $0(0 \%)$ & $\begin{array}{l}\text { Jeddah (2014- } \\
15)\end{array}$ & King Abdulaziz University \\
\hline & $590 \mathrm{HCWs}$ & $100 \%$ & $128(22 \%)$ & & \\
\hline [19] & 200 & & $36(18 \%)$ & $\begin{array}{l}\text { Riyadh (2012- } \\
13) \\
\end{array}$ & $\begin{array}{l}\text { King Khaled University } \\
\text { Hospital }\end{array}$ \\
\hline [15] & 100 & & $73(73 \%)$ & Jeddah (NR) & Multiple hospitals ( $\mathrm{n}=\mathrm{NS}$ ) \\
\hline [16] & $68^{\mathrm{D}}$ & & $3(4 \%)$ & Al-Ahsa (NR) & Multiple hospitals $(\mathrm{n}=6)$ \\
\hline [16] & 72 & & $6(8 \%)$ & Al-Ahsa (NR) & Multiple hospitals $(\mathrm{n}=6)$ \\
\hline [17] & $150^{\mathrm{D}}$ & & $10(7 \%)$ & $\begin{array}{l}\text { Jeddah }(2014- \\
15)\end{array}$ & King Abdulaziz University \\
\hline \multicolumn{6}{|c|}{ Kuwait [20] } \\
\hline [20] & $2,429 \mathrm{IP}^{\mathrm{E}}$ & $100 \%$ & $42(2 \%)$ & $\begin{array}{l}\text { Kuwait City } \\
(2005-7)\end{array}$ & Farwania Hospital \\
\hline \multicolumn{6}{|c|}{ Oman $[21,22]$} \\
\hline & $312 \mathrm{CV}$ & $2 \%$ & $48(15 \%)$ & & \\
\hline [21] & 189 & & $34(18 \%)$ & Muscat (NR) & $\begin{array}{l}\text { Oman Medical College and } \\
\text { Sultan Qaboos University } \\
\text { Hospital }\end{array}$ \\
\hline \multirow[t]{2}{*}{ [22] } & $\cdot 123^{\mathrm{C}}$ & & $14(11 \%)$ & Muscat (NR) & Oman Medical College \\
\hline & $188 \mathrm{HCWs}$ & $100 \%$ & $31(17 \%)$ & & \\
\hline [21] & 116 & & $16(14 \%)$ & Muscat (NR) & $\begin{array}{l}\text { Oman Medical College and } \\
\text { Sultan Qaboos University } \\
\text { Hospital }\end{array}$ \\
\hline [22] & $72^{\mathrm{D}}$ & & $15(21 \%)$ & Muscat (NR) & Oman Medical College \\
\hline \multicolumn{6}{|c|}{ Gaza and West Bank [23-25] } \\
\hline$[24]$ & $843 \mathrm{IP}^{\mathrm{A}}$ & $0 \%$ & $17(2 \%)$ & \begin{tabular}{|l|} 
West bank \\
$(2003)$
\end{tabular} & Ramallah Government hospital \\
\hline \multirow[t]{2}{*}{ [23] } & $758 \mathrm{CV}$ & $3 \%$ & $94(12 \%)$ & Gaza (2009) & $\begin{array}{l}\text { Multiple Gaza neighborhoods } \\
(\mathrm{n}=12)\end{array}$ \\
\hline & $272 \mathrm{HCWs}$ & $100 \%$ & $61(22 \%)$ & & \\
\hline [25] & $\begin{array}{ll}\cdot & 200 \\
\end{array}$ & & $51(26 \%)$ & Gaza (2015) & Al Shifa Hospital \\
\hline [24] & 72 & & $10(14 \%)$ & $\begin{array}{l}\text { West Bank } \\
(2003)\end{array}$ & Ramallah Government hospital \\
\hline \multicolumn{6}{|c|}{ Jordan [26, 27] } \\
\hline & $646 \mathrm{CV}$ & $4 \%$ & $43(7 \%)$ & & \\
\hline [26] & . $\quad 227$ & & $17(8 \%)$ & Amman (2009) & University of Jordan \\
\hline [27] & 419 & & $26(6 \%)$ & $\begin{array}{l}\text { Alkarak (2011- } \\
\text { 12) }\end{array}$ & Al-Karak Hospital \\
\hline$[27]$ & 297 HCWs & $100 \%$ & $30(10 \%)$ & $\begin{array}{l}\text { Al-Karak } \\
(2011-12)\end{array}$ & Al-Karak Hospital \\
\hline \multicolumn{6}{|c|}{ Lebanon $[28,29]$} \\
\hline$[28]$ & 1,526 OP & $17 \%$ & $2(0.1 \%)$ & $\begin{array}{l}\text { Beirut (2010- } \\
11)\end{array}$ & Rizk Hospital, Beirut \\
\hline [29] & $500 \mathrm{CV}^{\mathrm{C}}$ & $12 \%$ & $8(2 \%)$ & $\begin{array}{l}\text { Beirut, Sidon } \\
(2006-7)\end{array}$ & NS \\
\hline \multicolumn{6}{|c|}{ Iraq [30] } \\
\hline$[30]$ & $198 \mathrm{CV}$ & $0 \%$ & $8(4 \%)$ & $\begin{array}{l}\text { Kurdistan } \\
(2015)\end{array}$ & NS \\
\hline
\end{tabular}




\begin{tabular}{|c|c|c|c|c|c|}
\hline [30] & $182 \mathrm{HCWs}$ & $100 \%$ & $25(14 \%)$ & $\begin{array}{l}\text { Kurdistan } \\
\text { (2015) }\end{array}$ & NS \\
\hline \multicolumn{6}{|c|}{ Egypt $[12,31-33]$} \\
\hline & $130 \mathrm{OP}^{\mathrm{F}}$ & $0 \%$ & $36(28 \%)$ & & \\
\hline [31] & 27 & & $3(11 \%)$ & $\begin{array}{l}\text { Beni-Suef } \\
\text { (NR) }\end{array}$ & Beni-Suef University \\
\hline [12] & 103 & & $33(32 \%)$ & $\begin{array}{l}\text { Al Kheireya } \\
\text { (2011) }\end{array}$ & - \\
\hline [32] & $360 \mathrm{IP}^{\mathrm{G}}$ & NS & $29(8 \%)$ & $\begin{array}{l}\text { Alexandria } \\
\text { (NR) }\end{array}$ & Alexandria University \\
\hline \multirow[t]{2}{*}{ [32] } & $470 \mathrm{CV}^{\mathrm{G}}$ & NS & $32(7 \%)$ & $\begin{array}{l}\text { Alexandria } \\
\text { (NR) }\end{array}$ & Alexandria University \\
\hline & 475 HCWs & $100 \%$ & $56(12 \%)$ & & \\
\hline [33] & $\begin{array}{ll}\cdot \quad 223 \\
\end{array}$ & & $30(14 \%)$ & Fayoum (NR) & Fayoum University Hospital \\
\hline [32] & 191 & & $21(11 \%)$ & $\begin{array}{l}\text { Alexandria } \\
\text { (NR) }\end{array}$ & Alexandria University \\
\hline [31] & 61 & & $5(8 \%)$ & $\begin{array}{l}\text { Beni-Suef } \\
\text { (NR) }\end{array}$ & Beni-Suef University \\
\hline \multicolumn{6}{|c|}{ Algeria $[34,35]$} \\
\hline [34] & 612 IP & $33 \%$ & $9(2 \%)$ & $\begin{array}{l}\text { Bejaia (2010- } \\
12)\end{array}$ & $\begin{array}{l}\text { Frantz-fanon nephrology } \\
\text { department and Amizour } \\
\text { Hospital }\end{array}$ \\
\hline [35] & $459 \mathrm{CV}^{\mathrm{G}}$ & NS & $23(5 \%)$ & $\begin{array}{l}\text { Algiers (2009- } \\
11)\end{array}$ & $\begin{array}{l}\text { Centre Hospitalo-Universitaire } \\
\text { Mustapha Bacha }\end{array}$ \\
\hline \multicolumn{6}{|c|}{ Tunisia [36] } \\
\hline [36] & $423 \mathrm{CV}$ & $23 \%$ & $1(0.2 \%)$ & Tunis (2008-9) & Université Tunis El Manar \\
\hline
\end{tabular}

MRSA carriage rate reported as \% of total nasal/pharyngeal swabs growing MRSA (i.e., total MRSA/total swabs). Bolded rows represent entire cohort. Risk factors for MRSA: isolate recovered $>48$ hours of hospitalization, invasive medical devices, healthcare workers (including medical/pharmaceutical students with clinical exposure) and the following within the preceding year: hemodialysis, surgery, hospitalization, residence in a long-term care facility.

CV: community volunteer(s); HCWs: healthcare worker(s); IP: inpatient(s); NS: not specified; OP: outpatient(s). ${ }^{\mathbf{A}}$ Screened $<48$ hours of admission, ${ }^{\mathbf{B}}$ Cancer patients, ${ }^{\mathbf{C}}$ Students without clinical exposure (considered community volunteers), ${ }^{\mathbf{D}}$ Students with clinical exposure (considered HCWs), ${ }^{\mathbf{E}}$ ICU patients [screened on admission and weekly thereafter; hence, all considered at risk for MRSA colonization], ${ }^{\mathbf{F}} 27$ subjects were dental clinic outpatients whose risk factors were not specified, ${ }^{\mathbf{G}}$ access to abstract only.

difficult through our review to determine which factors explain the observed difference in rates. The cited studies from Lebanon were limited to outpatients and community volunteers only as opposed to Gaza, Jordan, and Iraq, where some studies included HCWs. Nonetheless, even when comparing similar groups, the rates in Lebanon remained lower. This is true for Beirut and Sidon, 2 heavily populated cities in Lebanon. ${ }^{28,29} \mathrm{It}$ is also unclear why Kuwait in GCC and Tunisia in North Africa had lower rates than neighboring states. The study from the city of Kuwait included 2,429 intensive care patients screened on admission and weekly thereafter, yet only $2 \%$ had MRSA colonization. ${ }^{20}$ In Tunisia, only $0.2 \%$ of 423 community volunteers carried MRSA, ${ }^{36}$ while rates in similar groups reached 5\% in Algeria and $7 \%$ in Egypt. ${ }^{12,31-35}$

Moreover, the reported rates of MRSA carriage varied widely between centers and communities within the same country. Here again, the difference in rates may be driven by the aforementioned factors. Perhaps the interplay between varying environmental and social factors across a county could explain why certain areas serve as pockets for higher prevalence. In the healthcare setting, discrepancies in rates between hospitals might be driven by differences in the type of patients served i.e., high acquity versus low acuity hospitals) and the institutional infection control efforts.

\section{MRSA Infections in the MENA Region:}

MRSA Infection Rates: We report rates of MRSA infection as percent of clinical $S$. aureus isolates showing methicillin resistance (total MRSA/total $S$. aureus derived from clinical specimen). Figure 2 demonstrates percent methicillin resistance in total isolates derived from all studies per country. Supplementary table 2 provides more details for each cited study.

When examining all isolates recovered per country, methicillin-resistance ranged from 9\%-38\% in GCC, ${ }^{11,37-}$ ${ }^{44} 28 \%-67 \%$ in the Levant, ${ }^{45-53}$ and $28 \%-57 \%$ in North African states. ${ }^{54-65}$ In the GCC, lower rates were recorded in UAE and Qatar compared to Oman and KSA. In UAE, the highest rate reached $29 \%$ in a study from Dubai examining $62 \mathrm{~S}$. aureus isolates derived from purulent skin and soft tissue infections (SSTI). ${ }^{41}$ In the Levant and North Africa, Lebanon and Tunisia again had the lowest rates in clinical isolates. Notably, one of the studies from Lebanon saw a rate of $72 \%$ resistance in 32 isolates. $^{48}$ These isolates were randomly selected, not consecutive, and examined only wound and respiratory specimen. A subsequent study testing 4,890 isolates from 16 different hospitals across Lebanon showed a much lower rate of $28 \%{ }^{49}$ In Iraq, very high rates were seen in combat 


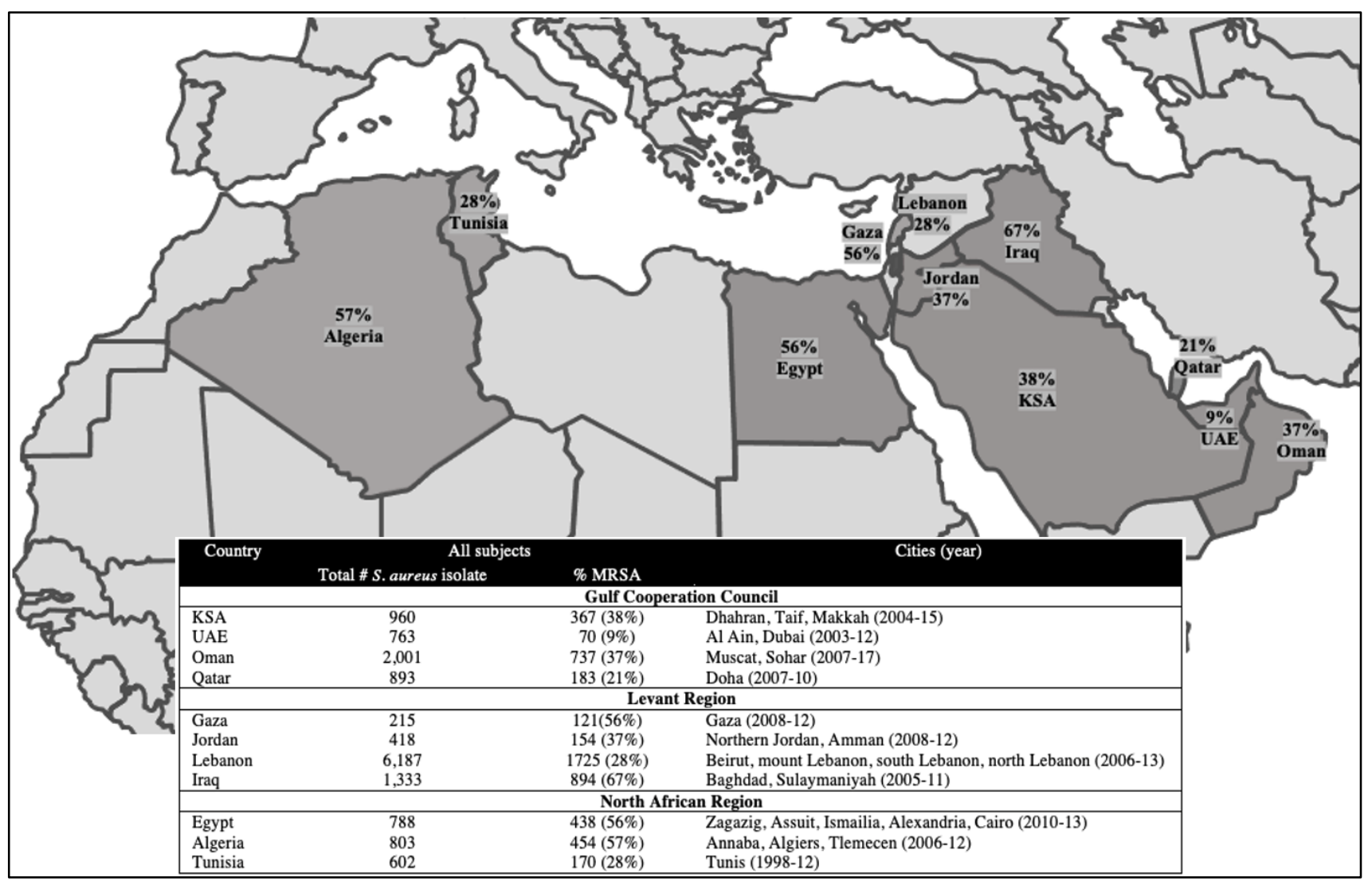

Figure 2. Reported Rates of Methicillin-resistance among Staphylococcus aureus from Clinical Specimen in the MENA Region. The figure demonstrates rates as percent of all clinical $S$. aureus isolates derived from all studies per country showing methicillin resistance (total MRSA/total $S$. aureus derived from clinical specimen).

KSA [37-40] UAE [41] Oman [42, 43] Qatar [11, 44] Gaza[45] Jordan [46,47] Lebanon [48-50] Iraq [51-53] Egypt [54-57] Algeria[58-62] Tunisia [63-65]

support hospitals among U.S. military personnel, ${ }^{51,52}$ particularly when examining skin and soft tissue infections (SSTI), and in a burn center where the rate reached $88 \% .{ }^{53}$ In North Africa, Algeria reached a high of $86 \%$ in Annaba when examining diabetic foot infections. ${ }^{60} \mathrm{~A}$ high rate of $75 \%$ in another study was also seen in surgical wound infections $>48$ hours postoperatively in Tlemecen. ${ }^{62}$ On the other hand, a study from Algiers showed a lower rate of $19 \%$ which again highlights the varying epidemiology depending on site within a country. ${ }^{59}$ When testing clinical isolates from neutropenic patients in Tunisia, a rate of $18 \%$ was detected, still lower than most studies in Egypt and Algeria. ${ }^{63}$

Only few studies specified site of acquisition based on epidemiological criteria, i.e., CO-MRSA vs HOMRSA, outlined in table 3. Based on these few studies, isolates causing hospital-onset infections showed higher rates of methicillin resistance. S. aureus isolates causing community-onset infections had 51\% methicillin resistance in $\mathrm{KSA}^{40}, 12 \%-47 \%$ in Egypt, ${ }^{56,57} 24 \%-40 \%$ in Algeria, ${ }^{58,59,61,62} 29 \%$ in Tunisia. ${ }^{64}$ In Egypt, the $40 \%$ rate was in community-onset SSTI. ${ }^{56}$ On the other hand, isolates causing hospital-onset infection had $66 \%$ resistance in $\mathrm{KSA}^{40}{ }^{40} 77 \%$ in Egypt ${ }^{56,57} 47 \%-75 \%$ in Algeria, ${ }^{58,59,61,62} 18 \%-55 \%$ in Tunisia. ${ }^{63,64}$
Molecular Types of MRSA in MENA Region: As expected, heterogeneity between studies in the typing techniques utilized and the reporting of results was evident. Some studies reported SCCmec types alone while others reported MLST and/or spa typing with or without CC assignment. Therefore, determining the relatedness of reported strains from different studies is challenging. For example, in the study from Bahrain, PFGE typing was used. While this is a good tool to investigate relatedness of strains in an institution, it does not allow for comparison with other centers. ${ }^{6}$

Studies demonstrated a wide clonal diversity across the region. This was evident by the large number of different spa and/or MLST types and their corresponding $\mathrm{CCs}$. Furthermore, the epidemiology varied depending on center, city, study period, and subject cohort.

The bulk of studies recovered in our review did not classify their samples based on site of acquisition (COMRSA vs HO-MRSA). This would have been a valuable tool to distinguish the epidemiology between community and healthcare settings.

All studies examining colonizing MRSA were limited by a small sample size. Table 4 lists the relative frequencies of $P V L, T s t 1$, SCCmes, MLST, Spa, and CCs in colonizing strains. Supplementary table 3 organizes 
Table 3. Stratification of clinical isolates based on epidemiological criteria.

\begin{tabular}{|l|c|c|c|c|c|c|c|}
\hline \multirow{2}{*}{ Ref } & \multicolumn{3}{|c|}{ S. aureus isolates } & \multicolumn{3}{c|}{ MRSA isolates } & \multirow{2}{*}{} \\
\cline { 2 - 7 } & Total \# & CO & HO & Total \# & CO & HO & \\
\hline$[5]$ & $\mathbf{2 5 2}^{\mathbf{A}}$ & 136 & 116 & $\mathbf{1 4 6}$ & 70 & 76 & KSA \\
\hline$[7]$ & $\mathbf{6 2}^{\mathbf{B}}$ & NS & NS & $\mathbf{1 8}$ & 17 & 1 & UAE \\
\hline$[23]$ & $\mathbf{3 8}^{\mathbf{C}, \mathbf{D}}$ & 38 & 0 & $\mathbf{1 8}$ & 18 & 0 & Egypt \\
\hline$[24]$ & $\mathbf{6 3 1}^{\mathbf{A}}$ & 183 & 448 & $\mathbf{3 6 4}$ & 21 & 343 & Egypt \\
\hline$[25]$ & $\mathbf{1 4 8}^{\mathbf{A}}$ & 29 & 119 & $\mathbf{9 2}$ & 7 & 85 & Algeria \\
\hline$[26]$ & $\mathbf{1 2 9}^{\mathbf{A}}$ & NS & NS & $\mathbf{2 5}$ & 10 & 15 & Algeria \\
\hline$[28]$ & $\mathbf{2 2 1}^{\mathbf{A}}$ & 84 & 137 & $\mathbf{9 9}$ & 34 & 65 & Algeria \\
\hline$[29]$ & $\mathbf{2 2 0}^{\mathbf{C}, \mathbf{E}}$ & 0 & 220 & $\mathbf{1 6 5}$ & 0 & 165 & Algeria \\
\hline$[30]$ & $\mathbf{7 2}^{\mathbf{F}}$ & 0 & 72 & $\mathbf{1 3}$ & 0 & 13 & Tunisia \\
\hline$[31]$ & $\mathbf{1 4 3}^{\mathbf{A}}$ & 77 & 66 & $\mathbf{5 8}$ & 22 & 36 & Tunisia \\
\hline
\end{tabular}

CO: community-onset; HO: hospital-onset; NS: not specified. A Various clinical infections, ${ }^{\text {B }} \underline{\text { Purulent }}$ SSTI, ${ }^{\text {C }}$ SSTI, D Study examined community-onset infections only, ${ }^{\mathrm{E}}$ Study examined hospital-onset infections only, ${ }^{\mathrm{F}}$ Neutropenic patients in bone marrow transplant center.

the genotypes into clones. In the GCC, $P V L$ gene carriage reached $12 \%$ in outpatients in KSA. ${ }^{12} T_{s} t 1$ gene prevalence was not examined in studies from GCC. ${ }^{12,16,20}$ Low $P V L$ carriage was also detected in the Levant, $3 \%$ $9 \%{ }^{23,26,27} T_{s t 1}$ was examined in Jordan only, and very high rates were depicted in both community and healthcare settings. ${ }^{26,27}$ In North Africa, $P V L$ carriage was low in Egypt, $2 \%-15 \%,{ }^{12,31,33}$ but reached $33 \%$ in Algeria, ${ }^{34}$ while Tst 1 carriage reached $22 \%$ in Algeria, ${ }^{34}$ but $50 \%$ in Egypt. ${ }^{31}$

Table 4. Molecular Typing of Colonizing MRSA Isolates in the MENA Region.

\begin{tabular}{|c|c|c|c|c|c|c|c|c|}
\hline Ref & $\begin{array}{c}\# \\
\text { MRSA }\end{array}$ & $P V L$ & Tst1 & SCCmec (\%) & $\operatorname{Spa}(\%)$ & MLST & $\begin{array}{c}\text { Clonal } \\
\text { Complexes/PFGE/singletons } \\
(\%)\end{array}$ & $\begin{array}{l}\text { Study } \\
\text { location } \\
\text { (year) }\end{array}$ \\
\hline \multicolumn{9}{|c|}{ KSA } \\
\hline [12] & $26 \mathrm{OP}$ & $12 \%$ & ND & $\begin{array}{c}\text { V (42), NT } \\
(35), \text { IVa (23) }\end{array}$ & $\begin{array}{c}\mathbf{t 0 8 4}(14), \text { t085 (13), t008 (13), t349 } \\
\text { (7), t660 (7), t002 (7), t223 (7), } \\
\text { t376 (7), t189 (7), t442 (7), t6836 } \\
(7), \text { t11841 (7) }\end{array}$ & ND & $\begin{array}{c}\mathrm{CC} 15 \text { (27), CC8 (13), CC25 } \\
\text { (13), CC5 (7), CC22 (7), } \\
\text { CC80 (7), CC188 (7), CC-NA } \\
(20)\end{array}$ & $\begin{array}{l}\text { Buraydah } \\
(2011)\end{array}$ \\
\hline [16] & $20 \mathrm{CV}$ & ND & ND & $\begin{array}{l}\text { IV (90), IVa } \\
\quad(10)\end{array}$ & $\begin{array}{l}\text { t131 (20), t1328 (20), t688 (20), } \\
\text { t304 (20), t128(10), t1339 (10) }\end{array}$ & ND & $\begin{array}{c}\text { CC80 (20), CC22 (20), CC5 } \\
(20), \text { PFGE F (20), CC1 (10), } \\
\text { PFGE K CC88 (10) }\end{array}$ & $\begin{array}{l}\text { Al-Ahsa } \\
\text { (NR) }\end{array}$ \\
\hline \multicolumn{9}{|c|}{ Kuwait } \\
\hline [20] & $18 \mathrm{IP}^{\mathrm{B}}$ & ND & ND & $\begin{array}{l}\text { III (72), IV } \\
\quad(28)\end{array}$ & $\begin{array}{c}\text { t421 (33), t945 (28), t4410 (6), t388 } \\
(6), \mathbf{t 2 2 3}(6), \mathrm{t} 3010(6), \mathrm{t} 6665(6), \\
\text { t1234 (6), t044 (6) }\end{array}$ & $\begin{array}{l}\text { ST239 (72), ST22 } \\
(11), \text { ST97 (11), } \\
\text { ST80 (6) } \\
\end{array}$ & ND & $\begin{array}{l}\text { Kuwait } \\
\text { City } \\
(2005-7)\end{array}$ \\
\hline \multicolumn{9}{|c|}{ Gaza } \\
\hline [23] & $94 \mathrm{CV}$ & $9 \%$ & ND & $\begin{array}{l}\text { IV (1), IVa } \\
(81), \mathrm{V}(11) \\
\text { ND (5), IVc } \\
\quad(2)\end{array}$ & $\mathbf{t} 223(76)^{\mathbf{A}}$ & $\begin{array}{c}\text { ST22 (74), ST78 } \\
(7), \text { ST80 (5), ST- } \\
\text { ND (5), ST1784 } \\
(2), \text { ST1785 (2), } \\
\text { ST5 (1), ST30 (1), } \\
\text { ST1734 (1), } \\
\text { ST913 (10) }\end{array}$ & $\begin{array}{c}\mathbf{C C 2 2}(76), \operatorname{CC} 88 \text { (7), CC80 } \\
\text { (5), CC5 (3), CC30 (2), } \\
\text { CC913 (1), CC-ND (5) }\end{array}$ & $\begin{array}{l}\text { Gaza } \\
(2009)\end{array}$ \\
\hline \multicolumn{9}{|c|}{$\begin{array}{ll} & \text { Jordan } \\
\end{array}$} \\
\hline [26] & $\begin{array}{c}37 \\
\mathrm{HCW} \\
\end{array}$ & $5 \%$ & $100 \%$ & $\begin{array}{l}\text { IVe (81), IV } \\
(14), \text { IVc (5) }\end{array}$ & $\begin{array}{c}\text { t9519 (73), t223 (14), t044 (5), NT } \\
(8)\end{array}$ & ND & ND & $\begin{array}{c}\text { Amman } \\
(2009)\end{array}$ \\
\hline \multirow[t]{2}{*}{ [27] } & $26 \mathrm{CV}$ & $8 \%$ & $58 \%$ & $\begin{array}{l}\text { IVa (69), IVc } \\
(19), \text { NT (8), } \\
\text { IVg (4) }\end{array}$ & $\begin{array}{c}\text { t223 (39), t214 (12), t021 (8), t1651 } \\
(8), \text { t386 (8), t6397 (4), t790 (4), } \\
\text { t012 (4), t253 (4), t044 (4), t1234 } \\
(4), \text { t803 (4) }\end{array}$ & ND & $\begin{array}{c}\mathbf{C C 2 2}(46), \mathbf{C C 3 0}(23), \mathbf{C C 5} \\
(12), \text { CC1 (8), CC80 (4), } \\
\text { CC97 (4), CC15 (4) }\end{array}$ & \multirow[t]{2}{*}{$\begin{array}{l}\text { Al-karak } \\
(2011-12)\end{array}$} \\
\hline & $\begin{array}{c}30 \\
\mathrm{HCW}\end{array}$ & $3 \%$ & $57 \%$ & $\begin{array}{l}\text { IVa }(80), \text { IVc } \\
(17), \mathrm{V}(3)\end{array}$ & $\begin{array}{l}\text { t223 (40), t386 (20), t309 (7), t018 } \\
(7), \text { t10683 (7), t021 (6), t693 (3), } \\
\text { t012 (3), t044 (3), t370 (3) }\end{array}$ & ND & $\begin{array}{c}\text { CC22 (47), CC1 (23), CC30 } \\
(17), \text { CC5 (7), CC80 (4), } \\
\text { CC45 (3) }\end{array}$ & \\
\hline
\end{tabular}




\begin{tabular}{|c|c|c|c|c|c|c|c|c|}
\hline \multicolumn{9}{|c|}{ Egypt } \\
\hline [33] & $\begin{array}{c}30 \\
\mathrm{HCW} \\
\end{array}$ & $2 \%$ & ND & ND & ND & ND & ND & $\begin{array}{c}\text { Fayoum } \\
\text { (NR) }\end{array}$ \\
\hline [31] & $21 \mathrm{OP}$ & $15 \%$ & $50 \%$ & $\begin{array}{l}\text { IV (48), I } \\
(38), \text { V (5), } \\
\text { NT (5), II (5) }\end{array}$ & $\begin{array}{c}\text { t223 (24), t267 (24), t14339(20), } \\
\text { t3689 (10), t084 (10), t380(10), } \\
\text { t8506 (5), t1339(5) }\end{array}$ & ND & $\begin{array}{l}\text { Spa-CC223 (52), Spa-S-t267 } \\
(24), S p a-\text { S-t084 (10), Spa- S- } \\
\text { t380 (10), Spa-S-t1339 (5) }\end{array}$ & $\begin{array}{l}\text { Beni-Suef } \\
\text { (NR) }\end{array}$ \\
\hline [12] & $33 \mathrm{OP}$ & $15 \%$ & ND & $\begin{array}{c}\text { IVa (49), } \mathrm{V} \\
\quad(52)\end{array}$ & $\begin{array}{c}\text { t688 (30), t044 (15), t786(15), t021 } \\
(10), \text { t318 (10), t223(10), t008 (5), } \\
\text { t1 } 1839(5) \\
\end{array}$ & ND & $\begin{array}{c}\text { CC5 (30), CC30 (20), CC80 } \\
(15), \text { CC88 (15), CC22 (10), } \\
\text { CC8 (5), CC-NA (5) }\end{array}$ & $\begin{array}{c}\text { Al- } \\
\text { Kanater } \\
(2011)\end{array}$ \\
\hline \multicolumn{9}{|c|}{ Algeria } \\
\hline [34] & 9 IP & $33 \%$ & $22 \%$ & $\begin{array}{l}\text { IVa (33), IVc } \\
\text { (44), IVh } \\
\text { (11), VII } \\
\text { (11) }\end{array}$ & $\mathbf{t} 223(22 \%)^{\mathbf{A}}$ & $\begin{array}{c}\text { ST80 (44\%), } \\
\text { ST22 (22\%), ST5 } \\
(22 \%), \text { ST535 } \\
(11 \%)\end{array}$ & ND & $\begin{array}{c}\text { Bejaia } \\
(2010-12)\end{array}$ \\
\hline
\end{tabular}

Bolded CCs and spa types are frequently reported globally (refer to table $\mathbf{1}$ and supplementary table 1). Values were rounded to the nearest digit. CC: clonal complex; CV: community volunteer; HCW: healthcare worker; IP: inpatient; ND not determined; NT not typeable; OP: outpatient; S-: singleton; Spa-CC: spa clonal complex.

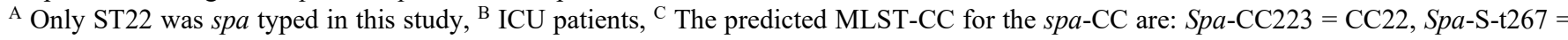
CC80, Spa-S-t084 = CC15, Spa-S-t380 = CC80, Spa-S-t1339 $=$ CC-NT.

Table 5 lists the $P V L$, Tst 1 , SCCmes, MLST, Spa, and CCs in infective strains. Supplementary table 4 organizes the genotypes into clones. In GCC, $P V L$ carriage ranged from $0 \%-77 \%, 7,11,37,66-73$ while $T_{s} t 1$ carriage ranged from $4 \%-24 \% .^{7,66-68,71,73}$ In the Levant, $P V L$ gene was detected in $37 \%-96 \%$ of infective MRSA. ${ }^{45,47,48,74}$ Tst 1 gene was screened for in Gaza and detected in $40 \%$ of isolates. ${ }^{45} \mathrm{PVL}$ detection rate ranged from $21 \%-88 \% .^{54,58,75,76}$ Most cited studies did not determine Tst 1 carriage except one from Algeria where none of the isolates carried the toxin gene. ${ }^{61}$

Both HA-MRSA and CA-MRSA genotypes were frequently detected among colonizing and infective strains in the MENA region. Most of the globally prevalent spa types were detected in the Arab countries, as illustrated in supplementary table 1. Spa types belonging to European CA-MRSA, Brazilian/Hungarian HA-MRSA, and EMRSA-15 HA-MRSA were most widely distributed across the region. The most frequently detected CCs were $\mathrm{CC} 5, \mathrm{CC} 8, \mathrm{CC} 22, \mathrm{CC} 30$, and $\mathrm{CC} 80$, which are globally spread complexes briefly reviewed in table 1. In this section we review the epidemiology of these CCs in the MENA Arab world.

Clonal Complex 5: Members of CC5 are widespread geographically. ${ }^{8}$ Established clones from this group can carry SCCmec-I, II, III, IV, and V and can be HA-MRSA and CA-MRSA. It consists of several clones including the South German Epidemic HA-MRSA (ST228SCCmec-I), Geraldine CA-MRSA (ST5-SCCmec-I), West Australian (W.A.) MRSA-18, and -21 (both ST5SCCmec-I) and -48 (ST835-SCCmec-I), New York/Japan (ST5-SCCmec-II), and Paediatric (ST5SCCmec-IV) clones, among others. ${ }^{8}$ The last 2 clones are described as pandemic. Another clone previously described is ST5-SCCmec-V which is colloquially known as WA MRSA-11, $-14,-34$, and -35 . This isolate was detected in Australia, Ireland, and Germany. ${ }^{8}$

Colonizing isolates of this group were detected in KSA,${ }^{12}$ Gaza ${ }^{23}$ Jordan, ${ }^{27}$ and Egypt. ${ }^{12}$ These included the
CC5-V and CC5-IV which are likely related to WA MRSA and Paediatric clones, respectively. All these strains were [PVL-]. In Egypt, CC5-V made up 30\% of colonizing strains from outpatients. ${ }^{12}$

In infective strains from the MENA, the CC5 and its clones were among the less commonly detected strains. Detected SCCmec types were II, IV, and V. Therefore, observed clones included the Paediatric, New York/Japan, and WA MRSA-(11, -14, -34, and-35).

Clonal Complex 8: Several clones belong to this complex, including both CA-MRSA and HA-MRSA. ${ }^{8}$ Its strains can carry SCCmec-I, IV, V, and VIII. MLST types include ST8, ST72, and ST239/ST240/ST241. It houses the first known MRSA clone (ST250-SCCmec-I), known as Ancestral MRSA. This clone is disappearing with time. It also houses the famous Iberian (ST8SCCmec-I), USA300 CA-MRSA $([P V L+]$ ST8SCCmec-IV+ACME) and Brazilian/Hungarian HAMRSA ([PVL-] ST239-SCCmec-III). USA300 has disseminated throughout the United States of America (U.S.) in few years. Sporadic cases have been identified in some European countries. The Brazilian/Hungarian HA-MRSA is considered the oldest pandemic strain. It is described from countries in every continent. This clone is always $[P V L-]$. It is prevalent in Europe, Australia, Middle East, and Asia and can be divided into 3 clades [European, Asian, and south American]. ST240 and 241 are closely related to ST239, each differ in mutations within 1 housekeeping gene only. ${ }^{8}$

In MENA region, the Brazilian/Hungarian HAMRSA was very prevalent in some centers in Kuwait ${ }^{7,69}$ and $\mathrm{KSA}^{66,67}$ but had a decreasing presence in a center from UAE, ${ }^{71}$ and was completely absent in 1 hospital in Qatar. ${ }^{11}$ It had a modest presence in the Levant. ${ }^{45,47,48,74}$ It was notably nonexistent in combat support hospitals in Iraq ${ }^{74}$ In Algeria, It was very common in Annaba but not in Algiers, ${ }^{58-61,75}$ and in Tunisia, it was frequently encountered in HO-MRSA isolates but not CO-MRSA. ${ }^{75}$ All ST239 isolates in the MENA region were [PVL-] and 
Table 5. Molecular Typing of Infective MRSA Isolates in the MENA Region.

\begin{tabular}{|c|c|c|c|c|c|c|c|c|}
\hline Ref & \#MRSA & $P V L$ & Tst 1 & $\mathrm{SCCmec}(\%)$ & $\operatorname{Spa}(\%)$ & MLST $(\%)$ & Clonal Complexes/PFGE/singletons (\%) & $\begin{array}{l}\text { Study } \\
\text { location } \\
\text { (year) }\end{array}$ \\
\hline \multicolumn{9}{|c|}{$\begin{array}{ll} & \text { KSA } \\
\end{array}$} \\
\hline [66] & $120^{\mathbf{A}}$ & $0 \%$ & $8 \%$ & III (100) & $\begin{array}{c}\text { t037 (35), t044 (19), t030 (5), t363 (5), t304 (4), t002 (4), t631 } \\
(3), \mathrm{t} 690(3), \mathrm{t} 019(2), \mathrm{t} 388(2), \mathrm{t} 032(2), \mathrm{t} 223(2), \mathrm{t} 3059(2), \mathrm{t} 138 \\
(1), \mathrm{t} 459(1), \mathrm{t} 748(1), \mathrm{t} 932(1), \mathrm{t} 1070(1), \mathrm{t} 790(1), \mathrm{t} 4573(1), \\
\mathrm{t} 7604(1), \mathrm{t} 8506(1), \mathrm{t} 8855(1), \mathrm{t} 376(1), \mathrm{t} 8731(1), \mathrm{t} 729(1), \mathrm{t} 8507 \\
(1), \mathrm{t} 701(1), \mathrm{t} 364(1), \mathrm{t} 2235(1)\end{array}$ & ND & $\begin{array}{c}\text { Spa-CC037 (55), spa-CC790 (8), spa-CC376 } \\
\text { (21), cluster } 4 \text { spa CC690 (4), Spa-no } \\
\text { founder (5), S-t002 (4), S-t3059 (2), S-t364 } \\
\text { (1), S-t2235 (1) }\end{array}$ & $\begin{array}{c}\text { Riyadh } \\
(210)\end{array}$ \\
\hline [67] & $107^{\mathbf{B}}$ & $54 \%$ & $8 \%$ & $\begin{array}{l}\text { III (21), IV } \\
(75), \text { V (4) }\end{array}$ & 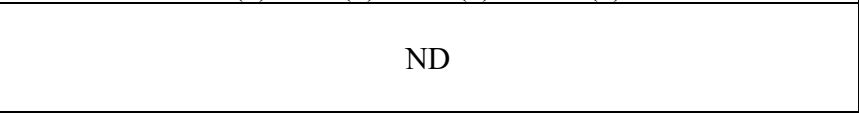 & ST239 (21), ST772 (1), ST834 (1) & $\begin{array}{c}\text { CC8 (21), CC22 (28), CC80 (20), CC30 } \\
(12), \text { CC5 (8), CC6 (3), CC88 (3), CC97 (2), } \\
\text { CC1 (2), CC9 (1), CC45 (1) }\end{array}$ & $\begin{array}{l}\text { Riyadh } \\
\text { (NR) }\end{array}$ \\
\hline [68] & 117 & $49 \%$ & $9 \%$ & $\begin{array}{l}\text { III (8), IV } \\
(80), \text { V (10) }\end{array}$ & ND & ST239 (8), ST8 (1), ST72 (1), ST913C & $\begin{array}{c}\mathbf{C C 8 0}(30), \operatorname{CC6}(13), \operatorname{CC5}(11), \mathbf{C C 2 2}(10), \\
\text { CC8 (9), CC30 (7), CC97 (7), CC88 (5), } \\
\operatorname{CC} 15 \text { (3), CC2250 (2), CC1 (1), CC96 (1), } \\
\text { CC45 (1), CC913 (1) }\end{array}$ & $\begin{array}{l}\text { Riyadh } \\
(2009-15)\end{array}$ \\
\hline [72] & 101 & $38 \%$ & ND & $\begin{array}{l}\text { III (39), IV } \\
\text { (43), IVa } \\
(16), \text { IVc (3) }\end{array}$ & ND & ND & ND & $\begin{array}{c}\text { Jeddah } \\
(2009-11)\end{array}$ \\
\hline [87] & 100 & $19 \%$ & ND & $\begin{array}{l}\text { I (3), II (9), } \\
\text { III (47), IV } \\
(29), \text { NT (12) }\end{array}$ & ND & ND & ND & $\begin{array}{c}\text { Makkah } \\
(2012)\end{array}$ \\
\hline [37] & 71 & $28 \%$ & ND & $\begin{array}{l}\text { I (34), II } \\
(10), \text { III }(20) \\
\text { IV }(20), \text { V } \\
(16), \text { NT (1) }\end{array}$ & ND & ND & ND & $\begin{array}{l}\text { Taif city } \\
(2013-15)\end{array}$ \\
\hline \multicolumn{9}{|c|}{ Kuwait } \\
\hline [69] & $400^{\mathrm{D}}$ & $16 \%$ & ND & $\begin{array}{l}\text { II (5), III } \\
(55), \text { IV (32), } \\
\text { V (8), VI } \\
(0.2)\end{array}$ & $\begin{array}{l}\text { t030, t037, t421, t945, t860, t064, t008, t1902, t138, t459, t032, } \\
\text { t790, t223, t5708, t309, t3935, t2251, t852, t3107, t5983, t002, } \\
\text { t003, t242, t105, t045, t306, t2164, t688, t5258, t1154, t376, } \\
\text { t8154, t1154, t018, t042, t044, t019, t345, t318, t1 130, t605, } \\
\text { t6811, t127, t321, t345, t10795, t657, t12211, t304, t1234, t13204, } \\
\text { t690, t4067, t5041, t5562, t314, t991, t370, t050, t315, t1427, } \\
\text { t4000, t605 }\end{array}$ & $\begin{array}{l}\text { ST239 (46), ST22 (9), ST80 (7), ST5 (7), } \\
\text { ST30 (4), ST241 (3), ST6 (2), ST36 (2), } \\
\text { ST772 (2), ST8 (2), ST97 (2), ST113(1), } \\
\text { ST225 (1), ST105 (1), ST149 (1), ST361 (1), } \\
\text { ST1 (0.3), ST88 (0.3), ST121 (0.3), ST913 } \\
(0.3), \text { ST46 (0.3), ST72 (0.3), ST508 (0.3), } \\
\text { ST1289 }(0.3), \text { ST1465 (0.3), ST1637 (0.3), } \\
\text { ST2816(0.3) }\end{array}$ & $\begin{array}{c}\mathbf{C C 8}(58), \mathbf{C C 2 2}(9), \operatorname{CC5}(9), \operatorname{CC80}(7), \\
\mathbf{C C 3 0}(6), \operatorname{CC} 1(3), \operatorname{CC} 6(2), \operatorname{CC} 97(2), \\
\operatorname{CC} 88(1), \operatorname{CC} 121(1), \operatorname{CC} 913(1), \operatorname{CC} 45(1), \\
\operatorname{CC} 361(1)\end{array}$ & $\begin{array}{l}\text { Multiple } \\
\text { sites } \\
(1992- \\
2010)\end{array}$ \\
\hline [84] & 6922 & ND & ND & $\begin{array}{l}\text { I (0.3), II (1), } \\
\text { III (29), IV } \\
(54), \text { V (15), } \\
\text { NT (1) }\end{array}$ & ND & ND & ND & $\begin{array}{l}\text { Multiple } \\
\text { sites } \\
(2011-15)\end{array}$ \\
\hline [70] & $\begin{array}{l}26 \\
\text { SCCmec- } \\
\text { IV }\end{array}$ & $77 \%$ & ND & $\begin{array}{l}\text { IV (89), IVa } \\
(8), \text { IVc (4) }\end{array}$ & ND & $\begin{array}{c}\text { ST80 (46), ST30 (31), ST5 (8), ST6 (4), } \\
\text { ST8(8), ST361 (4) }\end{array}$ & $\begin{array}{c}\mathbf{C C 8 0}(46), \mathbf{C C 3 0}(31), \mathbf{C C 5}(12), \mathbf{C C 8}(8), \\
\text { CC-ND (4) }\end{array}$ & $\begin{array}{l}\text { Multiple } \\
\text { sites } \\
(2001-3)\end{array}$ \\
\hline
\end{tabular}




\begin{tabular}{|c|c|c|c|c|c|c|c|c|}
\hline [78] & $\begin{array}{c}37 \text { ST22 } \\
\text { Urease } \\
(-)\end{array}$ & $22 \%$ & $62 \%$ & $\begin{array}{l}\text { IV (22), IVa } \\
\text { (70), IVh (8) }\end{array}$ & $\begin{array}{c}\mathbf{t} 223(51), \mathbf{t 0 3 2}(8), \mathrm{t} 853(14), \mathrm{t} 790(8), \mathrm{t} 3107(5), \mathrm{t} 309(3), \mathrm{t} 2251 \\
(3), \mathrm{t} 3935(3), \mathrm{t} 5708(3), \mathrm{t} 5983(3)\end{array}$ & ST22 (100) & ND & $\begin{array}{l}\text { Multiple } \\
\text { sites } \\
(2005-10)\end{array}$ \\
\hline$[7]$ & $209^{\mathrm{E}, \mathrm{F}}$ & $31 \%$ & $5 \%$ & $\begin{array}{l}\text { IV (40), III } \\
(34), \text { V (26), } \\
\text { VI (4) }\end{array}$ & $\begin{array}{c}\mathrm{t} 860(24), \mathrm{t} 945(9), \mathbf{t} 127(7), \mathrm{t} 688(7), \mathbf{t 3 0 4}(6), \mathrm{t} 044(6), \text { spa-NT } \\
(1), \mathbf{t 0 0 3}, \mathbf{t 0 0 2}, \mathbf{t 0 0 5}, \mathbf{t 0 0 8}, \mathbf{t} 018, \mathrm{t} 019, \mathbf{t 0 2 1}, \mathbf{t 0 3 2}, \mathbf{t} 037, \mathrm{t} 042, \\
\mathbf{t 0 4 5}, \mathrm{t} 148, \mathrm{t} 105, \mathrm{t} 1120, \mathrm{t} 11836, \mathrm{t} 14700, \mathrm{t} 1247, \mathrm{t} 12398, \mathrm{t} 16185, \\
\text { t1839, t16202, t148, t306, t314, t355, t359, t362, t363, t376, } \\
\text { t3841, t425, t535, t5414, t657, t6845, t690, t701, t713, t7583, } \\
\text { t790, t8154, t8168, t852, t084 }\end{array}$ & ST239 (27), ST2867 (1) & $\begin{array}{c}\text { CC8 (27\%), CC5 (18\%), CC1 (9\%), CC6 } \\
(9 \%), \text { CC22 (9\%), CC80 (7\%), CC30 (6\%), } \\
\text { CC97 (3\%), CC15 (3\%), CC152 (2\%), CC88 } \\
(2 \%), \text { CC361 (2\%), CC2250/2277 (1\%), } \\
\text { CC45 (1\%), CC121 (1\%), CC59 (1\%), } \\
\text { singleton ST2867 (1\%) } \\
\end{array}$ & $\begin{array}{l}\text { Farwaniya } \\
\text { h (2016) }\end{array}$ \\
\hline [73] & $1,327^{\mathbf{G}}$ & $45 \%$ & $24 \%$ & $\begin{array}{l}\text { IV (50), V } \\
(27), \text { III (9), } \\
\text { VI (8), I } \\
(0.1), \text { II (1) } \\
\end{array}$ & $\begin{array}{l}261 \text { identified; t688, t304, t860, t127, t044, t311, t002, t223, t267, } \\
\text { t019, t3841, t005, t084, t852, and t657 (collectively made } 51 \% \text { ) }\end{array}$ & ND & $\begin{array}{c}\text { CC361 (32), CC30 (15), CC22 (13), CC1 } \\
(11), \text { CC8 (6), CC152 (6), CC121 (6), CC913 } \\
(3), C C 45 \text { (2), CC88 (2), CC97 (2), CC1153 } \\
(1), \text { CC5 (1), CC6 (1), S-ST2867 (1) }\end{array}$ & $\begin{array}{l}\text { Multiple } \\
\text { sites } \\
(2016)\end{array}$ \\
\hline \multicolumn{9}{|c|}{ (n) } \\
\hline \multirow[t]{2}{*}{ [71] } & $\begin{array}{c}26 \\
(2003)^{\mathbf{H}}\end{array}$ & $27 \%$ & $4 \%$ & $\begin{array}{l}\text { II (19), III } \\
\text { (12), IIIa } \\
(12), \text { IV (54), } \\
\text { IVa (4) } \\
\end{array}$ & $\begin{array}{c}\text { t002 (23), t032 (19), t037 (16), t044 (12), t421 (8), t690 (8), t019 } \\
\text { (4), t359 (4), t064 (4), t878 (4) }\end{array}$ & $\begin{array}{c}\text { ST239 (23), ST5 (23), ST22 (19), ST80 (12), } \\
\text { ST88 (8), ST30 (4), ST97 (4), ST113 (4), } \\
\text { ST779 (4) }\end{array}$ & ND & \multirow{2}{*}{$\begin{array}{l}\text { Al-Ain } \\
(2003-8)\end{array}$} \\
\hline & $\begin{array}{c}26 \\
(2008)^{\mathbf{H}}\end{array}$ & $39 \%$ & $19 \%$ & $\begin{array}{l}\text { III (8), IV } \\
(69), \text { NT (23) }\end{array}$ & $\begin{array}{c}\text { t044 (27), t002 (16), t688 (12), t127 (12), t037 (8), t018 (4), t019 } \\
(4), \text { t304 (8), t005 (4), t1081 (4), t315 (4) }\end{array}$ & $\begin{array}{c}\text { ST80 (27), ST5 (27), ST1 (12), ST239 (8), } \\
\text { ST30 (8), ST6 (8), ST22 (4), ST45 (4), ST361 } \\
\text { (4) }\end{array}$ & ND & \\
\hline [88] & $57 \mathrm{CO}$ & $100 \%$ & ND & IV (100) & ND & ND & ND & $\begin{array}{c}\text { Sharjah } \\
(2011-12)\end{array}$ \\
\hline \multicolumn{9}{|c|}{ Qatar } \\
\hline [11] & 61 & $66 \%$ & ND & $\begin{array}{l}\text { IV }(90), \mathrm{V} \\
\quad(10)\end{array}$ & $\begin{array}{l}\text { t019 (28), t002 (21), t044 (13), t852 (13), spa-NT (8), t008 (3), } \\
\text { t121 (3), t690 (3), t267 (2), t657 (2), t7358 (2), t314 (2) }\end{array}$ & $\begin{array}{l}\text { ST30 (28), ST5 (21), ST80 (13), ST22 (13), } \\
\text { ST8 (12), ST15 (5), ST88 (3), ST-NT (3), }\end{array}$ & $\begin{array}{l}\text { Based on PFGE: Southwest pacific (28), } \\
\text { Pediatric (21), UK-EMRSA-15 (13), } \\
\text { European (13), USA300 (7), USA900 (5), } \\
\text { USA400 (5), USA1200 (2), Unknown (7) }\end{array}$ & $\begin{array}{c}\text { Doha } \\
(2009-10)\end{array}$ \\
\hline \multicolumn{9}{|c|}{ Bahrain } \\
\hline$[6]$ & 53 & ND & ND & $\begin{array}{l}\text { III (87), IV } \\
\quad(13)\end{array}$ & ND & ND & PFGE: A (83), C (6), D (6), B (2), E (2) & $\begin{array}{l}\text { Manama } \\
(2005)\end{array}$ \\
\hline \multicolumn{9}{|c|}{ Gaza } \\
\hline [45] & 121 & $42 \%$ & $40 \%$ & $\begin{array}{l}\text { I (3), III (7), } \\
\text { IV (2), IVa } \\
(49), \text { IVc } \\
(29), \text { V (7), } \\
\text { VI (3) } \\
\end{array}$ & $\begin{array}{c}\text { t044 (30), t223 (28), t7063 (9), t318 (8), t037 (8), t008 (6), t309 } \\
(2), \mathrm{t} 541(2), \mathrm{t} 5485(2), \mathrm{t} 2229(2), \mathrm{t} 001(2), \mathrm{t} 688(2), \mathrm{t} 14480(2), \\
\text { t7793 (2), t903 (1), t458 (1), t4229 (1) }\end{array}$ & $\begin{array}{l}\operatorname{ST22}(41), \operatorname{ST} 80(31), \operatorname{ST} 8(8), \operatorname{ST} 30(8) \\
\text { ST239 (7), ST228 (2), ST5 (1), ST6 (1), } \\
\text { ST121 (1), ST1153 (1) }\end{array}$ & $\begin{array}{c}\mathbf{C C 2 2}(41), \mathbf{C C 8 0}(31), \mathbf{C C 8}(8), \mathbf{C C 3 0}(8), \\
\operatorname{CC} 239(7), \operatorname{CC5}(3), \operatorname{CC6}(1), \operatorname{CC} 121(1 \%), \\
\operatorname{CC} 1153(1)\end{array}$ & $\begin{array}{c}\text { Gaza } \\
(2008-12)\end{array}$ \\
\hline \multicolumn{9}{|c|}{$\begin{array}{ll} & \text { Jordan } \\
\end{array}$} \\
\hline [47] & 41 & $37 \%$ & ND & ND & $\begin{array}{c}\mathbf{t 0 4 4}(38), \mathbf{t 0 3 7}(11), \mathrm{t} 318(5), \mathrm{t} 386(5), \mathrm{t} 1149(5), \mathrm{t} 701(3), \mathrm{t} 5849 \\
(3), \mathrm{t} 318(3), \mathrm{t} 6438(3), \mathrm{t091}(3), \mathrm{t} 6432(3), \mathbf{t 0 1 2}(3), \mathrm{t} 743(3), \\
\text { t6439(3), t6303 (3), t363 (3), t159(3), t5802 (3) }\end{array}$ & ND & ND & $\begin{array}{l}\text { Amman } \\
(2009-10)\end{array}$ \\
\hline [81] & $\begin{array}{l}31 \mathrm{Spa}- \\
\mathrm{CC} 044\end{array}$ & $48 \%$ & $58 \%$ & ND & t044 (83), t5849 (6), t131 (3), t5802 (3). t6438 (3) & ST80 (97), ST997(3) & CC80 $(100 \%)$ & $\begin{array}{l}\text { Amman } \\
(2000-11)\end{array}$ \\
\hline
\end{tabular}




\begin{tabular}{|c|c|c|c|c|c|c|c|c|}
\hline \multicolumn{9}{|c|}{ Lebanon } \\
\hline [48] & 93 & $62 \%$ & ND & $\begin{array}{c}\text { II (1), III } \\
\text { (10), IVb (1), } \\
\text { IVc (87), V } \\
\text { (1) }\end{array}$ & $\begin{array}{c}\mathbf{t 0 4 4}(53), \mathbf{t 0 3 7}(6), \text { t030 (4), } \mathbf{t 0 0 8}(4), \mathrm{t} 267(4), \mathbf{t 3 0 4}(3), \mathbf{t 0 3 2}(3), \\
\text { t131 (2), t311 (1), t318 (1) }\end{array}$ & $\begin{array}{l}\text { ST80 (55), ST239 (11), ST8 (4), ST97 (4), } \\
\text { ST6 (3), ST22 (3), ST5 (1), ST30 (1) }\end{array}$ & $\begin{array}{c}\text { CC80 (55), CC8 (15), CC97 (4), CC6 (3\%), } \\
\text { CC22 (3), CC5 (1), CC30 (1) }\end{array}$ & $\begin{array}{c}\text { Beirut } \\
(2006-7)\end{array}$ \\
\hline [81] & $\begin{array}{l}63 \text { spa- } \\
\text { CC044 }\end{array}$ & $92 \%$ & $0 \%$ & IV $(100)$ & t044 (85), t131 (8), t4222 (2), t6438 (2), t021 (2), t9135 (2) & ST80 (100) & CC80 (100\%) & $\begin{array}{l}\text { Beirut } \\
(2000-11)\end{array}$ \\
\hline \multicolumn{9}{|c|}{ Iraq } \\
\hline [74] & $182^{I}$ & $96 \%$ & ND & $\begin{array}{c}\text { IV (90), III } \\
\text { (3), I (1), NT } \\
\text { (4) }\end{array}$ & ND & ND & \begin{tabular}{|l} 
Based on PFGE: USA300 (80), USA100 (4), \\
USA1100 (3), USA400 (2), USA800 (2), \\
USA1000 (1), CSH5 (3), CSH1(1), CSH2 \\
(1), CSH3 (1), CSH4(1), CSH6 (1), CSH7(1), \\
CSH8(1), CSH9 (1), CSH10 (1), CSH11 (1) \\
\end{tabular} & $\begin{array}{l}\text { Multiple } \\
\text { sites } \\
(2007-9)\end{array}$ \\
\hline \multicolumn{9}{|c|}{ Egypt } \\
\hline [55] & 12 & ND & $25 \%$ & ND & ND & ND & ND & $\begin{array}{c}\text { Ismailia } \\
(2013)\end{array}$ \\
\hline [56] & $18 \mathrm{CO}$ & $33 \%$ & ND & $\begin{array}{l}\text { V (50), NT } \\
\text { (33), IV (11), } \\
\text { IVc (6) }\end{array}$ & ND & ND & ND & $\begin{array}{c}\text { Alexandr } \\
\text { a (NR) }\end{array}$ \\
\hline [54] & 18 & $83 \%$ & ND & $\begin{array}{l}\text { II (33), V } \\
\text { (17), IVd } \\
\text { (17), III (11), } \\
\text { IVa (6), IVc } \\
\text { (6), NT (11) }\end{array}$ & ND & ND & ND & $\begin{array}{l}\text { Zagazig } \\
\text { and } \\
\text { Assuit } \\
(2010-12)\end{array}$ \\
\hline \multicolumn{9}{|c|}{ Algeria } \\
\hline [58] & 92 & $21 \%$ & ND & $\begin{array}{l}\text { III (78), IVc } \\
\quad(22)\end{array}$ & t037 (43), t932 (35), t044 (21), t005 (1) & ST239 (78), ST80 (21), ST22 (1) & CC8 (78), CC80 (21), CC22 (1) & $\begin{array}{c}\text { Annaba } \\
(2010)\end{array}$ \\
\hline \multirow[b]{2}{*}{ [59] } & $15 \mathbf{H O}^{\mathbf{J}}$ & $100 \%$ & ND & IVc (100) & ND & ST80 (100) & ND & \multirow[b]{2}{*}{$\begin{array}{c}\text { Algiers } \\
(2010-11)\end{array}$} \\
\hline & $10 \mathbf{C O}^{\mathrm{J}}$ & $70 \%$ & ND & $\begin{array}{l}\text { II (10), IVc } \\
\quad(90)\end{array}$ & ND & ST80 (90), ST39 (10) & ND & \\
\hline \multirow{2}{*}{ [61] } & $84 \underset{\mathrm{L}}{\mathbf{C} \mathbf{C O}^{\mathrm{K}}}$ & $88 \%$ & $0 \%$ & $\begin{array}{l}\text { IV (97), V } \\
(6), \text { III (3) }\end{array}$ & t044 (85), t4143 (3) & ST80 (91), ST8 (6), ST241 (3) & ND & \multirow{2}{*}{$\begin{array}{c}\text { Algiers } \\
(2006-7)\end{array}$} \\
\hline & $\begin{array}{c}137 \\
\mathbf{H O}^{\mathbf{K}, \mathbf{L}} \\
\end{array}$ & $75 \%$ & $0 \%$ & $\begin{array}{c}\text { IV (88), III } \\
(12)\end{array}$ & t044 (96) & ST80 (89), ST5 (11) & ND & \\
\hline [75] & 84 & $86 \%$ & ND & $\begin{array}{l}\text { IV (88), III } \\
\text { (8), II (1), } \\
\text { ND (2) }\end{array}$ & ND & ST80 (86), ST239 (8), others (6) & ND & $\begin{array}{c}\text { Algiers } \\
(2006-11) \\
\end{array}$ \\
\hline [76] & 64 & $30 \%$ & $0 \%$ & $\begin{array}{l}\text { IV (50), V } \\
(44)\end{array}$ & ND & ND & ND & $\begin{array}{l}\text { Constanti } \\
\text { ne (2005- } \\
7 \text { 7) }\end{array}$ \\
\hline [60] & $73^{\mathrm{M}}$ & $15 \%$ & $0 \%$ & ND & ND & ST239 (82), ST80 (14), ST-NT (4) & ND & $\begin{array}{r}\text { Annaba } \\
(2011-12) \\
\end{array}$ \\
\hline
\end{tabular}




\begin{tabular}{|c|c|c|c|c|c|c|c|c|}
\hline [64] & 58 & $36 \%$ & ND & ND & ND & ND & ND & $\begin{array}{c}\text { Tunis } \\
(2005-7)\end{array}$ \\
\hline [65] & $\begin{array}{c}24 \\
\text { tetracycli } \\
\text { ne } \\
\text { resistant }\end{array}$ & $4 \%$ & $0 \%$ & ND & $\begin{array}{c}\mathrm{t} 052(5), \mathrm{t} 037(21), \mathrm{t} 14712(4), \mathrm{t} 129(4), \mathrm{t} 044(8), \mathrm{t} 311(4), \mathrm{t} 7144 \\
\text { (4), t899 (4) }\end{array}$ & $\begin{array}{l}\text { ST247 (50), ST239 (25), ST728 (8), ST241 } \\
\text { (4), ST641 (4), ST7144 (4), ST398 (4) }\end{array}$ & CC8 (80), CC80 (8), CC5 (8), CC398 (4) & $\begin{array}{c}\text { Tunis } \\
(2011-12)\end{array}$ \\
\hline \multirow[b]{2}{*}{ [77] } & $28 \mathbf{C O}^{\mathbf{N}}$ & $79 \%$ & ND & $\begin{array}{l}\text { IVc (79), NT } \\
(18), \text { I (4) }\end{array}$ & ND & $\begin{array}{c}\text { ST80 (74), ST5 (8), ST153 (8), ST2563 (4), } \\
\text { ST1 (4), ST45 (4) }\end{array}$ & CC80 (86), CC5 (7), CC1 (4), CC45 (4) & \multirow[b]{2}{*}{$\begin{array}{l}\text { Tunis } \\
(2004-8)\end{array}$} \\
\hline & $41 \mathbf{H O}^{\mathbf{N}}$ & $51 \%$ & ND & $\begin{array}{c}\text { I (15), III } \\
(17), \text { IVc } \\
(56), \text { NT }(12) \\
\end{array}$ & ND & $\begin{array}{l}\text { ST80 (49), ST239 (10), ST241 (7), ST247 (7) } \\
\text { ST1819 (7), ST5 (7), ST97 (5), ST1440 (2), } \\
\text { ST1 (2), ST22 (2) }\end{array}$ & $\begin{array}{c}\mathbf{C C 8 0}(51), \mathbf{C C 8}(32), \mathbf{C C 5}(7), \operatorname{CC} 15 \text { (5), } \\
\text { CC1 (2), CC22 (2) }\end{array}$ & \\
\hline
\end{tabular}

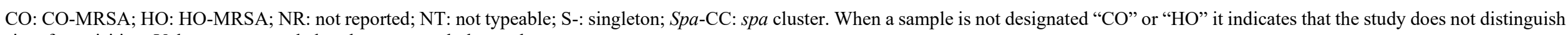
site of acquisition. Values were rounded to the nearest whole number.

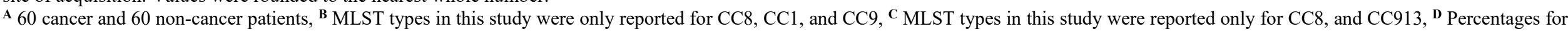

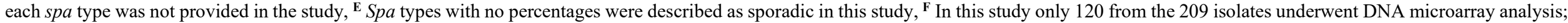

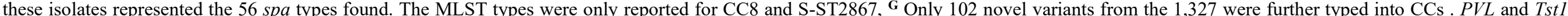

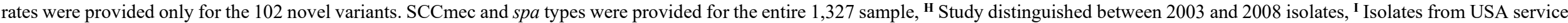

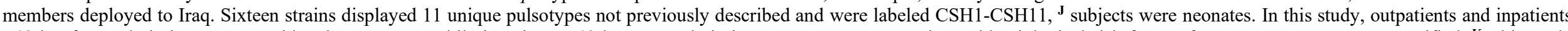

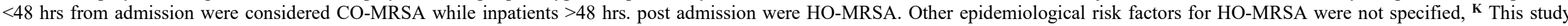

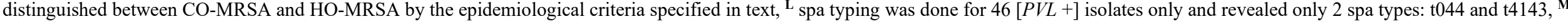
Diabetic foot infections, ${ }^{\mathrm{N}}$ Criteria used to distinguish between HO-MRSA and CO-MRSA is not specified. 
had SCCmec-III. Spa types t037, t030, and t008 were most commonly seen. ${ }^{12,16,20,23,26,27,31,34}$

The USA300 CA-MRSA was overall less commonly encountered than the Brazilian/Hungarian clone. It was isolated infrequently in KSA, ${ }^{67,68}$ Kuwait, ${ }^{69,70}$ Qatar, ${ }^{11}$ Gaza, ${ }^{45}$ and Lebanon. ${ }^{48}$ In Kuwait, it accounted for $8 \%$ of 26 SCCmec-IV MRSA isolates only, as opposed to the European CA-MRSA, which made up 46\%. Nonetheless, USA300 was very common in Iraq, where it accounted for $80 \%$ of infective isolates from deployed U.S. soldiers. ${ }^{74}$ The clinical specimen was $95 \%$ from wound infections. Most non-wound isolates were not USA300. Almost all USA300 isolates were $[P V L+]$. Despite being in separate geographical locations, similar pulsotypes were identified in the 3 combat support hospitals (CSH), which indicated the same origin of MRSA. ${ }^{74}$ It is possible that soldiers were colonized before deployment as USA300 is a prevailing clone in the U.S. ${ }^{8}$

Finally, a [PVL-] CC8-V-t008 clone was detected in few isolates from KSA, ${ }^{12}$ Egypt, ${ }^{12}$ and Kuwait. ${ }^{73}$ This clone was previously described in Saxony and Australia. ${ }^{8}$ Clonal complex 22: This complex contains multiple MRSA lineages. ${ }^{8}$ The best example is the ST22SCCmec-IV pandemic clone, also known as UKEMRSA-15 HA-MRSA. When present, it tends to become abundant. It is considered HA-MRSA but can also disseminate in the community. In previous reports, it accounted for $50 \%-80 \%$ of isolates from countries in Europe. It is typically [PVL-] and [Tst1-], but variants carrying either or both toxins exist. For example, $[P V L+]$ ST22-SCCmec-IV was previously described in a large hospital outbreak in Germany. ${ }^{8}$ This variant was also detected in other parts of Europe. A $[P V L+, T s t 1+]$ variant was previously identified in India. ${ }^{8}$ Investigators hypothesize that these variants could have a polyphyletic origin since they can exist in epidemiologically unrelated settings. A $[P V L-]$ SCCmec-V clone was also infrequently detected in Saxony and western Australia. CC22-SCCmec-I, II, or III are not described in the literature. $^{8}$

[PVL-] ST22-SCCmec-IV or its related spa types were commonly reported in colonizing strains from the MENA region, ${ }^{12,16,20,23,27,31,34}$ particularly common in Gaza, Jordan, Egypt, and Algeria. ${ }^{12,23,27,31,34}$ This clone was also prevalent in infective strains from the GCC and Gaza. It was reported in $10 \%-28 \%$ of isolates from $\mathrm{KSA}^{67,68} 13 \%$ in Qatar, ${ }^{11}$ and $9 \%$ in Kuwait. ${ }^{7,69,73}$ In UAE, it accounted for $19 \%$ of isolates in 1 center in 2003 but then dropped to $4 \%$ in 2008 , showing a decreasing presence with time while other clones dominated. ${ }^{71}$ The ST22-SCCmec-IV clone was exceptionally common in Gaza, where it made up $41 \%$ of isolates in 1 study. On the other hand, this clone was infrequently encountered in the other Levant and North African countries. ${ }^{47,48,58-}$ $61,65,74,75,77$
All the CC22 isolates from the MENA carried either SCCmec-IV or V. The most common spa types were t223 and t032. The majority were [PVL-] as expected. Nonetheless, the $[P V L+]$ variant was detected in KSA, Kuwait, and Qatar, where, in some centers, this variant exceeded the [PVL-] ST22 strains. ${ }^{11,68,69}$ Furthermore, many isolates from KSA and Kuwait were [Tst1+], also known as the "Middle Eastern" variant. In Kuwait, 62\% of a subset of urease (-) EMRSA-15 isolates belonged to the Middle Eastern variant. ${ }^{78}$ This variant mostly carried SCCmec-IVa and was $[P V L-]$, except for some isolates from Kuwait, $[P V L+]$ indicating the potential to acquire both toxins. Notably, the bulk (91\%) of infective isolates in Gaza were $\left[T_{s t 1+] .}{ }^{45}\right.$ Nonetheless, these were collectively referred to as the "Gaza" strain. ${ }^{45}$ The Gaza strain also exists in Jordan where it was dominant in colonizing strains in 1 study. ${ }^{27}$ On the other hand, it was not detected in studies from Lebanon. ${ }^{48,79}$ The Gaza strain was associated with the SCCmec-IVa-t223 genotype. Like the Middle Eastern variant, the Gaza strain was mostly [PVL-].

While the PFGE analysis of the Gaza strain in Gaza showed $76 \%$ pattern similarity with UK-EMRSA-15, some distinguishing features exist. The UK-EMRSA-15 is associated with SCCmec-IVh-t032 and t022 instead of SCCmec-IVa-t223. ${ }^{23,27}$ Furthermore, Unlike UKEMRSA-15, the Gaza strain is susceptible to ciprofloxacin and resistant to tetracycline. ${ }^{23,27}$ Some of the ST22-SCCmec-IV strains in GCC and North African Arab regions also carried SCCmec-IVa-t223 distinguishing them from UK-EMRSA-15 clone. ${ }^{12,23,27,34,45,78}$ One study examined 47 CC22SCCmec-IV isolates from patients from KSA, Abu Dhabi, Kuwait, and Germany. ${ }^{80}$ Six distinct albeit related strains were detected. Only 9 isolates showed complete resemblance to the UK-EMRSA-15. ${ }^{80}$ The study concluded that none of the isolates from Riyadh were true UK-EMRSA-15. True UK-EMRSA-15, however, was identified in Abu Dhabi and Kuwait. ${ }^{80}$

Lastly, [PVL-] CC22-SCCmec-V was detected in few strains from KSA and Gaza. ${ }^{12,23,45}$ This clone carried $\mathrm{t} 223$ and 77063 spa types and was [Tst1+] in Gaza.

Clonal complex 30: Important clones include UKEMRSA-16 HA-MRSA (ST36/39-SCCmec-II) and Southwest Pacific CA-MRSA ([PVL+] ST30-SCCmecIV). ${ }^{8}$ The former was an important clone in Europe but is becoming rare. The latter is widespread globally. A $[P V L-$, Tst $1+] \mathrm{ST} 30-\mathrm{SCCmec-IV}$ is sporadically isolated from Ireland and Australia. ${ }^{8}$

Overall, CC30 was only occasionally encountered in most of the countries in the region. It was detected in few colonizing isolates from Gaza, ${ }^{23}$ Jordan, ${ }^{27}$ and Egypt. ${ }^{12}$ Most colonizing isolates were $[P V L-]$, and in Jordan, several of them were $[T s t 1+]$ It was also rarely seen in infective isolates from $\mathrm{KSA}^{68}{ }^{6} \mathrm{Kuwait}^{69}$ and UAE, ${ }^{71}$ 
Gaza, ${ }^{45}$ Lebanon, ${ }^{48}$ and Jordan. ${ }^{47}$ None of the studies from Algeria and Tunisia isolated CC $30{ }^{65,77}$ However, this was the most common clone in infective isolates from 1 center in Qatar. ${ }^{11}$ Also, it made up 31\% of a sample of 26 SCCmec-IV isolates in Kuwait. ${ }^{70}$ Almost all isolates from the region carried SCCmec-IV, resembling Southwest pacific CA-MRSA. ${ }^{27}$ Associated spa types were t019 and $\mathrm{t} 018$ in the GCC, $, 8,69,71$ and $\mathrm{t} 318$ in the Levant. ${ }^{45,47,48}$ Unlike colonizing strains, all infective isolates from this region were $[\mathrm{PVL}+]$ except for 1 in Jordan.

The UK-EMRSA-16 HA-MRSA was also rarely reported in the region; only 8 isolates from Kuwait. ${ }^{69}$ Finally, when isolates carrying novel genotypes in Kuwait were tested, $15 \%$ carried CC $30-$ SCCmec-VI. $^{73}$ Clonal complex 80: This complex houses the European CA-MRSA clone (ST80-SCCmec-IV), which gains its name due to its significant presence throughout Europe. However, this clone is encountered worldwide; most isolates belonging to CC80 carry $[P V L+]$ ST80SCCmec-IV and IV variants. $[P V L-]$ strains are infrequently detected.

ST80-SCCmec-IV or the associated spa types were detected in colonizing strains from all recovered studies. $^{12,16,20,23,27,31,34}$ Among infective strains, it was detected in $20-30 \%$ of isolates from $\mathrm{KSA},{ }^{67,68} 7 \%$ in Kuwait, ${ }^{7,69}$ and $13 \%$ in Qatar. ${ }^{11}$ In UAE, one center experienced a rise in rates from $12 \%$ in 2003 to $27 \%$ in $2008 .^{71}$ When examining a sample of SCCmec-IV isolates in Kuwait, $46 \%$ were ST 80 -SCCmec-IV. ${ }^{70}$ This clone was even more prevalent in infective isolates from the Levant and North Africa. It made up 31\% of isolates in Gaza, ${ }^{45}$ and 55\% in Lebanon. ${ }^{48}$ In Jordan, $[P V L+]$ t044 and $[P V L-]$ t044 were detected in $27 \%$ and $11 \%$ of isolates, respectively. ${ }^{47}$ This clone was completely absent in an Iraqi study examining clinical isolates from U.S. soldiers. ${ }^{74}$ In Algeria, this was the most frequently encountered clone in Algiers, $77 \%-100 \%$ of isolates..$^{59,61,75}$ This was true even for HO-MRSA isolates, indicating the infiltration of this CA-MRSA clone into the hospital setting. However, its presence was less frequently encountered in studies from a different city, Annaba ${ }^{58,60}$ In Tunisia, it accounted for $51 \%$ of HOMRSA and $86 \%$ of CO-MRSA. ${ }^{77}$

There is evidence that isolates belonging to European CA-MRSA in the region could have different evolutionary lineages. For example, PFGE typing revealed 21 different clonal groups among spa-clonalcluster-044 isolates from Lebanon and Jordan. ${ }^{81}$ Moreover, the $P V L$ gene was detected more frequently in the Lebanese subset, while the Tst1 gene was detected only in the Jordanian subset. ${ }^{81}$

The $[P V L-]$ variant was infrequently encountered in KSA, ${ }^{67}$ Kuwait, ${ }^{69,82}$ Jordan, ${ }^{47,81}$ Lebanon $^{81}$, Algeria, ${ }^{59,61}$ and Tunisia. ${ }^{65,77} \mathrm{~A}\left[T_{s t 1+]}\right.$ variant was detected in Kuwait, ${ }^{82}$ Gaza, ${ }^{45}$ and Jordan. ${ }^{81}$ On the other hand, all strains in $\mathrm{UAE},{ }^{71}$ Qatar, ${ }^{11}$ Gaza ${ }^{45}$ were $[P V L+]$. The most encountered spa type was t044. ${ }^{45,48,58,61,65,81}$

Other Clonal Complexes: CC15 was detected in a small number of colonizing isolates from KSA, ${ }^{12}$ Jordan, ${ }^{27}$ and Egypt. $^{31}$ These were carrying t083, t084, and t085. It was also seen in some infective strains from KSA, ${ }^{68}$ Kuwait, $^{7}$ and Tunisia. ${ }^{77}$ This CC is rarely described. ${ }^{8}$ It was detected in a collection of strains from Italy in 1980, 4 SCCmec-I isolates. ${ }^{8}$ More recently, it was reported in Europe, Iran, Gabon, and the Gulf of Guinea. ${ }^{12}$ In Europe, it accounted for $17 \%$ of MRSA and methicillinsusceptible (MSSA) isolates combined and carried ST1, ST15, ST188, ST772, and ST1835. In KSA, isolates of CC15 carried both SCCmec-IVa and V. ${ }^{12}$

In Jordan, a novel genotype, [PVL-,Tst 1+]-IVe-t9519, made up the bulk of colonizing isolates from Amman. ${ }^{26}$

There are still other CCs and clones identified in the region but seem to be of lower prevalence compared to the 5 main CCs we chose to discuss.

Antimicrobial Resistance Patterns of MRSA in the MENA Region: An in-depth description of the antimicrobial susceptibility of MRSA in the MENA region is not the primary objective of our review. Therefore, we do not discuss this topic in detail. Table 6 lists cumulative susceptibilities for 462 colonizing and 11, 373 infective MRSA isolates per country. Supplementary Table 5 provides the susceptibilities from individual studies. The susceptibility ranges are wide for some antimicrobials, indicating a significant difference in resistance patterns between different centers within a country.

Antimicrobial Resistant patterns of Colonizing MRSA: Studies from GCC and North African Arab countries showed high resistance to TMP-SMX..$^{12,14,19,20,34,83}$ On the other hand, all isolates from the Levant were susceptible to this agent. ${ }^{23,29}$ For ciprofloxacin, isolates from North Africa and Levant were largely sensitive as opposed to the GCC. . $^{12,14,19,20,23,27,29,34}$ Nonetheless, studies from Gaza and Jordan showed that high resistance to ciprofloxacin could be encountered in isolates retrieved from healthcare workers. ${ }^{25,27}$ Furthermore, while some centers in KSA showed high resistance, others showed $100 \%$ susceptibility to ciprofloxacin. ${ }^{12,14}$ Almost all countries showed high resistance to tetracycline, clindamycin, and erythromycin. . $^{12,14,19-23,25,27,29,34}$ Isolates also remained highly susceptible to rifampin in most centers. Surprisingly, significant vancomycin resistance was detected in KSA, Oman, Egypt, and Gaza. ${ }^{12,14,21,22,25}$ The majority of these studies used Kirby Bauer's disk diffusion method using Mueller-Hinton agar ${ }^{12,14,22,25}$ except for 1 study, which used E-test for minimum inhibitory concentration (MIC). ${ }^{21}$ The remaining studies showed $100 \%$ susceptibility to vancomycin. ${ }^{19,20,23,27,29,34}$ Isolates were almost universally susceptible to linezolid except in 1 study from KSA. ${ }^{14}$ 
Table 6. Percent susceptibility of total MRSA isolates. Susceptibility presented as total \# isolates susceptible/total isolates tested (\% susceptible).

\begin{tabular}{|c|c|c|c|c|c|c|c|c|c|c|c|c|c|c|c|c|}
\hline Country & Ref (date) & \# MRSA & Rif & FA & $\mathbf{C l}$ & TS & Cip & Tet & Dox & EM & Vanc & Lin & Teic & Dap & Mup & Gen \\
\hline \multicolumn{17}{|c|}{ Colonizing MRSA strains- Susceptibility presented as total \# of susceptible isolates /total \# of tested isolates tested (\%) } \\
\hline \multirow{2}{*}{$\mathrm{Oman}^{\mathrm{A}}$} & {$[21,22]$} & \multirow{2}{*}{83} & \multirow{2}{*}{$81 / 83(98)$} & \multirow{2}{*}{ - } & \multirow{2}{*}{$58 / 83(70)$} & \multirow{2}{*}{-} & \multirow{2}{*}{ - } & \multirow{2}{*}{ - } & \multirow{2}{*}{ 75/83 (90) } & \multirow{2}{*}{$42 / 83(51)$} & \multirow{2}{*}{$73 / 83(88)^{\mathbf{B}}$} & \multirow{2}{*}{$83 / 83(100)$} & \multirow{2}{*}{ 69/83 (83) } & \multirow{2}{*}{ - } & \multirow{2}{*}{-} & \multirow{2}{*}{ - } \\
\hline & (NR) & & & & & & & & & & & & & & & \\
\hline \multirow{2}{*}{$\mathrm{KSA}^{\mathrm{A}}$} & {$[12,14,19]$} & \multirow{2}{*}{110} & \multirow{2}{*}{$53 / 74(72)$} & \multirow[b]{2}{*}{ - } & \multirow{2}{*}{$40 / 110(36)$} & \multirow{2}{*}{$60 / 110(55)$} & \multirow{2}{*}{$62 / 110(56)$} & \multirow{2}{*}{$39 / 110(35)$} & \multirow{2}{*}{ - } & 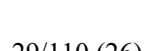 & $108 / 110$ & (O/O4 (e1) & 2610 (51) & & & 6217405 \\
\hline & $(2011-15)$ & & & & & & & & & $29 / 110(26)$ & $(98)^{\mathrm{B}}$ & $68 / 84(81)$ & $26 / 48(54)$ & - & - & $63 / 74(85)$ \\
\hline L ehanon ${ }^{A}$ & [29] & 8 & 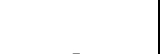 & & & Q/e (100) & e/e (100) & 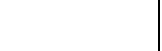 & & & e/e $(100)$ & & & & 0 & \\
\hline Lebanon & $(2006-7)$ & 8 & - & - & - & $8 / 8(100)$ & $8 / 8(100)$ & - & - & - & $8 / 8(100)$ & - & - & - & $8 / 8(100)$ & $8 / 8(100)$ \\
\hline$C=\sigma_{2}^{\mathrm{C}}$ & [23] & 145 & $15 / 51$ (e) & 80/04 (05) & $104 / 145$ & $04 / 04(100)$ & $138 / 145$ & 4451 (8) & & $01 / 145(63)$ & $137 / 145$ & & & & & $138 / 145$ \\
\hline Gaza & -2009 & 145 & $45 / 51(88)$ & $89 / 94$ (95) & $(72)$ & $94 / 94(100)$ & (95) & $44 / 31(80)$ & - & $91 / 145(03)$ & $(94)^{\mathbf{B}}$ & - & - & - & - & (95) \\
\hline $\mathrm{F}$ & [27] & 56 & $56156(100)$ & $50156(02)$ & $52156(05)$ & 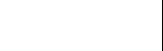 & $54156(06)$ & 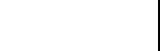 & & $24156(42)$ & $56156(100)$ & $56156(100)$ & $56156(100)$ & $56156(100)$ & $56156(100)$ & $56156(100)$ \\
\hline Jordan & $(2011-12)$ & 56 & $56 / 56(100)$ & $52 / 56(93)$ & $53 / 56(95)$ & - & $54 / 56(96)$ & - & - & $24 / 56(43)$ & $56 / 56(100)$ & $56 / 56(100)$ & $56 / 56(100)$ & $56 / 56(100)$ & $56 / 56(100)$ & $56 / 56(100)$ \\
\hline$F_{0}$ & {$[12]$} & 23 & $20 / 22$ (01) & & $92 / 23(67)$ & $17 / 22$ (50) & $22 / 22(1000)$ & 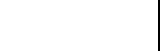 & & $15 / 22(15)$ & $20 / 22(01) \mathbf{B}$ & & & & & $202 / 23(67)$ \\
\hline Egypr & -2011 & 33 & $30 / 33(91)$ & - & $22 / 33(0 /)$ & $1 / / 33(32)$ & $33 / 33(100)$ & - & - & $15 / 33(45)$ & $30 / 33(91)^{-5}$ & - & - & - & - & $22 / 33(0 /)$ \\
\hline$A 1$ rorioA & {$[34]$} & 0 & $0 / 0(100)$ & & $0 / 0(100)$ & $7 / 0(78)$ & $0 / 0(100)$ & $6 / 0(67)$ & & $8 / 0$ (89) & $0 / 0(100)$ & $0 / 0(100)$ & $0 / 0(100)$ & & & $6 / 0(67)$ \\
\hline Argenta & $(2010-12)$ & 9 & $9 / 9(100)$ & - & $9 / 9(100)$ & $1 / 9(/ 8)$ & $9 / 9(100)$ & $6 / 9(6 /)$ & - & $8 / 9(89)$ & $9 / 9(100)$ & $9 / 9(100)$ & 9/9 (100) & - & - & $6 / 9(6 /)$ \\
\hline W. & [20] & 10 & 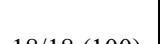 & (1) (20) & (1) (0) & $1940(70)$ & E 10 (1) & $5 / 10(0)$ & & (10 (1) & 1040 & 1040 & 1040 & & 7 & -50 \\
\hline Kuwatt $^{\mathrm{A}}$ & $(2005-7)$ & 18 & 18/18 (100) & 6/18(33) & $6 / 18(33)$ & $13 / 18(72)$ & $5 / 18(28)$ & $5 / 18(28)$ & - & $6 / 18(33)$ & $18 / 18(100)$ & $18 / 18(100)$ & $18 / 18(100)$ & - & $17 / 18(95)$ & $5 / 18(28)$ \\
\hline & & & & Infec & ive MRSA St & ains- Suscepti & oility presented & as total \# of su & sceptible isol & tes /total \# of & ested isolates t & sted (\%) & & & & \\
\hline$V \in \mathbb{D}$ & {$[66,72,85]$} & 723 & $170 / 221$ & $148 / 733$ & $136 / 221$ & $175 / 632$ & $62 / 101(60)$ & $58 / 101(57)$ & & $103 / 221$ & $733 / 733$ & $575 / 613$ & & & $565 / 733$ & $275 / 613$ \\
\hline $\mathrm{KSA}^{\circ}$ & $(2004-11)$ & 133 & $(77)$ & (20) & $(62)$ & (28) & 63/101 (62) & $58 / 101(57)$ & - & (47) & $(100)^{\mathbf{E}}$ & (94) & 89/101 (88) & - & (77) & (45) \\
\hline 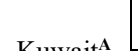 & {$[7,73,84]$} & 845 & $8389 / 8458$ & $4911 / 8458$ & $4911 / 8458$ & $5480 / 8458$ & $4859 / 8458$ & $5218 / 8458$ & & $4911 / 8458$ & $8389 / 8458$ & $8458 / 8458$ & $8250 / 8458$ & & $8054 / 8458$ & $4316 / 7131$ \\
\hline Nuwall & (2011-16) & $0,4 J 0$ & (99) & (58) & (58) & (65) & (57) & (62) & - & (58) & $(99)^{\mathbf{E}}$ & (100) & (98) & - & (95) & (61) \\
\hline Omon A & [43] & 732 & 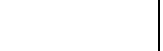 & & $571 / 733$ & $689 / 733$ & $506 / 733$ & 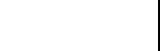 & & $520 / 733$ & $733 / 733$ & $733 / 733$ & & & & $623 / 733$ \\
\hline (IIIan & (2016-17) & (10) & 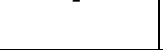 & - & (78) & (94) & (69) & - & - & (71) & $(100)$ & $(100)$ & - & - & - & $(85)$ \\
\hline BahrainA & {$[6]$} & 53 & $53 / 53(100)$ & $4 / 53(8)$ & $7 / 53(13)$ & $6 / 53(11)$ & $4 / 53(8)$ & $6 / 53(11)$ & - & $5 / 53(9)$ & $53 / 53(100)$ & $53 / 53(100)$ & $53 / 53(100)$ & $\ldots$ & $47 / 53(89)$ & $14 / 53(26)$ \\
\hline & -2005 & & & & & & & & & & & & & & & \\
\hline$I^{\prime}$ & [79] & 30 & $20 / 30(100)$ & & $34 / 30$ (07) & $38 / 30$ (07) & $34 / 30$ (87) & $27 / 30(60)$ & & $21 / 00$ & $20 / 100)$ & & $20 / 100)$ & & & \\
\hline Levalionti & -2011 & 39 & (I00) & - & $34 / 39(8 /)$ & $38 / 39(9 /)$ & $34 / 39(8 /)$ & $2 / 139(09)$ & - & $31 / 39(80)$ & $39 / 39(100)$ & - & $39 / 39(100)$ & - & - & - \\
\hline
\end{tabular}




\begin{tabular}{|c|c|c|c|c|c|c|c|c|c|c|c|c|c|c|c|c|}
\hline \multirow{2}{*}{$\mathrm{Gaza}^{\mathrm{F}}$} & {$[45]$} & \multirow{2}{*}{121} & \multirow{2}{*}{$\begin{array}{c}111 / 121 \\
(92)\end{array}$} & \multirow{2}{*}{-} & \multirow{2}{*}{$93 / 121(77)$} & \multirow{2}{*}{$97 / 121(80)$} & \multirow{2}{*}{ - } & \multirow{2}{*}{$82 / 121(68)$} & \multirow{2}{*}{ - } & \multirow{2}{*}{$86 / 121(71)$} & \multirow{2}{*}{$\begin{array}{c}121 / 121 \\
(100)\end{array}$} & \multirow{2}{*}{$\begin{array}{c}121 / 121 \\
(100)\end{array}$} & \multirow{2}{*}{$\begin{array}{c}121 / 121 \\
(100)\end{array}$} & \multirow{2}{*}{$\begin{array}{c}121 / 121 \\
(100)\end{array}$} & \multirow{2}{*}{$\begin{array}{c}121 / 121 \\
(100)\end{array}$} & \multirow{2}{*}{$97 / 121(80)$} \\
\hline & $(2008-12)$ & & & & & & & & & & & & & & & \\
\hline \multirow{2}{*}{ Jordan $^{\mathbf{A}}$} & {$[46]$} & \multirow{2}{*}{113} & \multirow[b]{2}{*}{$97 / 113(86)$} & \multirow[b]{2}{*}{-} & \multirow{2}{*}{$62 / 113(55)$} & \multirow{2}{*}{$66 / 113(58)$} & \multirow[b]{2}{*}{ - } & \multirow{2}{*}{$66 / 113(58)$} & \multirow{2}{*}{ - } & \multirow{2}{*}{$26 / 113(23)$} & \multirow{2}{*}{$\begin{array}{c}113 / 113 \\
(100)\end{array}$} & \multirow{2}{*}{$\begin{array}{c}110 / 113 \\
(97)\end{array}$} & \multirow{2}{*}{$\begin{array}{c}110 / 113 \\
(97)\end{array}$} & \multirow[b]{2}{*}{ - } & \multirow[b]{2}{*}{ - } & \multirow{2}{*}{$53 / 113(47)$} \\
\hline & $(2008-12)$ & & & & & & & & & & & & & & & \\
\hline \multirow{2}{*}{$\operatorname{Iraq}^{\mathbf{G}}$} & {$[51,52,74]$} & \multirow{2}{*}{500} & \multirow{2}{*}{$\begin{array}{c}462 / 500 \\
(92)\end{array}$} & & $379 / 500$ & $454 / 500$ & $158 / 318$ & $401 / 485(83)$ & & $66 / 500(13)$ & $485 / 485$ & $174 / 182$ & & & & $423 / 485$ \\
\hline & (2005-9) & & & - & (76) & (91) & $(50)$ & $401 / 485(83)$ & - & $66 / 500(13)$ & $(100)$ & (96) & - & - & - & (87) \\
\hline Foynta & {$[57]$} & 364 & $292 / 364$ & & $171 / 364$ & $302 / 364$ & $122 / 364$ & $58 / 364(16)$ & & $140 / 364$ & $364 / 364$ & & & & & $84 / 364(23)$ \\
\hline Lgypi & $(2005-13)$ & 304 & (80) & - & $(47)$ & (83) & (34) & $20 / 204(10)$ & - & (39) & (100) & - & - & - & - & $04 / 504(25)$ \\
\hline$A$ loriaA & {$[59,62]$} & 100 & $25 / 25,(100)$ & & $167 / 190$ & $24 / 25(100)$ & $20 / 25(80)$ & $605(24)$ & & & $187 / 190$ & & & & & $141 / 190$ \\
\hline Algeria & $(2007-11)$ & 190 & $25 / 25(100)$ & - & (88) & $24 / 25(100)$ & $20 / 25(80)$ & $6 / 25(24)$ & - & $91 / 165(55)$ & $(98)^{\mathbf{H}}$ & $25 / 25(100)$ & $25 / 25(100)$ & - & - & (74) \\
\hline Tuninina & {$[77]$} & 60 & 80 & & 84 & 01 & 50 & 17 & & 10 & 100 & & 100 & & & 74 \\
\hline I unisia & (2004-8) & 69 & 80 & - & 84 & 91 & 59 & 11 & - & 48 & 100 & - & 100 & - & - & 14 \\
\hline
\end{tabular}

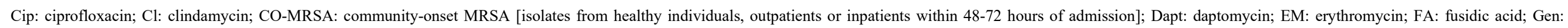

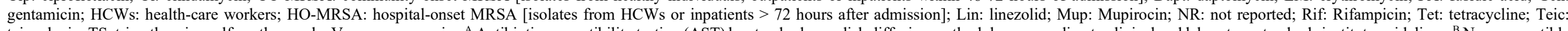

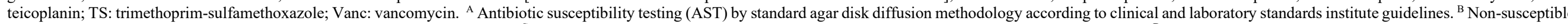

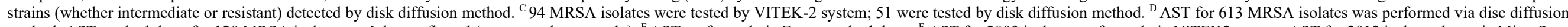

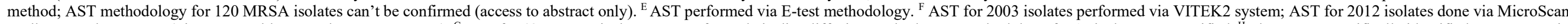

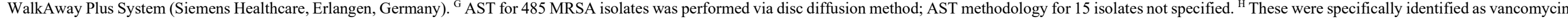
resistant and not vancomycin intermediate. 
Antimicrobial Resistant patterns of Infective MRSA: Infective MRSA in the MENA region were highly resistant against most antimicrobials. Susceptibility to TMP-SMX varied widely depending on the center. It reached acceptable to high levels in UAE and Oman in some studies. ${ }^{41,43,71}$ Strains from Lebanon and Iraq were highly sensitive to TMP-SMX as opposed to Gaza and Jordan. ${ }^{45,46,79}$ Nonetheless, the Gaza strain commonly encountered in Gaza was susceptible to TMP-SMX. ${ }^{23,27}$

Reported sensitivities to vancomycin, linezolid, and teicoplanin remained high among infective isolates in the region. ${ }^{6,25,41,43,45,46,57,59,66,71,77,79,84,85}$ Exceptionally, 5\% resistance to vancomycin was detected in 1 study from Egypt using the broth microdilution method. ${ }^{54}$

ST22-IV clones from GCC were highly susceptible to rifampin, vancomycin, linezolid, and teicoplanin. ${ }^{71,78}$ The UK-EMRSA- 15 and its $[P V L+]$ and $[P V L-]$ variants all had high susceptibility to tetracycline but low to ciprofloxacin. ${ }^{78}$ In contrast, [Tst1+] Middle Eastern variant was $100 \%$ sensitive to ciprofloxacin but only $70 \%$ to tetracycline. ${ }^{71,78}$ The "Gaza strain" was susceptible to most tested antimicrobials, including ciprofloxacin and TMP-SMX. ${ }^{23,27}$ It was often resistant to tetracycline. When compared to ST22-IV isolates from GCC, similar susceptibility rates were seen with few exceptions. The Middle Eastern variant was similarly sensitive to ciprofloxacin but, unlike the Gaza strain, was resistant to TMP-SMX. ${ }^{78}$ On the other hand, the $\left[P V L^{+}\right]$and $[P V L-]$ variants in $\mathrm{GCC}$ were similarly sensitive to TMP-SMX but differed due to high resistance to ciprofloxacin and high sensitivity to tetracycline. ${ }^{71,78}$

ST239 isolates from UAE were sensitive to vancomycin, linezolid, teicoplanin but resistant to other tested agents. ${ }^{71}$ In Egypt, they were highly resistant to tested antibiotics such as TMP-SMX, tetracycline, and gentamicin. ${ }^{58}$

ST80-IV CA-MRSA strains in GCC had high susceptibility rates to rifampin, TMP-SMX, vancomycin, linezolid, teicoplanin, and gentamicin..$^{25,71,82}$ Furthermore, the majority of Spa-clonal cluster-044 isolates from the Levant were susceptible to rifampin and gentamicin, ${ }^{81}$ which was consistent with GCC reports. ${ }^{71,82}$ The Lebanese isolates were often resistant to tetracycline, while the Jordanian isolates showed significantly higher resistance to erythromycin. ${ }^{81}$ In Egypt, this strain was often susceptible to TMP-SMX but resistant to tetracycline. ${ }^{58}$

Discussion. This review highlights a significant threat imposed by MRSA on both the community and healthcare settings within the Arab world. The rates of MRSA varied depending on the country and center. A significant spread of MRSA in some Arab communities was evident by the high nasal MRSA carriage rate among healthy outpatients, hospital visitors, or university students seen in certain cities. ${ }^{12,21-23}$ As expected, HCWs had higher nasal MRSA rates. $15,19,21,22,25,30$

In the clinical setting, methicillin resistance was highly prevalent among clinical isolates of $S$. aureus in the region. ${ }^{4,11,42,45-53,56-59,61,71,85}$ Thus, while some countries like UAE, Lebanon, Qatar, and Tunisia showed lower cumulative rates compared to others, there is still evidence of a high burden in some centers within these countries.

Regional variability in MRSA rates has been detected in other areas of the world, such as Europe. ${ }^{3,11,86}$ In the Arab world, this variance in rates could be due to differences in antibiotic prescribing practices and infection control efforts across the region and within each country. Additionally, Arab countries of the Levant and North Africa have suffered decades of economic and political instability, which negatively impacted medical resources and healthcare infrastructure instead of the prospering Arab countries in the GCC region. This could explain the lower rates seen in UAE and Qatar. Nonetheless, such a theory is challenged by the high MRSA rates seen in KSA. Perhaps these factors are offset by other determinants in KSA.

Certain genotypes were particularly prevalent in the Arab world, many of which belonged to 5 CCs known to spread worldwide. These were previously discussed in table 1. This is not surprising given that the Arab World is a center for multinational ex-pats seeking jobs abroad. Furthermore, KSA is the home of al-Ka'bah alMusharrafah, the destination of Muslim pilgrimage, where masses of Muslims from across the globe crowd up every year to perform religious rituals. These crowded conditions are likely to facilitate the spread of various MRSA strains.

Three clones were particularly important: European CA-MRSA, UK-EMRSA-15 HA-MRSA, and Brazilian/Hungarian HA-MRSA. Genotypes linked to these clones were distributed across the region. Notably, HA-MRSA clones might be decreasing in prevalence in some centers while CA-MRSA takes over. With time, a shift in clonal distribution was noted in Kuwait and UAE, where CA-MRSA genotypes became more prevalent. ${ }^{69,71,73,84}$ In Kuwait, this was clearly seen in 3 studies examining large samples from several hospitals across the country from 1992 to $2016 .{ }^{69,73,84}$ This is particularly true of the European CA-MRSA, which was detected in almost all cited studies and often accounted for a significant proportion of the study sample. Hence, this clone or its related strains seem to be infiltrating into the Arab world. Nonetheless, the presence of HA-MRSA clones remained strong in many studies, including both the Brazilian/Hungarian clone and the UK-EMRSA-15 clone.

USA300 CA-MRSA is another important clone that made up the bulk of isolates in Iraq's CSHs. However, the subjects here were U.S. military personnel and might 
have been colonized prior to being deployed. Hence this might not be reflective of the Iraqi population.

It is important to acknowledge distinguishing features when comparing local strains with these epidemic/pandemic clones. Strains from the Arab world have distinguishing antimicrobial resistance, SCCmec subtypes, and virulence factors. ${ }^{82}$ This was highlighted briefly during our discussion of $\mathrm{CC} 22$ in section Clonal complex 22. Another distinction was previously made between ST80-SCCmec-IV strains from the Arab world and the European CA-MRSA clone. ${ }^{81,82}$ The European CA-MRSA is known to be $[P V L+]$ and resistant to tetracycline, streptomycin, kanamycin, and fusidic acid (TSKF profile).$^{81}$ Overall, only $16 \%$ of the ST 80 isolates recovered from Lebanon and Jordan had this profile. ${ }^{81} \mathrm{~A}$ more commonly detected pattern in both countries included resistance to streptomycin, kanamycin, and fusidic acid (SKF pattern). ${ }^{81}$ Phenotypical variation between the Kuwaiti ST80 isolates and the European CA-MRSA clone was pointed out, particularly regarding some differences in antimicrobial resistance patterns and enterotoxin gene profiles. ${ }^{82}$ PFGE analysis of ST80 isolates from a subset of these hospitals in Kuwait showed that a large proportion was related but not identical, further pointing out the heterogeneity among local strains. ${ }^{82}$ This was mirrored in another study from Kuwait, which identified 102 novel variants of previously known genotypes reported locally and worldwide. ${ }^{73}$ Furthermore, it was shown that isolates derived from different regions in the Arab world could

\section{References:}

1. Deurenberg, R.H., et al., The molecular evolution of methicillin-resistant Staphylococcus aureus. Clin Microbiol Infect, 2007. 13(3): p. 222-35. https://doi.org/10.1111/j.1469-0691.2006.01573.x

2. Grundmann, H., et al., Emergence and resurgence of meticillin-resistant Staphylococcus aureus as a public-health threat. Lancet, 2006. 368(9538): p. 874-85.

https://doi.org/10.1016/S0140-6736(06)68853-3

3. Borg, M.A., et al., Prevalence of methicillin-resistant Staphylococcus aureus (MRSA) in invasive isolates from southern and eastern Mediterranean countries. J Antimicrob Chemother, 2007. 60(6): p. 1310 5.

https://doi.org/10.1093/jac/dkm365

4. Yousef SA, Mahmoud SY, and E. MT., PREVALENCE OF METHICILLIN-RESISTANT STAPHYLOCOCCUS AUREUS IN SAUDI ARABIA: SYSTEMIC REVIEW AND META-ANALYSIS. 2013: Afr J Cln Exper Microbiol. p. 146-54.

5. "Middle East \& North Africa | Data". Data.Worldbank.Org , 2021, https://data.worldbank.org/country/ZQ.

6. Udo, E.E., D. Panigrahi, and A.E. Jamsheer, Molecular typing of methicillin-resistant Staphylococcus aureus isolated in a Bahrain hospital. Med Princ Pract, 2008. 17(4): p. 308-14 https://doi.org/10.1159/000129611

7. Alfouzan, W., et al., Molecular Characterization of Methicillin- Resistant Staphylococcus aureus in a Tertiary Care hospital in Kuwait. Sci Rep, 2019. 9(1): p. 18527. https://doi.org/10.1038/s41598-019-54794-8

8. Monecke, S., et al., A field guide to pandemic, epidemic and sporadic clones of methicillin-resistant Staphylococcus aureus. PLoS One, 2011. 6(4): p. e17936. https://doi.org/10.1371/journal.pone.0017936

9. Liu, G.Y., Molecular pathogenesis of Staphylococcus aureus infection Pediatr Res, 2009. 65(5 Pt 2): p. 71R-77R. have a heterogeneous lineage despite belonging to the same Spa-clonal complex. ${ }^{81}$ These findings raise the possibility that local strains might have polyphyletic origins.

Our review provides a comprehensive summary of the available literature on the epidemiology of MRSA in the MENA. Multiple important points were highlighted. Nonetheless, our work is not without limitations. Most studies in our review were single-centered reports, limiting our ability to uncover the full burden of MRSA. Furthermore, there was significant heterogeneity in molecular typing methods and reporting of results, which hinders our ability to compare between different centers or countries in the region. Furthermore, the bulk of studies did not distinguish between CO-MRSA and HOMRSA based on epidemiological criteria, and so we are unable to accurately distinguish the epidemiology of MRSA in the community vs. healthcare setting.

Conclusion. This manuscript provides a review of the current literature on MRSA epidemiology in Arab countries. There is enough evidence to suggest that the Arab region struggles from a heavy MRSA burden. Genotypes associated with European CA-MRSA play the biggest role, but Brazilian/Hungarian HA-MRSA and UK-EMRSA-15 HA-MRSA are also prevalent. Therefore, the Arab countries need to implement a nationwide surveillance system to understand better the true epidemiology and burden of MRSA in this part of the world.
https://doi.org/10.1203/PDR.0b013e31819dc44d

10. Lina, G., et al., Involvement of Panton-Valentine leukocidin-producing Staphylococcus aureus in primary skin infections and pneumonia. Clin Infect Dis, 1999. 29(5): p. 1128-32. https://doi.org/10.1086/313461

11. El-Mahdy, T.S., M. El-Ahmady, and R.V. Goering, Molecular characterization of methicillin-resistant Staphylococcus aureus isolated over a 2-year period in a Qatari hospital from multinational patients. Clin Microbiol Infect, 2014. 20(2): p. 169-73. https://doi.org/10.1111/1469-0691.12240

12. Abou Shady, H.M., et al., Staphylococcus aureus nasal carriage among outpatients attending primary health care centers: a comparative study of two cities in Saudi Arabia and Egypt. Brazilian Journal of Infectious Diseases, 2015. 19(1): p. 68-76. https://doi.org/10.1016/j.bjid.2014.09.005

13. Panhotra, B.R., A.K. Saxena, and A.S. Al Mulhim, Prevalence of methicillin-resistant and methicillin-sensitive Staphylococcus aureus nasal colonization among patients at the time of admission to the hospital. Ann Saudi Med, 2005. 25(4): p. 304-8. https://doi.org/10.5144/0256-4947.2005.304

14. Alhussaini, M.S., Methicillin-resistant Staphylococcus aureus Nasal Carriage Among Patients Admitted at Shaqra General Hospital in Saudi Arabia. Pak J Biol Sci, 2016. 19(5): p. 233-238. https://doi.org/10.3923/pjbs.2016.233.238

15. Iyer, A., et al., High incidence rate of methicillin-resistant Staphylococcus aureus (MRSA) among healthcare workers in Saudi Arabia. J Infect Dev Ctries, 2014. 8(3): p. 372-8. https://doi.org/10.3855/jidc. 3589

16. El-Mahdy, T.S., et al. Complex Clonal Diversity of Staphylococcus aureus Nasal Colonization among Community Personnel, Healthcare Workers, and Clinical Students in the Eastern Province, Saudi Arabia. Biomed Res Int, 2018. 2018: p. 4208762. 


\section{https://doi.org/10.1155/2018/4208762}

17. Zakai, S.A., Prevalence of methicillin-resistant Staphylococcus aureus nasal colonization among medical students in Jeddah, Saudi Arabia. Saud Med J, 2015. 36(7): p. 807-12. https://doi.org/10.15537/smj.2015.7.11609

18. Ghanem, H.M., A.M. Abou-Alia, and S.A. Alsirafy, Prevalence of methicillin-resistant Staphylococcus aureus colonization and infection in hospitalized palliative care patients with cancer. Am J Hosp Palliat Care, 2013. 30(4): p. 377-9 https://doi.org/10.1177/1049909112452335

19. Al-Humaidan, O.S., T.A. El-Kersh, and R.A. Al-Akeel, Risk factors of nasal carriage of Staphylococcus aureus and methicillin-resistant Staphylococcus aureus among health care staff in a teaching hospital in central Saudi Arabia. Saudi Med J, 2015. 36(9): p. 1084-90. https://doi.org/10.15537/smj.2015.9.12460

20. Alfouzan, W., R. Dhar, and E. Udo, Genetic Lineages of MethicillinResistant Staphylococcus aureus Acquired during Admission to an Intensive Care Unit of a General Hospital. Med Princ Pract, 2017. 26(2) p. 113-117. https://doi.org/10.1159/000453268

21. Pathare, N.A., et al., Comparison of Methicillin Resistant Staphylococcu Aureus in Healthy Community Hospital Visitors [CA-MRSA] and Hospital Staff [HA-MRSA]. Mediterr J Hematol Infect Dis, 2015. 7(1): p. e2015053. https://doi.org/10.4084/mjhid.2015.053

22. Pathare, N.A., et al., Prevalence of methicillin resistant Staphylococcus aureus [MRSA] colonization or carriage among healthcare workers. J Infect Public Health, 2016. 9(5): p. 571-6 https://doi.org/10.1016/j.jiph.2015.12.004

23. Biber, A., et al., A typical hospital-acquired methicillin-resistan Staphylococcus aureus clone is widespread in the community in the Gaza strip. PLoS One, 2012. 7(8): p. e42864. https://doi.org/10.1371/journal.pone.0042864

24. Kaibni, M.H., et al., Community-acquired meticillin-resistan Staphylococcus aureus in Palestine. J Med Microbiol, 2009. 58(Pt 5): p 644-7.

https://doi.org/10.1099/jmm.0.007617-0

25. El Aila, N.A., N.A. Al Laham, and B.M. Ayesh, Nasal carriage of methicillin resistant Staphylococcus aureus among health care workers at Al Shifa hospital in Gaza Strip. Bmc Infectious Diseases, 2017. 17. https://doi.org/10.1186/s12879-016-2139-1

26. Al-Bakri, A.G., et al., The epidemiology and molecular characterization of methicillin-resistant staphylococci sampled from a healthy Jordanian population. Epidemiol Infect, 2013. 141(11): p. 2384-91. https://doi.org/10.1017/S0950268813000010

27. Aqel, A.A., et al., Molecular epidemiology of nasal isolates of methicillinresistant Staphylococcus aureus from Jordan. J Infect Public Health, 2015. 8(1): p. $90-7$. https://doi.org/10.1016/j.jiph.2014.05.007

28. Sfeir, M., et al., Prevalence of Staphylococcus aureus methicillinsensitive and methicillin-resistant nasal and pharyngeal colonization in outpatients in Lebanon. Am J Infect Control, 2014. 42(2): p. 160-3. https://doi.org/10.1016/j.ajic.2013.08.008

29. Halablab, M.A., et al., Staphylococcus aureus nasal carriage rate and associated risk factors in individuals in the community. Epidemiol Infect, 2010. 138(5): p. 702-6. https://doi.org/10.1017/S0950268809991233

30. Hussein, N.R., M.S. Assafi, and T. Ijaz, Methicillin-resistant Staphylococcus aureus nasal colonisation amongst healthcare workers in Kurdistan Region, Iraq. J Glob Antimicrob Resist, 2017. 9: p. 78-81. https://doi.org/10.1016/j.jgar.2017.01.010

31. Khairalla, A.S., R. Wasfi, and H.M. Ashour, Carriage frequency, phenotypic, and genotypic characteristics of methicillin-resistant Staphylococcus aureus isolated from dental healthcare personnel, patients, and environment. Sci Rep, 2017. 7(1): p. 7390. https://doi.org/10.1038/s41598-017-07713-8

32. Abaza, A.F., et al., Nasal carriage of methicillin-resistant Staphylococcus aureus and the effect of tea extracts on isolates. J Egypt Public Health Assoc, 2016. 91(3): p. 135-143. https://doi.org/10.1097/01.EPX.0000491266.22392.c3

33. Hefzy, E.M., G.M. Hassan, and F. Abd El Reheem, Detection of PantonValentine Leukocidin-Positive Methicillin-Resistant Staphylococcus aureus Nasal Carriage among Egyptian Health Care Workers. Surg Infect (Larchmt), 2016. 17(3): p. 369-75. https://doi.org/10.1089/sur.2015.192

34. Djoudi, F., et al., Descriptive epidemiology of nasal carriage of Staphylococcus aureus and methicillin-resistant Staphylococcus aureus among patients admitted to two healthcare facilities in Algeria. Microb Drug Resist, 2015. 21(2): p. 218-23. https://doi.org/10.1089/mdr.2014.0156

35. Antri, K., et al., High levels of Staphylococcus aureus and MRSA carriage in healthy population of Algiers revealed by additional enrichment and multisite screening. Eur J Clin Microbiol Infect Dis, 2018. 37(8): p. 15211529

https://doi.org/10.1007/s10096-018-3279-6

36. Ben Slama, K., et al., Nasal carriage of Staphylococcus aureus in healthy humans with different levels of contact with animals in Tunisia: genetic lineages, methicillin resistance, and virulence factors. Eur J Clin Microbiol Infect Dis, 2011. 30(4): p. 499-508. https://doi.org/10.1007/s10096-010-1109-6

37. Eed, E.M., et al., Molecular characterisation of Panton-Valentine leucocidin-producing methicillin-resistant Staphylococcus aureus clones isolated from the main hospitals in Taif, KSA. Indian J Med Microbiol, 2016. 34(4): p. 476-482 https://doi.org/10.4103/0255-0857.195364

38. Asghar, A.H., Molecular characterization of methicillin-resistant Staphylococcus aureus isolated from tertiary care hospitals. Pak J Med Sci, 2014. 30(4): p. 698-702. https://doi.org/10.12669/pjms.304.4946

39. Haseeb, A., et al., Antimicrobial resistance among pilgrims: a retrospective study from two hospitals in Makkah, Saudi Arabia. Int J Infect Dis, 2016. 47: p. 92-4. https://doi.org/10.1016/j.ijid.2016.06.006

40. Abdel-Fattah, M.M., Surveillance of nosocomial infections at a Saudi Arabian military hospital for a one-year period. Ger Med Sci, 2005. 3: p. Doc06.

41. Al Jalaf, M., et al., Methicillin resistant Staphylococcus Aureus in emergency department patients in the United Arab Emirates. BMC Emerg Med, 2018. 18(1): p. 12 https://doi.org/10.1186/s12873-018-0164-7

42. Al-Yaqoubi, M. and K. Elhag, Susceptibilities of common bacterial isolates from oman to old and new antibiotics. Oman Med J, 2008. 23(3): p. 173-8.

43. Al Rahmany, D., et al., Exploring bacterial resistance in Northern Oman, a foundation for implementing evidence-based antimicrobial stewardship program. Int J Infect Dis, 2019. 83: p. 77-82. https://doi.org/10.1016/j.ijid.2019.04.004

44. Khan, F.Y., et al., Epidemiology of bacteraemia in Hamad general hospital, Qatar: a one year hospital-based study. Travel Med Infect Dis, 2010. 8(6): p. 377-87. https://doi.org/10.1016/j.tmaid.2010.10.004

45. Al Laham, N., et al., MRSA clonal complex 22 strains harboring toxic shock syndrome toxin (TSST-1) are endemic in the primary hospital in Gaza, Palestine. PLoS One, 2015. 10(3): p. e0120008. https://doi.org/10.1371/journal.pone.0120008

46. Al-Zoubi, M.S., et al., Antimicrobial susceptibility pattern of Staphylococcus aureus isolated from clinical specimens in Northern area of Jordan. Iran J Microbiol, 2015. 7(5): p. 265-72.

47. Bazzoun, D.A., et al., Molecular typing of Staphylococcus aureus collected from a Major Hospital in Amman, Jordan. J Infect Dev Ctries, 2014. 8(4): p. 441-7. https://doi.org/10.3855/jidc. 3676

48. Tokajian, ST, et al., Molecular characterization of Staphylococcus aureus in Lebanon. Epidemiol Infect, 2010. 138(5): p. 707-12. https://doi.org/10.1017/S0950268810000440

49. Chamoun, K., et al., Surveillance of antimicrobial resistance in Lebanese hospitals: retrospective nationwide compiled data. Int J Infect Dis, 2016. 46: p. 64-70. https://doi.org/10.1016/j.ijid.2016.03.010

50. Araj, G.F., et al., A reflection on bacterial resistance to antimicrobial agents at a major tertiary care center in Lebanon over a decade. J Med Liban, 2012. 60(3): p. 125-35.

51. Co, E.M., E.F. Keen, 3rd, and W.K. Aldous, Prevalence of methicillinresistant staphylococcus aureus in a combat support hospital in Iraq. Mil Med, 2011. 176(1): p. 89-93. https://doi.org/10.7205/MILMED-D-09-00126

52. Roberts, S.S. and R.J. Kazragis, Methicillin-resistant Staphylococcus aureus infections in U.S. service members deployed to Iraq. Mil Med, 2009. 174(4): p. 408-11. https://doi.org/10.7205/MILMED-D-02-8408

53. Babakir-Mina, M., et al., Antibiotic susceptibility of vancomyin and nitrofurantoin in Staphylococcus aureus isolated from burnt patients in Sulaimaniyah, Iraqi Kurdistan. New Microbiol, 2012. 35(4): p. 439-46.

54. Abd El-Hamid, M.I. and M.M. Bendary, Comparative phenotypic and genotypic discrimination of methicillin resistant and susceptible 
Staphylococcus aureus in Egypt. Cell Mol Biol (Noisy-le-grand), 2015. 61(4): p. 101-12.

55. Bendary, M.M., et al., Characterization of Methicillin Resistant Staphylococcus aureus isolated from human and animal samples in Egypt Cell Mol Biol (Noisy-le-grand), 2016. 62(2): p. 94-100.

56. Sobhy, N., et al., Community-acquired methicillin-resistant Staphylococcus aureus from skin and soft tissue infections (in a sample of Egyptian population): analysis of mec gene and staphylococcal cassette chromosome. Braz J Infect Dis, 2012. 16(5): p. 426-31. https://doi.org/10.1016/j.bjid.2012.08.004

57. Abdel-Maksoud, M., et al., Methicillin-Resistant Staphylococcus aureus Recovered from Healthcare- and Community-Associated Infections in Egypt. Int J Bacteriol, 2016. 2016: p. 5751785. https://doi.org/10.1155/2016/5751785

58. Alioua, M.A., et al., Emergence of the European ST80 clone of community-associated methicillin-resistant Staphylococcus aureus as a cause of healthcare-associated infections in Eastern Algeria. Med Mal Infect, 2014. 44(4): p. 180-3. https://doi.org/10.1016/j.medmal.2014.01.006

59. Djoudi, F., et al., Panton-Valentine leukocidin positive sequence type 80 methicillin-resistant Staphylococcus aureus carrying a staphylococcal cassette chromosome mec type IVc is dominant in neonates and children in an Algiers hospital. New Microbiol, 2013. 36(1): p. 49-55.

60. Djahmi, N., et al., Molecular epidemiology of Staphylococcus aureus strains isolated from inpatients with infected diabetic foot ulcers in an Algerian University Hospital. Clin Microbiol Infect, 2013. 19(9): p. E398-404. https://doi.org/10.1111/1469-0691.12199

61. Antri, K., et al., High prevalence of methicillin-resistant Staphylococcus aureus clone ST80-IV in hospital and community settings in Algiers. Clin Microbiol Infect, 2011. 17(4): p. 526-32. https://doi.org/10.1111/j.1469-0691.2010.03273.x

62. Rebiahi, S.A., et al., Emergence of vancomycin-resistant Staphylococcus aureus identified in the Tlemcen university hospital (North-West Algeria) Med Mal Infect, 2011. 41(12): p. 646-51. https://doi.org/10.1016/j.medmal.2011.09.010

63. Bouchami, O., W. Achour, and A. Ben Hassen, Typing of staphylococcal cassette chromosome mec encoding methicillin resistance in Staphylococcus aureus strains isolated at the bone marrow transplant centre of Tunisia. Curr Microbiol, 2009. 59(4): p. 380-5. https://doi.org/10.1007/s00284-009-9448-1

64. Mesrati, I., et al., Clinical isolates of Pantone-Valentine leucocidin- and gamma-haemolysin-producing Staphylococcus aureus: prevalence and association with clinical infections. J Hosp Infect, 2010. 75(4): p. 265-8. https://doi.org/10.1016/j.jhin.2010.03.015

65. Elhani, D., et al., Clonal lineages detected amongst tetracycline-resistant meticillin-resistant Staphylococcus aureus isolates of a Tunisian hospital, with detection of lineage ST398. J Med Microbiol, 2015. 64(6): p. 623-9. https://doi.org/10.1099/jmm.0.000066

66. Alreshidi, M.A., et al., Genetic variation among methicillin-resistan Staphylococcus aureus isolates from cancer patients in Saudi Arabia. Eur J Clin Microbiol Infect Dis, 2013. 32(6): p. 755-61. https://doi.org/10.1007/s10096-012-1801-9

67. Monecke, S., et al., Characterisation of MRSA strains isolated from patients in a hospital in Riyadh, Kingdom of Saudi Arabia. BMC Microbiol, 2012. 12: p. 146. https://doi.org/10.1186/1471-2180-12-146

68. Senok, A., et al., Molecular characterization of methicillin-resistan Staphylococcus aureus in nosocomial infections in a tertiary-care facility: emergence of new clonal complexes in Saudi Arabia. New Microbes New Infect, 2016. 14: p. 13-8. https://doi.org/10.1016/j.nmni.2016.07.009

69. Boswihi, S.S., E.E. Udo, and N. Al-Sweih, Shifts in the Clonal Distribution of Methicillin-Resistant Staphylococcus aureus in Kuwait Hospitals: 1992-2010. PLoS One, 2016. 11(9): p. e0162744. https://doi.org/10.1371/journal.pone.0162744

70. Udo, E.E., et al., Genetic lineages of community-associated methicillinresistant Staphylococcus aureus in Kuwait hospitals. J Clin Microbiol, 2008. 46(10): p. 3514-6.
https://doi.org/10.1128/JCM.00966-08

71. Sonnevend Á, B.I., Alkaabi M, Jumaa P, Al Haj M, Ghazawi A, Akawi $\mathrm{N}$, Jouhar FS, Hamadeh MB, Pál T., Change in meticillin-resistant Staphylococcus aureus clones at a tertiary care hospital in the United Arab Emirates over a 5-year period. J Clin Pathol. , 2012. 65(2): p. 178-82. https://doi.org/10.1136/jclinpath-2011-200436

72. Moussa, I., et al., A novel multiplex PCR for molecular characterization of methicillin resistant Staphylococcus aureus recovered from Jeddah, Kingdom of Saudi Arabia. Indian J Med Microbiol, 2012. 30(3): p. 296301. https://doi.org/10.4103/0255-0857.99490

73. Boswihi, S.S., et al., Emerging variants of methicillin-resistant Staphylococcus aureus genotypes in Kuwait hospitals. PLoS One, 2018 13(4): p. e0195933. https://doi.org/10.1371/journal.pone.0195933

74. Huang, X.Z., et al., Methicillin-resistant Staphylococcus aureus infection in combat support hospitals in three regions of Iraq. Epidemiol Infect, 2011. 139(7): p. 994-7. https://doi.org/10.1017/S0950268810001950

75. Basset, P., W. Amhis, and D.S. Blanc, Changing molecular epidemiology of methicillin-resistant Staphylococcus aureus in an Algerian hospital. J Infect Dev Ctries, 2015. 9(2): p. 206-9. https://doi.org/10.3855/jidc.4620

76. Ouchenane, Z., et al., Molecular characterization of methicillin-resistant Staphylococcus aureus isolates in Algeria. Pathol Biol (Paris), 2011. 59(6): p. e129-32.

77. Mariem, B.J., et al., Molecular characterization of methicillin-resistant Panton-valentine leukocidin positive staphylococcus aureus clones disseminating in Tunisian hospitals and in the community. BMC Microbiol, 2013. 13: p. 2. https://doi.org/10.1186/1471-2180-13-2

78. Udo, E.E., S.S. Boswihi, and N. Al-Sweih, High prevalence of toxic shock syndrome toxin-producing epidemic methicillin-resistant Staphylococcus aureus 15 (EMRSA-15) strains in Kuwait hospitals. New Microbes New Infect, 2016. 12: p. 24-30 https://doi.org/10.1016/j.nmni.2016.03.008

79. Harastani, H.H., G.F. Araj, and ST Tokajian, Molecular characteristics of Staphylococcus aureus isolated from a major hospital in Lebanon. Int J Infect Dis, 2014. 19: p. 33-8. https://doi.org/10.1016/j.ijid.2013.10.007

80. Senok, A., et al., Diversity of methicillin-resistant Staphylococcus aureus CC22-MRSA-IV from Saudi Arabia and the Gulf region. Int J Infect Dis, 2016. 51: p. 31-35. https://doi.org/10.1016/j.ijid.2016.08.016

81. Harastani, H.H. and ST Tokajian, Community-associated methicillinresistant Staphylococcus aureus clonal complex 80 type IV (CC80MRSA-IV) isolated from the Middle East: a heterogeneous expanding clonal lineage. PLoS One, 2014. 9(7): p. e103715. https://doi.org/10.1371/journal.pone. 0103715

82. Udo, E.E. and E. Sarkhoo, The dissemination of ST80-SCCmec-IV community-associated methicillin resistant Staphylococcus aureus clone in Kuwait hospitals. Ann Clin Microbiol Antimicrob, 2010. 9: p. 31. https://doi.org/10.1186/1476-0711-9-31

83. Moussa, I. and A.M. Shibl, Molecular characterization of methicillinresistant Staphylococcus aureus recovered from outpatient clinics in Riyadh, Saudi Arabia. Saudi Med J, 2009. 30(5): p. 611-7.

84. Udo, E.E. and S.S. Boswihi, Antibiotic Resistance Trends in MethicillinResistant Staphylococcus aureus Isolated in Kuwait Hospitals: 2011-2015. Med Princ Pract, 2017. 26(5): p. 485-490. https://doi.org/10.1159/000481944

85. Baddour, M.M., M.M. Abuelkheir, and A.J. Fatani, Trends in antibiotic susceptibility patterns and epidemiology of MRSA isolates from several hospitals in Riyadh, Saudi Arabia. Ann Clin Microbiol Antimicrob, 2006. 5: p. 30.

https://doi.org/10.1186/1476-0711-5-30

86. Tiemersma, E.W., et al., Methicillin-resistant Staphylococcus aureus in Europe, 1999-2002. Emerg Infect Dis, 2004. 10(9): p. 1627-34. https://doi.org/10.3201/eid1009.040069 


\section{Supplementary Files}

Supplementary Table 1. The table presents the relative global frequencies of spa types. Only those $>/=0.5 \%$ are reported here. Data is adopted from http://www.spaserver.ridom.de. It also lists the Arab countries that reported these spa types based on our review of literature.

\begin{tabular}{|c|c|c|}
\hline \multicolumn{3}{|c|}{ Common Globally Reported spa Types } \\
\hline Spa type & Relative global frequency & Arab countries reporting this spa type \\
\hline t032 & $9.98 \%$ & $\mathrm{KSA}, \mathrm{Ku}, \mathrm{Leb}, \mathrm{UAE}$ \\
\hline t003 & $8.57 \%$ & $\mathrm{Ku}$ \\
\hline t002 & $6.56 \%$ & $\mathrm{KSA}, \mathrm{Ku}, \mathrm{Qa}, \mathrm{UAE}$ \\
\hline t008 & $5.81 \%$ & Egy, Gaz, KSA, Ku, Leb, Qa \\
\hline t011 & $3.30 \%$ & \\
\hline t127 & $2.64 \%$ & $\mathrm{Ku}, \mathrm{UAE}$ \\
\hline t034 & $2.18 \%$ & \\
\hline t084 & $1.75 \%$ & Egy, Ku, KSA \\
\hline t012 & $1.53 \%$ & Jor \\
\hline t037 & $1.12 \%$ & Alg, Gaz, Jor, KSA, Ku, Leb, Tun, UAE \\
\hline t021 & $1.07 \%$ & Egy, Jor, Ku, Leb \\
\hline t015 & $1.02 \%$ & \\
\hline t091 & $0.99 \%$ & Jor \\
\hline t044 & $0.85 \%$ & Alg, Egy, Gaz, Jor, KSA, Ku, Leb, Qa, Tun, UAE \\
\hline $\mathrm{t} 022$ & $0.79 \%$ & \\
\hline $\mathrm{t} 437$ & $0.78 \%$ & \\
\hline t005 & $0.70 \%$ & $\mathrm{Alg}, \mathrm{Ku}, \mathrm{UAE}$ \\
\hline t045 & $0.69 \%$ & $\mathrm{Ku}$ \\
\hline t067 & $0.66 \%$ & \\
\hline $\mathrm{t} 024$ & $0.64 \%$ & \\
\hline t223 & $0.62 \%$ & Alg, Egy, Gaz, Jor, KSA, Ku \\
\hline t189 & $0.59 \%$ & KSA \\
\hline t304 & $0.58 \%$ & $\mathrm{KSA}, \mathrm{Ku}, \mathrm{Leb}, \mathrm{UAE}$ \\
\hline t018 & $0.56 \%$ & Jor, Ku, UAE \\
\hline t004 & $0.51 \%$ & \\
\hline t026 & $0.51 \%$ & \\
\hline
\end{tabular}

Alg: Algeria; Egy: Egypt; Gaz: Gaza; Jor: Jordan; KSA: kingdom of Saudi Arabia; Ku: Kuwait; Leb: Lebanon; Qa: Qatar; Tun: Tunisia; UAE: United Arab Emirates 
Supplementary Table 2. Reported Rates of Methicillin-Resistance among Staphylococcus aureus from clinical specimen in MENA Region.

MRSA rates presented as total MRSA/total S. aureus isolates. All isolates were from various clinical samples unless otherwise specified.

Numerical values are rounded to the nearest whole digit.

\begin{tabular}{|c|c|c|c|}
\hline Ref & \# S. aureus isolate & \# MRSA (\%) & Study site (year) \\
\hline \multicolumn{4}{|r|}{ KSA } \\
\hline [87] & $\begin{array}{l}\text { S. aureus from clinical } \\
\text { samples: } \\
\text { Year } 1999 \\
\text { Year 2002 } \\
\text { Year 2003 }\end{array}$ & $\begin{array}{c}(6 \%) \\
(2 \%) \\
(10 \%) \\
(8 \%)\end{array}$ & Dhahran Health Center, Dhahran (1999-2003) \\
\hline [37] & 445 & $71(16 \%)$ & Multiple hospitals ( $\mathrm{n}=\mathrm{NS})$, Taif city (2013-15) \\
\hline [38] & 206 & $114(55 \%)$ & Multiple hospitals (n=5), Makkah city (2012) \\
\hline [39] & 57 & $36(63 \%)$ & Multiple hospitals ( $\mathrm{n}=2$ ), Makkah city (2015) \\
\hline [40] & 252 & $146(58 \%)$ & Al-Hada Armed Forces Hospital, Taif city (2004) \\
\hline \multicolumn{4}{|r|}{ UAE } \\
\hline [71] & $\begin{array}{l}701 \\
490 \text { Year } 2003 \\
211 \text { Year } 2008 \\
\end{array}$ & $\begin{array}{c}52(7 \%) \\
26(5 \%) \\
26(12 \%) \\
\end{array}$ & Tawam Hospital, Al Ain (2003-8) \\
\hline [41] & $62^{\mathrm{A}}$ & $18(29 \%)$ & Rashid Hospital and Trauma Center, Dubai (2011-12) \\
\hline \multicolumn{4}{|r|}{ Oman } \\
\hline [42] & 29 & $4(14 \%)$ & Royal Hospital, Muscat (2007) \\
\hline [43] & 1972 & $733(37 \%)$ & Sohar Hospital, Sohar (2016-17) \\
\hline \multicolumn{4}{|r|}{ Qatar } \\
\hline [44] & $53^{\mathrm{B}}$ & $7(13 \%)$ & Hamad General Hospital, Doha (2007-8) \\
\hline [11] & 840 & $176(21 \%)$ & Al-Ahli Hospital, Doha (2009-10) \\
\hline \multicolumn{4}{|r|}{ Gaza } \\
\hline [45] & $\begin{array}{l}215 \\
126 \text { Year } 2008 \\
89 \text { Year } 2012 \\
\end{array}$ & $\begin{array}{l}121(56 \%) \\
69(55 \%) \\
52(58 \%) \\
\end{array}$ & Al-Shifa Hospital (2008-12) \\
\hline \multicolumn{4}{|r|}{ Jordan } \\
\hline [46] & 358 & $113(32 \%)$ & Multiple hospitals ( $\mathrm{n}=\mathrm{NS}$ ), Northern Jordan. (2008-12) \\
\hline [47] & 60 & $41(68 \%)$ & Jordan University Hospital, Amman (2009-10) \\
\hline \multicolumn{4}{|r|}{ Lebanon } \\
\hline [48] & $130^{\mathrm{C}}$ & $93(72 \%)$ & American University of Beirut Medical Center, Beirut (2006-7) \\
\hline [49] & $4890^{\mathrm{D}}$ & $1350(28 \%)$ & $\begin{array}{l}\text { Multiple hospitals ( } \mathrm{n}=16) \text {, Beirut, Mount Lebanon, North Lebanon, and South } \\
\text { Lebanon }\end{array}$ \\
\hline [50] & $\begin{array}{l}1167 \\
378 \text { Year 2000-1 } \\
310 \text { Year 2005-6 } \\
479 \text { Year 2010-11 }\end{array}$ & $\begin{array}{l}282(24 \%) \\
124(33 \%) \\
62(20 \%) \\
96(20 \%)\end{array}$ & American University of Beirut Medical Center, Beirut (2000-11) \\
\hline \multicolumn{4}{|r|}{ Iraq } \\
\hline [51] & $505^{\mathbf{E}}$ & $251(50 \%)$ & Ibn Sina Hospital, Baghdad (2005-9) \\
\hline [51] & 152 & $52(34 \%)$ & Ibn Sina Hospital, Baghdad (2005-9) \\
\hline [52] & $22^{\mathrm{F}}$ & $15(68 \%)$ & Witmer Troop Medical Clinic, Camp Liberty, Baghdad (2008) \\
\hline [53] & $654^{\mathbf{G}}$ & $576(88 \%)$ & Sulaymaniyah Burns and Plastic Hospital, Sulaymaniyah, Kurdistan region (2008-11) \\
\hline \multicolumn{4}{|r|}{ Egypt } \\
\hline [54] & 55 & $18(33 \%)$ & University Hospital and Diagnostic Laboratories, Zagazig, and Assuit (2010-12) \\
\hline [55] & 64 & $38(59 \%)$ & Suez Canal University, Ismailia (2013) \\
\hline [56] & $38^{\mathbf{H}}$ & $18(47 \%)$ & Alexandria University, Alexandria (NR) \\
\hline [57] & 631 & $364(58 \%)$ & Multiple hospitals $(\mathrm{n}=12)$ \\
\hline \multicolumn{4}{|r|}{ Algeria } \\
\hline [58] & 148 & $92(62 \%)$ & Multiple hospitals (n=4), Annaba (2010) \\
\hline [59] & 129 & $25(19 \%)$ & Beni Messous University Hospital, Algiers (2010-11) \\
\hline [60] & $85^{\mathbf{I}}$ & $73(86 \%)$ & University Hospital Ibn Rochd, Annaba (2011-12) \\
\hline [61] & 221 & $99(44 \%)$ & Mustapha Bacha University Hospital, Algiers (2006-7) \\
\hline [62] & $220^{\mathrm{J}}$ & $165(75 \%)$ & Tlemecen teaching hospital, Tlemecen (2007-9) \\
\hline \multicolumn{4}{|r|}{ Tunisia } \\
\hline [63] & $72^{\mathrm{K}}$ & $13(18 \%)$ & Centre National de Greffe de Moelle Osseuse, Laboratory Service, Tunis (1998-2007) \\
\hline [64] & 143 & $58(40 \%)$ & Charles Nicolle Hospital of Tunis, Tunis (2005-7) \\
\hline [65] & 387 & $99(26 \%)$ & Military Hospital of Tunis, Tunis (2011-12) \\
\hline
\end{tabular}


${ }^{\text {A }}$ Purulent skin and soft tissue infection, ${ }^{\mathbf{B}}$ Bacteremia, ${ }^{\mathbf{C}}$ Random wound and respiratory infections, ${ }^{\mathbf{D}}$ Study sites represent $40.7 \%$ of all hospital, eds in Lebanon at time of study, ${ }^{\mathbf{E}}$ US military personnel in combat support hospital, ${ }^{\mathbf{F}} 70 \%$ of the patients were active-duty service members; the rest were a mix of U.S. Department of Defense civilians and contractors and third country nationals (including India, Pakistan, and Turkey), ${ }^{\mathbf{G}}$ Burn patients, ${ }^{\mathbf{H}}$ Skin and soft tissue infection (does not specify if purulent vs non-purulent), ${ }^{\mathbf{I}}$ Diabetic foot infection, ${ }^{\mathbf{J}}$ Surgical wound infection $>48$ hours postoperatively, ${ }^{K}$ Neutropenic patients at bone marrow transplant center. 


\begin{tabular}{|c|c|c|c|c|}
\hline Ref & Description & \multicolumn{2}{|c|}{ Molecular typing (CC, Spa, MLST, PFGE) } & Study location (year) \\
\hline \multicolumn{5}{|c|}{ KSA } \\
\hline [12] & $\begin{array}{l}26 \text { MRSA from nasal swabs of } \\
\text { outpatients }^{\mathrm{A}}\end{array}$ & $\begin{array}{l}\text { CC15 (27\%): } \\
\text { [PVL-] CC15-IVa-t084 (7\%) } \\
\text { [PVL-] CC15-V-t084 (7\%) } \\
\text { [PVL-] CC15-V-t085 (13\%) } \\
\text { CC8 (13\%): } \\
\text { [PVL-] CC8-V-t008 }(13 \%) \\
\text { CC25 (13\%): } \\
\text { [PVL-] CC25-V-t349 (7\%) } \\
\text { [PVL-] CC25-IVa-t660 (7\%) } \\
\text { CC5 (7\%): } \\
\text { [PVL-] CC5-V-t002 (6.7\%) }\end{array}$ & $\begin{array}{l}\text { CC22 (7\%): } \\
\text { [PVL-] CC22-V-t223 (6.7\%) } \\
\text { CC80 (7\%): } \\
\text { [PVL+] CC80-NT-t376 (6.7\%) } \\
\text { CC188 (7\%): } \\
\text { [PVL-] CC188-V-t1 } 89(6.7 \%) \\
\text { CC-NA (20\%): } \\
\text { [PVL-] IVa-t442 }(6.7 \%) \\
\text { [PVL+] NT-t6836 (6.7\%) } \\
\text { [PVL+] IVa-t1 1841 (6.7\%) }\end{array}$ & $\begin{array}{l}\text { Outpatient clinics, Buraydah city } \\
\text { (2011) }\end{array}$ \\
\hline [16] & $\begin{array}{l}20 \text { MRSA from nasal swabs of } \\
\text { healthy volunteers, clinical } \\
\text { students, HCWs }\end{array}$ & $\begin{array}{l}\text { CC80 (20\%): } \\
\text { CC80-ST80-IV-t131 (20\%) } \\
\text { CC22 (20\%): } \\
\text { CC22-IV-t1328 (20\%) } \\
\text { CC5 (20\%): } \\
\text { CC5-IV-t688 (20\%) }\end{array}$ & $\begin{array}{l}\text { PFGE F (20\%): } \\
\text { PFGE F-ST6-II-t304 (20\%) } \\
\text { CC1 (10\%): } \\
\text { CC1-IVa-t128 (10\%) } \\
\text { PFGE K CC88(10\%): } \\
\text { PFGE K-CC88-IV-t1339 (10\%) }\end{array}$ & Al-Ahsa (NR) \\
\hline \multicolumn{5}{|c|}{ Kuwait } \\
\hline [20] & $\begin{array}{l}18 \text { Nasal, axillary, and groin } \\
\text { swabs from ICU inpatients }\end{array}$ & $\begin{array}{l}\text { ST239 (72\%): } \\
\text { ST239-III-t421 }(33 \%) \\
\text { ST239-III-t945 }(28 \%) \\
\text { ST239-III-t4410 (6\%) } \\
\text { ST239-III-t388 }(6 \%) \\
\text { ST22 (11\%): } \\
\text { ST22-IV-t223 }(6 \%) \\
\text { ST22-IV-t3010 (6\%) }\end{array}$ & $\begin{array}{l}\text { ST97 (11\%): } \\
\text { ST97-IV-t6665 (6\%) } \\
\text { ST97-IV-t1234 (6\%) } \\
\text { ST80 (6\%): } \\
\text { ST80-IV-t044 (6\%) }\end{array}$ & $\begin{array}{l}\text { Farwania Hospital, Kuwait City } \\
(2005-7)\end{array}$ \\
\hline \multicolumn{5}{|c|}{ Gaza } \\
\hline [23] & $\begin{array}{l}94 \text { MRSA from nasal swabs of } \\
\text { healthy children and parents }\end{array}$ & $\begin{array}{l}\text { CC22 (76\%): } \\
\text { [PVL-] ST22-IVa-t223 (64\%) } \\
\text { [PVL+] ST22-IVa-t223 (2\%) } \\
\text { [PVL-] ST22-V-t223 (7\%) } \\
\text { [PVL-] ST1784-V-t223 (2\%) } \\
\text { CC88 (7\%): } \\
\text { [PVL-] ST78-IVa (7\%) } \\
\text { CC80 (5\%): } \\
\text { [PVL+] ST80-IVa (4\%) } \\
\text { [PVL+] ST80-IV (1\%) }\end{array}$ & $\begin{array}{l}\text { CC5 (3\%): } \\
\text { [PVL-] ST5-V (1\%) } \\
\text { [PVL-] ST1785-IVa (2\%) } \\
\text { CC30 (2\%): } \\
\text { [PVL+] ST30-IVc (1\%) } \\
\text { [PVL-] ST1734-IVc (1\%) } \\
\text { CC913 (1\%): } \\
\text { [PVL-] ST913-IVa (1\%) } \\
\text { Others (5\%): } \\
\text { [PVL-] ND (5\%) }\end{array}$ & $\begin{array}{l}12 \text { Gaza neighborhoods and } \\
\text { villages, northern and central } \\
\text { Gaza (2009) }\end{array}$ \\
\hline \multicolumn{5}{|c|}{ Jordan } \\
\hline [26] & $\begin{array}{l}37 \text { MRSA from nasal and hand } \\
\text { swabs of healthy volunteers }\end{array}$ & 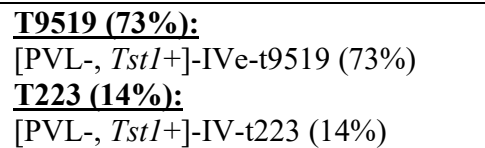 & $\begin{array}{l}\text { T044 (5\%): } \\
\text { PVL+, Tst } 1+]- \text { IVc-t044 (5\%) } \\
\text { NT (8\%): } \\
\text { [PVL-, Tst } 1+]- \text { IVe-NT (8\%) }\end{array}$ & $\begin{array}{l}\text { University of Jordan, Amman, } \\
\text { (2009) }\end{array}$ \\
\hline [27] & $\begin{array}{l}26 \text { MRSA from nasal swabs of } \\
\text { healthy volunteers }\end{array}$ & $\begin{array}{l}\text { CC22 (46\%): } \\
\text { [PVL-, Tst } 1+] \text { CC22-IVa-t223 } \\
\text { (39\%) } \\
\text { [PVL-, Tst } 1+] \text { CC22-IVa-t6397 } \\
\text { (4\%) } \\
\text { [PVL-, Tst } 1-] \text { CC22-IVa-t790 (4\%) } \\
\text { CC30 (23\%): } \\
\text { [PVL-, Tst } 1+] \text { CC30-IVc-t012 (4\%) } \\
\text { [PVL-, Tst } 1 \text {-] CC30-Ivg-t021 (4\%) } \\
\text { [PVL-, Tst } 1 \text {-] CC30-IVa-t021 (4\%) } \\
\text { [PVL-, ts1+] CC30-Ivc-t253 (4\%) } \\
\text { [PVL-, Tst } 1+] \text { CC30-Ivc-t1651 } \\
(8 \%)\end{array}$ & $\begin{array}{l}\text { CC5 (12\%): } \\
{[\text { PVL-, Tst } 1 \text {-] CC5-IVa-t214 }} \\
\text { (12\%) } \\
\text { CC1 (8\%): } \\
\text { [PVL-, Tst } 1 \text {-] CC1-IVa-t386 } \\
\text { (8\%) } \\
\text { CC80 (4\%): } \\
\text { [PVL+, Tst } 1 \text {-] CC80-IVc-t044 } \\
\text { (4\%) } \\
\text { CC97 (4\%): } \\
\text { [PVL-, Tst } 1 \text { - }] \text { CC97-NT-t1234 } \\
\text { (4\%) } \\
\text { CC15 (4\%): } \\
{[\text { PVL+, Tst } 1 \text { - }] \text { CC15-NT-t803 }} \\
(4 \%)\end{array}$ & $\begin{array}{l}\text { Al-Karak Hospital, Alkarak } \\
\text { province (2011-12) }\end{array}$ \\
\hline
\end{tabular}


30 MRSA from nasal swabs of HCWs

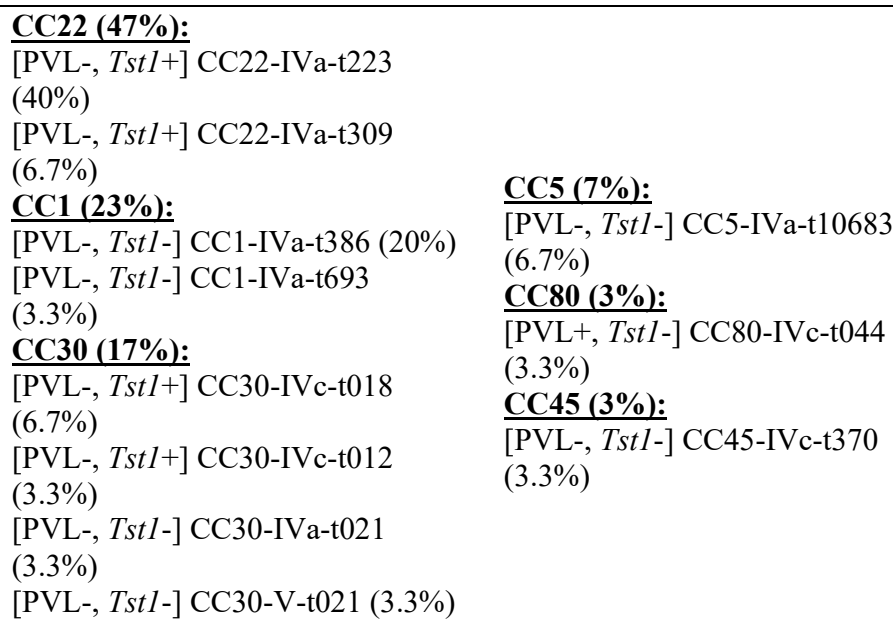

\begin{tabular}{|c|c|c|c|c|}
\hline [33] & $\begin{array}{l}30 \text { MRSA from nasal swabs of } \\
\text { HCWs }\end{array}$ & - & - & $\begin{array}{l}\text { Fayoum University Hospital, } \\
\text { Fayoum, Egypt. (NR) }\end{array}$ \\
\hline [31] & $\begin{array}{l}21 \text { MRSA from hands and } \\
\text { nasal swabs of HCWs }\end{array}$ & 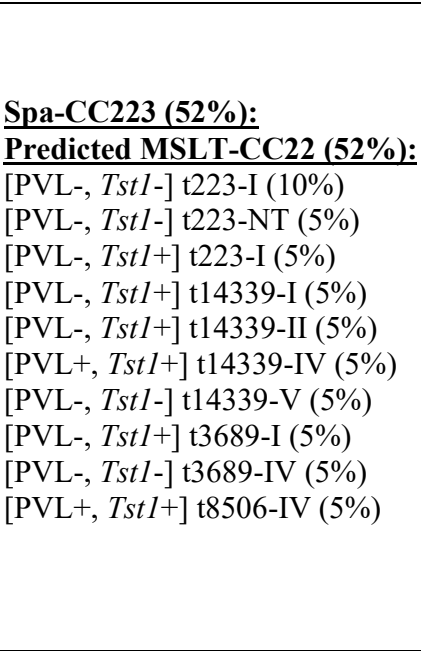 & 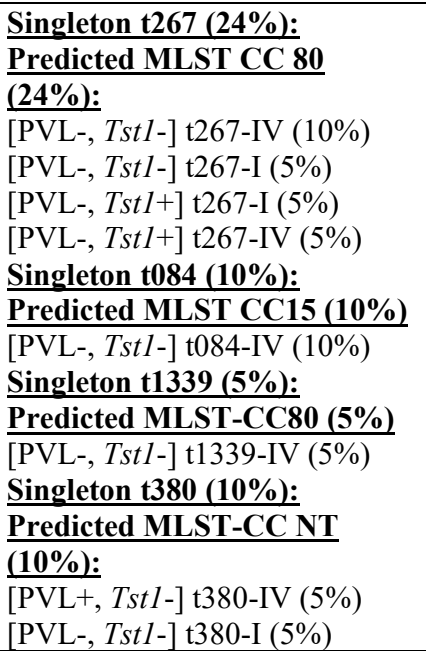 & $\begin{array}{l}\text { Beni-Suef University, Beni-Suef } \\
\text { (NR) }\end{array}$ \\
\hline [12] & $\begin{array}{l}33 \text { MRSA from nasal swabs of } \\
\text { outpatients }^{\mathbf{B}}\end{array}$ & $\begin{array}{l}\text { CC5 (30\%): } \\
\text { [PVL-] CC5-V-t688 (30\%) } \\
\text { CC30 (20\%): } \\
\text { PVL-] CC30-IVa-t021 (10\%) } \\
\text { [PVL-] CC30-IVa-t318 (10\%) } \\
\text { CC80 (15\%): } \\
{[\text { PVL+] CC } 80-\text { IVa-t044 (5\%) }} \\
{[\text { PVL+] CC } 80-V-t 044(10 \%)}\end{array}$ & $\begin{array}{l}\text { CC88 (15\%): } \\
\text { [PVL-] CC88-IVa-t786 (15\%) } \\
\text { CC22 (10\%): } \\
\text { PVL-] CC22-V-t223 (5\%) } \\
\text { [PVL-] CC22-IVa-t223 (5\%) } \\
\text { CC8 (5\%): } \\
\text { [PVL+] CC } 8-V-t 008(5 \%) \\
\text { CC-NA (5\%): } \\
\text { [PVL-] V-t11839 (5\%) }\end{array}$ & Al Kanater, Al Kheireya (2011) \\
\hline \multicolumn{5}{|c|}{$\begin{array}{rr}\text { Algeria } \\
\end{array}$} \\
\hline [34] & $\begin{array}{l}9 \text { MRSA from nasal swabs of } \\
\text { inpatients }<48 \text { hours of } \\
\text { admission }\end{array}$ & $\begin{array}{l}\text { ST80 (44\%) } \\
\text { [PVL+, Tst1-] ST80-IVc (33\%) } \\
\text { [PVL-, Tst1-] ST80-IVa (11\%) } \\
\text { ST22 (22\%) } \\
\text { [PVL-, Tst1+] ST22-IVa-t223 } \\
(22 \%)\end{array}$ & $\begin{array}{l}\text { ST5 (22\%) } \\
\text { [PVL-, Tst1-] ST5-IVc }(11 \%) \\
\text { [PVL-, Tst1-] ST5-VII }(11 \%) \\
\text { ST535 (11\%) } \\
\text { [PVL-, Tst1-] ST535-IVh }(11 \%)\end{array}$ & $\begin{array}{l}\text { Frantz-fanon nephrology } \\
\text { department and Amizour } \\
\text { Hospital in Bejaia (2010-12) }\end{array}$ \\
\hline
\end{tabular}

Supplementary table 3. Molecular Typing of Colonizing MRSA Strains in the MENA Region. Numerical values are rounded to the nearest whole digit.

NR: Not reported. ${ }^{\mathbf{A}}$ spa typing, and CC assortment was done for 15 randomly selected isolates out of 26 total MRSA isolates. ${ }^{\mathbf{B}}$ spa typing, and $\mathrm{CC}$ assortment was done for 15 randomly selected isolates out of 33 total MRSA isolates. 


\begin{tabular}{|c|c|c|c|c|}
\hline Ref & \# MRSA & \multicolumn{2}{|c|}{ Molecular typing (CC, Spa, MLST, PFGE) } & Study location (study date) \\
\hline \multicolumn{5}{|c|}{ KSA } \\
\hline [66] & $120^{\mathbf{A}}$ & $\begin{array}{l}\text { Spa-CC037 (55\%): } \\
\text { t037 (35\%) } \\
\text { t030, t363 (5\% each) } \\
\text { t631 (3\%) } \\
\text { t019, t388 (2\% each) } \\
\text { t138, t } 459, \text { t748, t932, t1070 (1\% each) } \\
\text { Spa-CC790 (8\%): } \\
\text { t032, t223 (2\% each) } \\
\text { t790, t4573, t7604, t8506, t8855 (1\% each) } \\
\text { Spa-CC376(21\%): } \\
\text { t044 (19\%) } \\
\text { t376, t8731 (1\% each) }\end{array}$ & $\begin{array}{l}\text { Spa-CC690 (4\%): } \\
\text { t690 }(2.5 \%) \\
\text { t729, t8507 (0.8\%) } \\
\text { Cluster 5 no founder (5\%): } \\
\text { t304 (4.2\%) } \\
\text { t701 }(0.8 \%) \\
\text { Singletons }(8 \%): \\
\text { t002 (4.2\%) } \\
\text { t3059 }(1.7 \%) \\
\text { t364, t2235 }(0.8 \%)\end{array}$ & $\begin{array}{l}\text { Armed Forces Hospital, } \\
\text { Riyadh (2010) }\end{array}$ \\
\hline [67] & 107 & 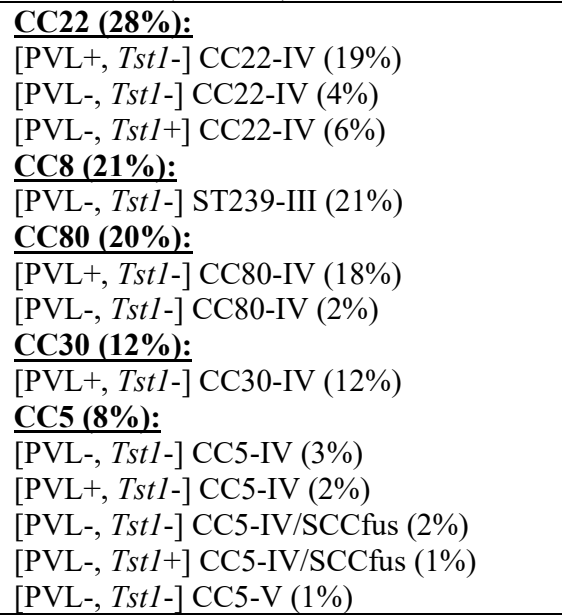 & 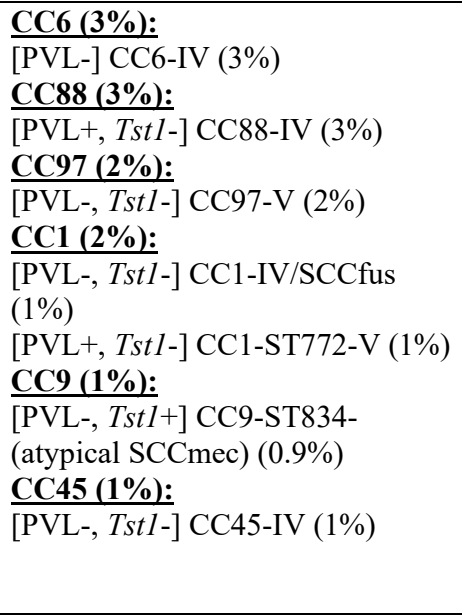 & $\begin{array}{l}\text { King Fahad Medical City, } \\
\text { Riyadh (NR) }\end{array}$ \\
\hline [72] & 101 & - & - & $\begin{array}{l}\text { Multiple hospitals ( } \mathrm{n}=\mathrm{NS}) \text {, } \\
\text { Jeddah (2009-2011) }\end{array}$ \\
\hline [38] & 100 & $\begin{array}{l}\text { PVL-] I }(3 \%) \\
\text { [PVL-] II }(9 \%) \\
\text { [PVL-] III }(39 \%) \\
\text { [PVL+] III }(8 \%)\end{array}$ & $\begin{array}{l}\text { PVL- }] \text { IV }(20 \%) \\
{[\text { PVL+] IV }(9 \%)} \\
\text { [PVL-] NT }(10 \%) \\
{[\text { PVL+] NT }(2 \%)}\end{array}$ & $\begin{array}{l}\text { Multiple hospitals }(\mathrm{n}=5), \\
\text { Makkah city }(2012)\end{array}$ \\
\hline [68] & 117 & 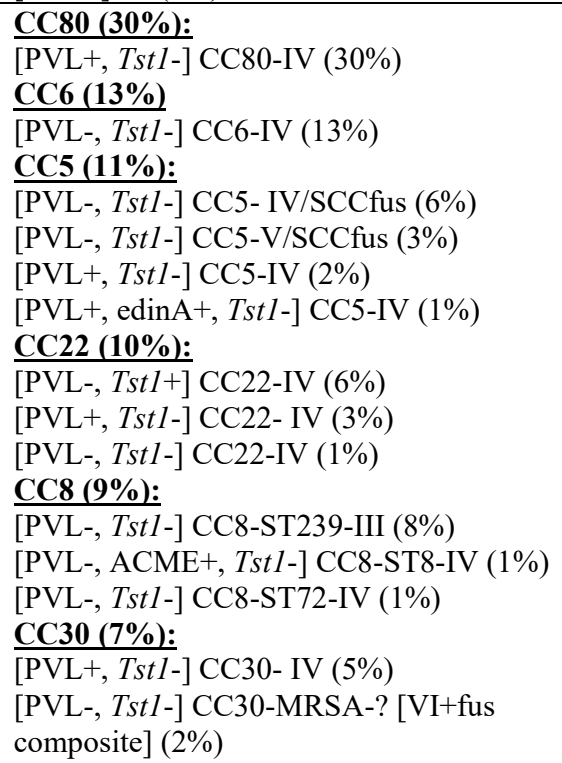 & 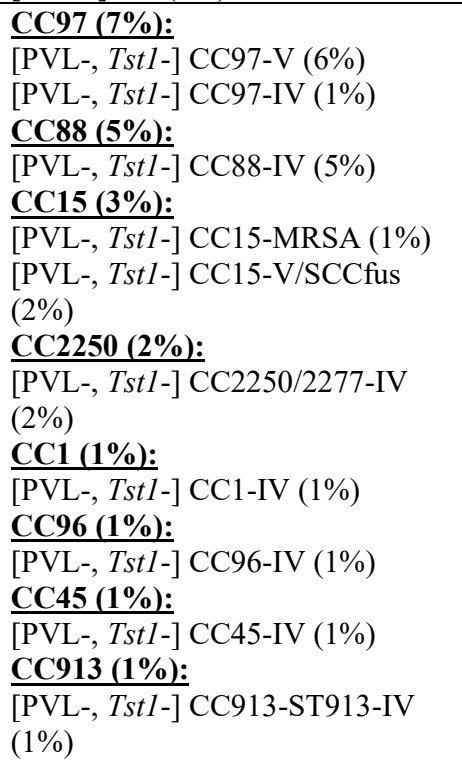 & $\begin{array}{l}\text { King Khalid University } \\
\text { Hospital, Riyadh (2009-15) }\end{array}$ \\
\hline [37] & 71 & $\begin{array}{l}\text { SCCmec I, II, and III (63\%): } \\
\text { [PVL-] I }(34 \%) \\
\text { [PVL-] III }(20 \%) \\
\text { [PVL-] II }(10 \%)\end{array}$ & $\begin{array}{l}\text { SCCmec IV, V, and NT (37\%): } \\
\text { [PVL+] IV }(17 \%) \\
\text { [PVL+] V }(10 \%) \\
\text { [PVL-] V }(6 \%) \\
\text { [PVL-] IV }(3 \%) \\
\text { [PVL+] NT }(1 \%)\end{array}$ & $\begin{array}{l}\text { Multiple hospitals, Taif city } \\
(2013-15)\end{array}$ \\
\hline & & & & \\
\hline
\end{tabular}




\begin{tabular}{|c|c|c|c|c|}
\hline [69] & $400^{\mathbf{B}}$ & $\begin{array}{l}\text { CC8 (58\%): } \\
\text { [PVL-] ST239-III-t030/t037/t421/t945/t860 } \\
\text { (46\%) } \\
\text { [PVL-] ST8-IV-t064 (1\%) } \\
\text { [PVL+] and [PVL-] ST8-IV-t008 (1\%) } \\
\text { [PVL-] ST8-V-t064 (1\%) } \\
\text { [PVL-] ST113-IV-t064 (1\%) } \\
\text { [PVL-] ST241-III-t1902/t037/t138 (9\%) } \\
\text { [PVL-] ST1465-III-t459 (0.3\%) } \\
\text { CC22 (9\%): } \\
\text { [PVL-, Tst1-] ST22-IV-t032/t790 } \\
\text { [PVL-, Tst1+] ST22-IV- } \\
\text { t223/t5708/t309/t3935/t2251/t852/t3107/t5983 } \\
\text { (9\%) } \\
\text { CC5 (9\%): } \\
\text { [PVL-, Tst1+] ST105-II-t002 (1\%) } \\
\text { [PVL-, Tst1-] ST5-II-t003/t242/t105 and } \\
\text { ST225-II-t045 (2\%) } \\
\text { [PVL+, Tst1-] ST5-IV-t306/t2164/t002/t688 } \\
\text { (3\%) } \\
\text { [PVL+, Tst1-] ST5-V-t688/t2164 (2\%) } \\
\text { [PVL+, Tst1-] ST5-VI-t688 (0.3\%) } \\
\text { [PVL+, Tst } 1-] \text { ST1637-V-t5258 (0.3\%) } \\
\text { [PVL-, Tst1-] ST149-IV-t1154/t2164 (1\%) } \\
\text { CC80 (7\%): } \\
\text { [PVL-, Tst1-] ST80-IV-t376/t8154 and } \\
\text { [PVL+, Tst1-] ST80-IV-t1154/t018/t042/t044 } \\
\text { (7\%) } \\
\text { CC30 (6\%): } \\
\text { [PVL+, Tst1-] ST30-IV-t019/t345/t318/t1130 } \\
\text { (4\%) } \\
\text { [PVL-, Tst1-] ST36-II-t018 and [PVL-, Tst1+] } \\
\text { ST36-II-t605 (2\%) }\end{array}$ & 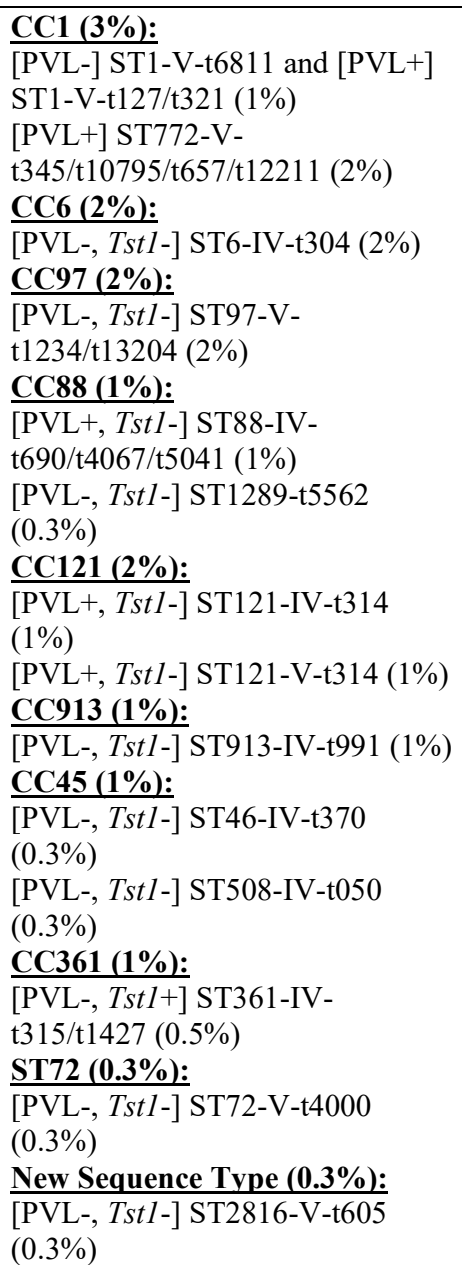 & $\begin{array}{l}\text { Multiple hospitals }(\mathrm{n}=13) \text {, } \\
\text { Kuwait. }(1992-2010)\end{array}$ \\
\hline [70] & $\begin{array}{l}26 \\
\text { SCCmec-IV }\end{array}$ & $\begin{array}{l}\text { CC80 (46\%): } \\
{[\text { PVL+] CC } 80-S T 80-I V(46 \%)} \\
\text { CC30 (31\%): } \\
{[\text { PVL+] CC30-ST30-IVc }(4 \%)} \\
{[\text { PVL+] CC30-ST30-IV }(27 \%)} \\
\text { CC5 (12\%): } \\
\text { [PVL-] CC5-ST5-IV (8\%) } \\
\text { [PVL-] CC5-ST6-IV (4\%) }\end{array}$ & $\begin{array}{l}\text { CC8 (8\%): } \\
\text { [PVL-]CC8-ST8-IVa (4\%) } \\
\text { [PVL-] CC8-ST8-IV (4\%) } \\
\text { CC undetermined (4\%): } \\
\text { [PVL-] ST361-IV (4\%) }\end{array}$ & $\begin{array}{l}\text { Multiple hospitals }(=7) \text {, } \\
\text { Kuwait. }(2001-3)\end{array}$ \\
\hline [82] & $\begin{array}{l}\text { 69 ST80- } \\
\text { SCCmec-IV }\end{array}$ & $\begin{array}{l}\text { ST80 (51\%): } \\
{[\text { PVL+, tst-]-ST80-IV }(35 \%)} \\
{[\text { PVL+, tst-] ST80-IVa }(1 \%)} \\
{[\text { PVL+, tst-] ST80-IVc }(1 \%)} \\
{[\text { PVL+, Tst } 1+]-\text { ST } 80-I V(2 \%)} \\
{[\text { PVL-, Tst } 1-]-\text { ST80-IV }(13 \%)}\end{array}$ & & $\begin{array}{l}\text { Multiple hospitals }(\mathrm{n}=8) \text {, } \\
\text { Kuwait. }(2005-6)\end{array}$ \\
\hline [84] & 6,922 & $\begin{array}{l}\text { SCCmec IV, V, NT (70\%): } \\
\text { IV (positive for urease production) }(46 \%) \\
\text { IV (negative for urease production) }(8 \%) \\
\text { V }(15 \%) \\
\text { NT }(1 \%)\end{array}$ & $\begin{array}{l}\text { SCCmec I, II, III (30\%): } \\
\text { I }(0.3 \%) \\
\text { II }(1 \%) \\
\text { III }(29 \%)\end{array}$ & $\begin{array}{l}\text { Multiple hospitals }(\mathrm{n}=14) \text {, } \\
\text { Kuwait (2011-15) }\end{array}$ \\
\hline [78] & $\begin{array}{l}37 \\
\text { EMRSA-15 } \\
\text { ST22 } \\
\text { Urease (-) }\end{array}$ & $\begin{array}{l}\text { \{UK-EMRSA-15/Barnim EMRSA } \mathbf{( 1 6 \% ) :} \\
\text { [PVL-, Tst } 1 \text {-] ST22-IVh-t032 (8\%) } \\
\text { [PVL-, Tst } 1 \text {-] ST22-IVa-t790 (8\%) } \\
\text { \{UK EMRSA-15/ [Tst1+] Middle-Eastern } \\
\text { variant\} (62\%): } \\
\text { [PVL-, Tst } 1+] \text { ST22-IVa-t223 }(51 \%) \\
\text { [PVL-, Tst } 1+] \text { ST22-IVa-t309 (3\%) } \\
\text { [PVL-, Tst } 1+] \text { ST22-IVa-t2251 (3\%) } \\
\text { [PVL-, Tst } 1+] \text { ST22-IVa-t3935 (3\%) }\end{array}$ & $\begin{array}{l}{[\text { PVL-, Tst1+] ST22-IVa-t5708 }} \\
(3 \%) \\
\{[\text { PVL+] CC22-MRSA-IV } \\
\text { variant\} (22\%): } \\
\text { PVL+, Tst1-] ST22-IV-t852 } \\
(14 \%) \\
{[\text { PVL+, Tst1-] ST22-IV-t3107 }} \\
(5 \%) \\
{[\text { PVL+, Tst } 1-] \text { ST22-IV-t5983 }} \\
(3 \%)\end{array}$ & $\begin{array}{l}\text { Multiple hospitals }(\mathrm{n}=10) \text {, } \\
\text { Kuwait }(2005-10)\end{array}$ \\
\hline
\end{tabular}




\begin{tabular}{|c|c|c|c|c|}
\hline [73] & $\begin{array}{l}102 \text { novel } \\
\text { MRSA } \\
\text { variants } \\
\text { from 1,327 } \\
\text { MRSA }\end{array}$ & 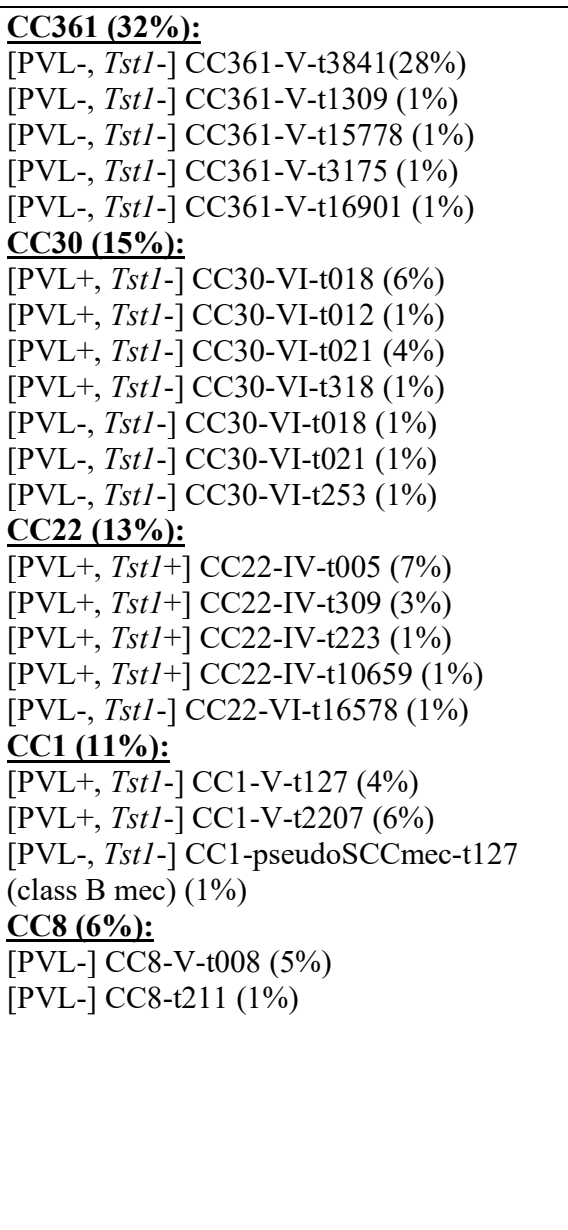 & 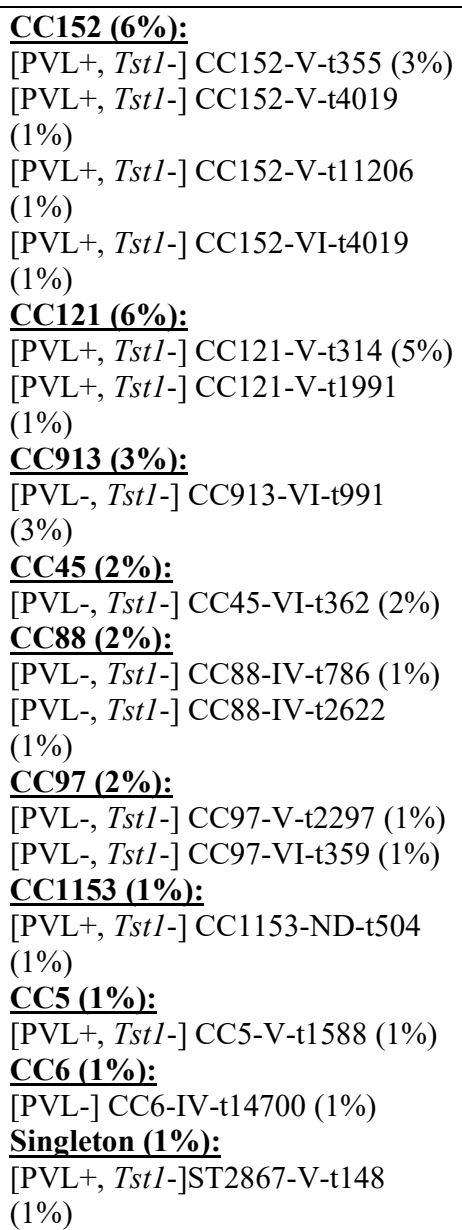 & $\begin{array}{l}\text { Multiple hospitals }(\mathrm{n}=13) \text {, } \\
\text { Kuwait (2016) }\end{array}$ \\
\hline [7] & $209^{\mathrm{C}}$ & $\begin{array}{l}\text { CC8 (27\%): } \\
\text { [PVL-] ST239-III-t860 (13\%) } \\
\text { [PVL-] ST239-III-t945 (8\%) } \\
\text { [PVL-[ ST239-III-t037 (1\%) } \\
\text { [PVL-] ST239-III-t713 (1\%) } \\
\text { [PVL-] ST239-III-t425 (1\%) } \\
\text { [PVL-] ST239-III-t1247 (1\%) } \\
\text { CC5 (18\%): } \\
\text { CC5-VI+SCCfus (5\%) } \\
\text { CC5-V+SCCfus (WA-MRSA-14/109) (4\%) } \\
\text { CC1 (9\%): } \\
\text { [PVL+] CC1-V+SCCfus (4\%) } \\
\text { CC6 (9\%): } \\
\text { CC6-IV (WA-MRSA-51) (8\%) } \\
\text { CC22 (9\%): } \\
\text { [PVL+, tst+] CC22-IV (3\%) } \\
\text { [PVL+, tst-] CC22-IV (2\%) }\end{array}$ & 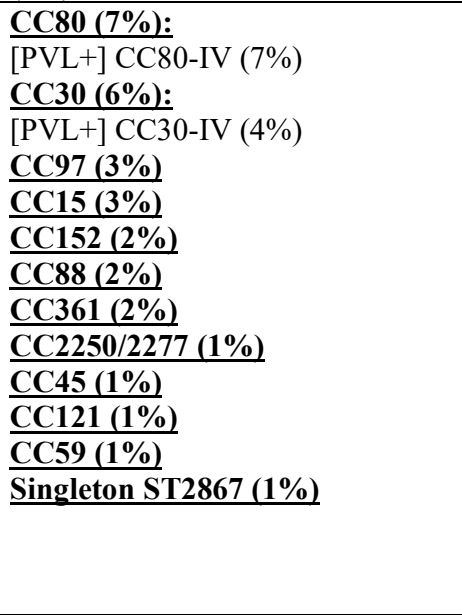 & $\begin{array}{l}\text { Farwaniya Hospital, } \\
\text { Farwaniyah (2016) }\end{array}$ \\
\hline \multicolumn{5}{|c|}{ UAE } \\
\hline [71] & $\begin{array}{l}26 \text { (year } \\
2003)\end{array}$ & 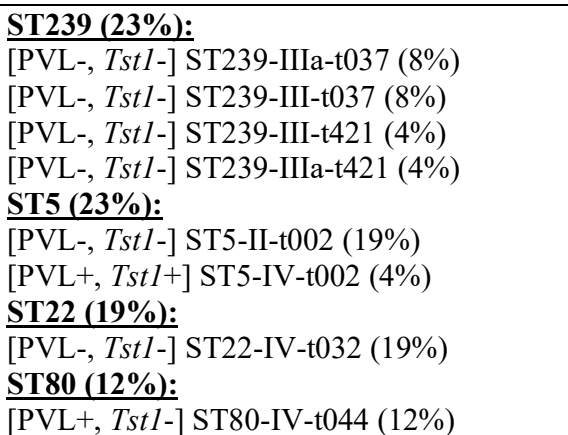 & $\begin{array}{l}\text { ST88 (8\%): } \\
{[\text { PVL+, Tst } 1-] \text { ST88-IV-t690 (8\%) }} \\
\text { ST30 (4\%): } \\
{[\text { PVL+, Tst1-] ST30-IV-t019 (4\%) }} \\
\text { ST97 (4\%): } \\
{[\text { PVL-, Tst1-] ST97-IV-t359 (4\%) }} \\
\text { ST113 (4\%): } \\
\text { [PVL-, Tst1-] ST113-IVa-t064 } \\
(4 \%) \\
\text { ST779 (4\%): } \\
\text { PVL-, Tst1-] ST779-IV-t878 (4\%) }\end{array}$ & $\begin{array}{l}\text { Tawam Hospital, Al Ain } \\
(2003-8)\end{array}$ \\
\hline
\end{tabular}




\begin{tabular}{|c|c|c|c|c|}
\hline & $\begin{array}{l}26 \text { (year } \\
2008)\end{array}$ & 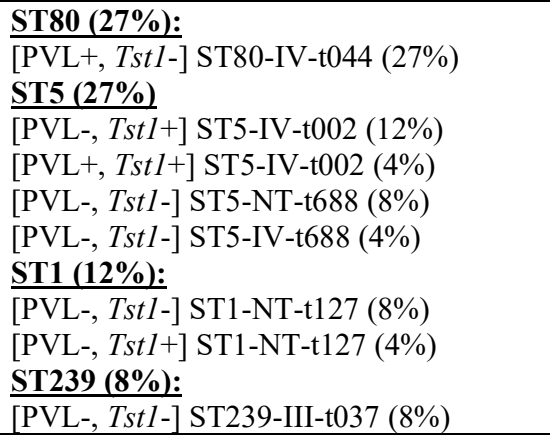 & $\begin{array}{l}\text { ST30 (8\%): } \\
{[\text { PVL+, Tst1-] ST30-IV-t018 (4\%) }} \\
\text { [PVL+, Tst1-] ST30-IV-t019 (4\%) } \\
\text { ST6 (8\%): } \\
\text { [PVL-, Tst1-] ST6-IV-t304 (8\%) } \\
\text { ST22 (4\%): } \\
\text { PVL-, Tst1-] ST22-NT-t005 (4\%) } \\
\text { ST45 (4\%): } \\
{[\text { PVL-, Tst1-] ST45-IV-t1081 (4\%) }} \\
\frac{\text { ST361 (4\%): }}{[\text { PVL-, Tst1-] ST361-IV-t315 (4\%) }}\end{array}$ & \\
\hline [87] & $57 \mathrm{CO}$ & $\frac{\text { SCCmec IV (100\%): }}{[\mathrm{PVL}+] \mathrm{IV}(100 \%)}$ & & $\begin{array}{l}\text { Al Qassimi hospital, Sharjah } \\
(2011-12)\end{array}$ \\
\hline \multicolumn{5}{|c|}{ Qatar } \\
\hline [11] & 61 & $\begin{array}{l}\text { ST30 (28\%): } \\
{[\text { PVL+] ST30-IV-t019 (28\%) }} \\
\text { ST5 (21\%): } \\
{[\text { PVL-] ST5-IV-t002 (21\%) }} \\
\text { ST80 (13\%): } \\
\text { PVL+] ST80-IV-t044 (13\%) } \\
\text { ST22 (13\%): } \\
\text { PVL+] ST22-IV-t852 (8\%) } \\
{[\text { PVL-] ST22-IV-t852 (5\%) }} \\
\text { ST8 (12\%): } \\
{[\text { PVL+, ACME+] ST8-IV-t008/t121 (7\%) }} \\
{[\text { PVL-] ST8-IV-spa-NT/t267 (5\%) }} \\
\text { ST15 (5\%): } \\
{[\text { PVL+] ST15-V-spa-NT/t657/7358 (5\%) }}\end{array}$ & $\begin{array}{l}\text { ST88 (3\%): } \\
\text { PVL+] ST88-IV-t690 (3\%) } \\
\text { ST-NT (3\%): } \\
\text { PVL-, ACME+] ST-NT-V-spa- } \\
\text { NT (2\%) } \\
\text { [PVL-] ST-NT-V-spa-NT (2\%) } \\
\text { ST121 (2\%): } \\
\text { PVL+] ST121-V-t314 (2\%) }\end{array}$ & $\begin{array}{l}\text { Al-Ahli Hospital, Doha (2009- } \\
\text { 10) }\end{array}$ \\
\hline \multicolumn{5}{|c|}{ 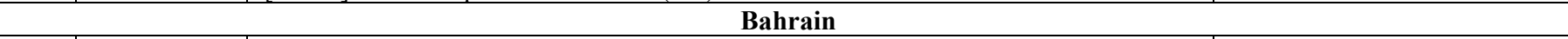 } \\
\hline [6] & 53 & $\begin{array}{l}\text { PFGE A }(\mathbf{8 3 \%} \%: \\
\text { SCCmec-III }(83 \%) \\
\text { PFGE C (6\%): } \\
\text { SCCmec-IV }(6 \%) \\
\text { PFGE D }(6 \%): \\
\text { SCCmec-IV }(6 \%)\end{array}$ & $\begin{array}{l}\text { PFGE B (2\%): } \\
\text { SCCmec-IV (2\%) } \\
\text { PFGE E (2\%): } \\
\text { SCCmec-IV }(2 \%)\end{array}$ & $\begin{array}{l}\text { Salmaniya Medical Complex, } \\
\text { Manama (2005) }\end{array}$ \\
\hline \multicolumn{5}{|c|}{ Gaza } \\
\hline [45] & 121 & 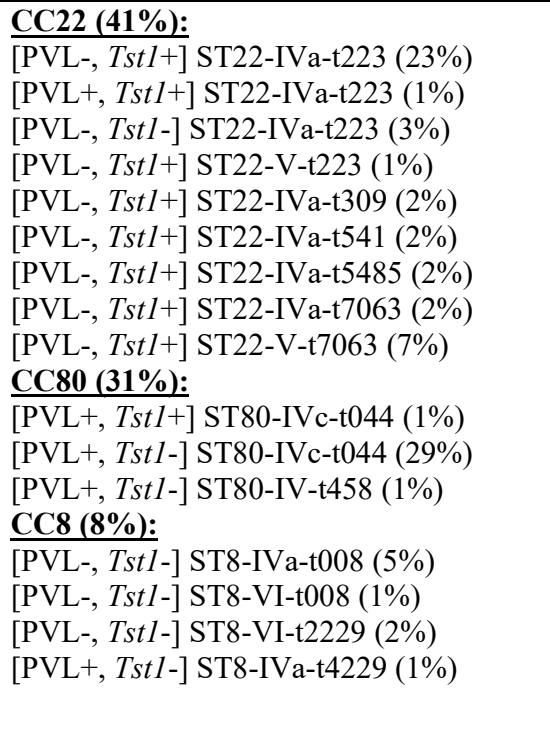 & 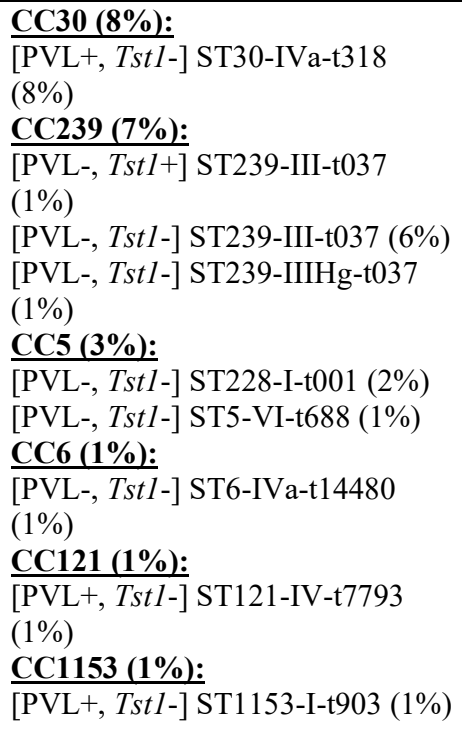 & $\begin{array}{l}\text { Al-Shifa Hospital, Gaza } \\
(2008-12)\end{array}$ \\
\hline
\end{tabular}




\begin{tabular}{|c|c|c|c|c|}
\hline [47] & 41 & 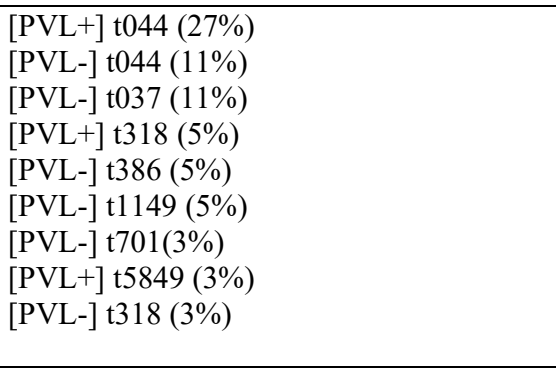 & $\begin{array}{l}\text { [PVL-] t6438 (3\%) } \\
\text { [PVL-] t091 (3\%) } \\
\text { [PVL-] t6432 (3\%) } \\
\text { [PVL-] t012 (3\%) } \\
\text { [PVL-] t743 (3\%) } \\
\text { [PVL+] t6439 (3\%) } \\
\text { [PVL-] t6303 (3\%) } \\
\text { [PVL-] t363 (3\%) } \\
{[\text { PVL-] t159 (3\%) }} \\
{[\text { PVL+] t5802 }(3 \%)}\end{array}$ & $\begin{array}{l}\text { Jordan University Hospital, } \\
\text { Amman }(2009-10)\end{array}$ \\
\hline [81] & $\begin{array}{l}31 \text { Spa- } \\
\text { CC044 } \\
\text { MRSA }\end{array}$ & $\begin{array}{l}\text { CC80 (100\%): } \\
\text { [PVL+, Tst } 1 \text {-] CC80-ST80-IV-t044 (19\%) } \\
\text { [PVL-, Tst } 1+] \text { CC80-ST80-IV-t044 (29\%) } \\
\text { [PVL+, Tst } 1+] \text { CC80-ST80-IV-t044 (19\%) } \\
\text { [PVL-, Tst } 1 \text { - ] CC80-ST80-IV-t044 (13\%) }\end{array}$ & $\begin{array}{l}\text { [PVL+, Tst1+] CC80-ST997-IV- } \\
\text { t044 (3\%) } \\
\text { [PVL-, Tst1+] CC80-ST80-IV- } \\
\text { t131 (3\%) } \\
\text { [PVL-, Tst1+] CC80-ST80-IV- } \\
\text { t5849 (3\%) } \\
\text { [PVL+, Tst1-] CC80-ST80-IV- } \\
\text { t5849 (3\%) } \\
\text { [PVL+, Tst1-] CC80-ST80-IV- } \\
\text { t5802 (3\%) } \\
\text { [PVL-, Tst1-] CC80-ST80-IV- } \\
\text { t6438 (3\%) }\end{array}$ & $\begin{array}{l}\text { Jordan University Hospital, } \\
\text { Amman (2000-11) }\end{array}$ \\
\hline \multicolumn{5}{|c|}{ Lebanon } \\
\hline [48] & 93 & $\begin{array}{l}\text { CC80 (55\%): } \\
\text { [PVL+] ST80-IVc-t044 (53\%) } \\
\text { [PVL+] ST80-IVc-t131 (2\%) } \\
\text { CC8 (15\%): } \\
\text { [PVL-] ST239-III-t037/t030 (11\%) } \\
\text { [PVL-] ST8-IVc-t008 (4\%) } \\
\text { CC97 (4\%): } \\
\text { [PVL-] ST97-V-t267 (4\%) }\end{array}$ & $\begin{array}{l}\text { CC6 (3\%): } \\
\text { [PVL-] ST6-IVc-t304 (3\%) } \\
\text { CC22 (3\%): } \\
\text { PVL-] ST22-IVc-t032 (3\%) } \\
\text { CC5 (1\%): } \\
\text { PVL-] ST5-IVc-t311 (1\%) } \\
\text { CC30 (1\%): } \\
{[\text { PVL+] ST30-IVc-t318 (1\%) }}\end{array}$ & $\begin{array}{l}\text { American University of Beirut } \\
\text { Medical Center, Beirut, } \\
\text { Lebanon. (2006-7) }\end{array}$ \\
\hline [81] & $\begin{array}{l}63 \text { spa- } \\
\text { CC044 }\end{array}$ & $\begin{array}{l}\text { CC80 (100\%): } \\
\text { [PVL+, Tst } 1 \text {-] ST80-IV-t044 (79\%) } \\
\text { [PVL-, Tst1-] ST80-IV-t044 (6\%) } \\
\text { [PVL+, Tst } 1 \text {-] ST80-IV-t131 (8\%) }\end{array}$ & $\begin{array}{l}\text { [PVL+, Tst1-] ST80-IV-t4222 } \\
(2 \%) \\
\text { [PVL+, Tst1-] ST80-IV-t6438 } \\
(2 \%) \\
\text { [PVL-, Tst1-] ST80-IV-t021 (2\%) } \\
\text { [PVL+, Tst1-] ST80-IV-t9135 } \\
(2 \%)\end{array}$ & $\begin{array}{l}\text { American University of Beirut } \\
\text { Medical Center, Beirut, } \\
\text { Lebanon. (2000-11) }\end{array}$ \\
\hline \multicolumn{5}{|c|}{ Iraq } \\
\hline [74] & $182^{\mathbf{D}}$ & $\begin{array}{l}\text { USA300 }(80 \%): \\
{[\mathrm{PVL}+] \text { IV }(78 \%)} \\
{[\mathrm{PVL}+] \text { MB }(2 \%)} \\
{[\mathrm{PVL}-] \text { IV }(1 \%)} \\
\text { USA100 }(4 \%): \\
{[\mathrm{PVL}+] \mathrm{NT}(3 \%)} \\
{[\mathrm{PVL}+] \mathrm{IV}(1 \%)} \\
\text { USA1100 }(3 \%): \\
{[\mathrm{PVL}+] \text { IV }(3 \%)} \\
\text { USA400 }(2 \%): \\
{[\mathrm{PVL}+] \text { IV }(2 \%)} \\
\text { USA800 }(1.6 \%): \\
{[\mathrm{PVL}+] \text { IV }(2 \%)}\end{array}$ & $\begin{array}{l}\text { USA1000 }(1 \%): \\
{[\text { PVL+] IV }(1 \%)} \\
\text { CSH1, CSH2, CSH3, CSH6, } \\
\text { CSH9, CSH10, and CSH11 }(1 \% \\
\text { each): } \\
{[\text { PVL+ }] \text { IV }(4 \%)} \\
\text { CSH4 }(0.5 \%), \text { CSH5 }(2 \%), \\
\text { CSH7(1\%): } \\
\text { PVL+] III (3\%) } \\
{[\text { PVL-] III (1\%) }} \\
\text { CSH8 (1\%): } \\
{[\text { PVL+] I (1\%) }} \\
\text { CSH5 (1\%): } \\
{[\text { PVL+] NT }(1 \%)}\end{array}$ & $\begin{array}{l}\text { Multiple combat support } \\
\text { hospitals }(n=3) \text {, Baghdad and } \\
\text { Al Anbar province (2007-9) }\end{array}$ \\
\hline \multicolumn{5}{|c|}{ Egypt } \\
\hline [55] & 12 & - & - & $\begin{array}{l}\text { Suez Canal University, } \\
\text { Ismailia (2013) }\end{array}$ \\
\hline$[56]$ & $18 \mathbf{C O}$ & - & - & $\begin{array}{l}\text { Alexandria University, } \\
\text { Alexandria (NR) }\end{array}$ \\
\hline [54] & 18 & $\begin{array}{l}{[\mathrm{PVL}+] \text { II }(33 \%)} \\
{[\mathrm{PVL}+] \text { IVd }(11 \%)} \\
{[\mathrm{PVL}+] \text { III }(11 \%)} \\
{[\mathrm{PVL}-] \text { V }(11 \%)} \\
{[\mathrm{PVL}+] \text { NT }(11 \%)} \\
\end{array}$ & $\begin{array}{l}{[\mathrm{PVL}+] \text { IVa }(6 \%)} \\
{[\mathrm{PVL}-] \mathrm{IVd}(6 \%)} \\
{[\mathrm{PVL}+] \mathrm{V}(6 \%)} \\
{[\mathrm{PVL}+] \mathrm{IVc}(6 \%)}\end{array}$ & $\begin{array}{l}\text { University Hospital and } \\
\text { Diagnostic Laboratories, } \\
\text { Zagazig and Assuit (2010-12) }\end{array}$ \\
\hline
\end{tabular}




\begin{tabular}{|c|c|c|c|c|}
\hline \multicolumn{5}{|c|}{ Algeria } \\
\hline [58] & 92 & $\begin{array}{l}\text { CC8 (78\%): } \\
\text { [PVL-] ST239-III-t037 (43\%) } \\
\text { [PVL-] ST239-III-t932 (35\%) }\end{array}$ & $\begin{array}{l}\text { CC80 (21\%): } \\
\text { PVL+] ST80-IVc-t044 (21\%) } \\
\text { CC22 (1\%): } \\
\text { [PVL-] ST22-IVc-t005 (1\%) }\end{array}$ & $\begin{array}{l}\text { Annaba Hospitals, Annaba } \\
\text { (2010) }\end{array}$ \\
\hline \multirow[t]{2}{*}{ [59] } & $15 \mathbf{H O}^{\mathbf{E}}$ & 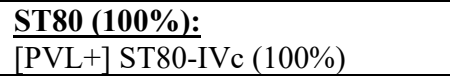 & & \multirow[t]{2}{*}{$\begin{array}{l}\text { Beni Messous University } \\
\text { Hospital, Algiers (2010-11) }\end{array}$} \\
\hline & $10 \mathbf{C O}^{\mathbf{E}}$ & $\begin{array}{l}\text { ST80 (90\%): } \\
\text { [PVL+] ST80-IVc (70\%) } \\
\text { [PVL-] ST80-IVc }(20 \%)\end{array}$ & $\frac{\text { ST39 (10\%): }}{\text { [PVL-] ST39-II (10\%) }}$ & \\
\hline \multirow[t]{2}{*}{ [61] } & $84 \mathbf{C O}^{\mathrm{F}, \mathrm{G}}$ & $\begin{array}{l}\text { ST80 (97\%): } \\
\text { [PVL+] ST80-IV-t044 (85\%) } \\
\text { [PVL+] ST80-IV-t4143 (3\%) } \\
\text { [PVL-] ST80-IV (3\%) } \\
\text { [PVL-] ST80-V }(6 \%)\end{array}$ & $\frac{\text { ST241 (3\%): }}{\text { [PVL-] ST241-III (3\%) }}$ & \multirow[t]{2}{*}{$\begin{array}{l}\text { Mustapha Bacha University } \\
\text { Hospital, Algiers (2006-7) }\end{array}$} \\
\hline & $137 \mathbf{H O}^{\mathbf{F}, \mathbf{G}}$ & $\begin{array}{l}\text { ST80 (77\%): } \\
\text { [PVL+] ST80-IV-t044 (75\%) } \\
\text { [PVL-] ST80-IV (2\%) }\end{array}$ & $\begin{array}{l}\text { CC80 (21\%): } \\
\text { [PVL+] ST80-IVc-t044 (21\%) } \\
\text { ST5 (11\%): } \\
\text { [PVL-] ST5-IV (11\%) }\end{array}$ & \\
\hline [76] & 64 & - & - & $\begin{array}{l}\text { Didouche Mourad Hospital, } \\
\text { Constantine Province (2005-7) }\end{array}$ \\
\hline [75] & 84 & $\begin{array}{l}\text { ST80 (86\%): } \\
\text { PVL+] ST80-ND }(2 \%) \\
\text { [PVL+] ST80-IV (83\%) } \\
\text { ST239 (8\%): } \\
\text { [PVL-] ST239-III }(8 \%)\end{array}$ & $\begin{array}{l}\text { Other (6\%): } \\
\text { [PVL-] IV (5\%) } \\
\text { [PVL-] II }(1 \%)\end{array}$ & $\begin{array}{l}\text { Bolghine Ibn Ziri University } \\
\text { hospital, Algiers (2006-11) }\end{array}$ \\
\hline [60] & $73^{\mathrm{H}}$ & $\frac{\text { ST239 (82\%): }}{\text { [PVL-, Tst1-] ST239 (82\%) }}$ & $\begin{array}{l}\text { ST80 (14\%): } \\
{[\text { PVL+, Tst } 1-] \text { ST80 (14\%) }} \\
\text { Unknown (4\%) } \\
{[\text { PVL-, Tst } 1-](4 \%)}\end{array}$ & $\begin{array}{l}\text { University Hospital Ibn } \\
\text { Rochd, Annaba (2011-12) }\end{array}$ \\
\hline \multicolumn{5}{|c|}{ Tunisia } \\
\hline [64] & 58 & 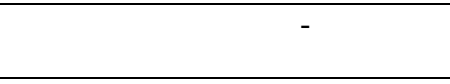 & 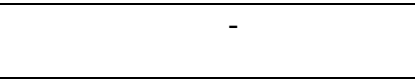 & $\begin{array}{l}\text { Charles Nicolle Hospital, } \\
\text { Tunis (2005-7) }\end{array}$ \\
\hline [65] & $\begin{array}{l}24 \\
\text { tetracycline- } \\
\text { resistant }\end{array}$ & $\begin{array}{l}\text { CC8 (79\%): } \\
\text { [PVL-, Tst } 1 \text {-] ST247-t052 (50\%) } \\
\text { [PVL-, Tst } 1 \text { - }] \text { ST239-t037 (21\%) } \\
\text { [PVL-, Tst } 1 \text { - }] \text { ST239-t14712 (4\%) } \\
\text { [PVL-, Tst } 1 \text {-] ST241-t129 (4\%) } \\
\text { CC80 (8\%): } \\
\text { [PVL-, Tst } 1 \text {-] ST728-t044 (4\%) } \\
\text { [PVL+, Tst } 1 \text { - ] ST728-t044 (4\%) }\end{array}$ & $\begin{array}{l}\text { CC5 (8\%): } \\
\text { PVL-, Tst1-] ST641-t311 (4\%) } \\
\text { [PVL-, Tst1-] ST5-t7144 (4\%) } \\
\text { CC398 (4\%): } \\
{[\text { PVL-, Tst1-] ST398-t899 (4\%) }}\end{array}$ & $\begin{array}{l}\text { Military Hospital of Tunis, } \\
\text { Tunis }(2011-12)\end{array}$ \\
\hline \multirow[t]{2}{*}{ [77] } & $28 \mathbf{C O}^{\mathbf{I}}$ & $\begin{array}{l}\text { CC80 (86\%): } \\
{[\mathrm{PVL}+] \text { ST } 80-\mathrm{IVc}(60 \%)} \\
{[\mathrm{PVL}+] \text { ST } 80-N T(7 \%)} \\
{[\mathrm{PVL}+] \text { ST153-IVc }(4 \%)} \\
{[\mathrm{PVL}+] \text { ST } 153-\mathrm{NT}(4 \%)} \\
{[\mathrm{PVL}+] \text { ST2563-IVc }(4 \%)} \\
{[\mathrm{PVL}-] \text { ST } 80-\mathrm{IVc}(7 \%)}\end{array}$ & $\begin{array}{l}\text { CC5 (7\%): } \\
\text { [PVL-] ST5-I (4\%) } \\
\text { [PVL-] ST5-IVc (4\%) } \\
\text { CC1 (4\%): } \\
\text { [PVL-] ST1-NT (4\%) } \\
\text { CC45 (4\%): } \\
\text { [PVL-] ST45-NT (4\%) }\end{array}$ & \multirow[t]{2}{*}{$\begin{array}{l}\text { Habib Bourguiba Hospital and } \\
\text { Charles Nicolle Hospital, } \\
\text { Tunis (2004-8) }\end{array}$} \\
\hline & $41 \mathbf{H O}^{\mathbf{I}}$ & $\begin{array}{l}\text { CC80 (51\%): } \\
\text { PVL+] ST80-IVc }(49 \%) \\
{[\text { PVL+] ST1440-IVc }(2 \%)} \\
\text { CC8 (32\%): } \\
{[\text { PVL-] ST239-III }(10 \%)} \\
{[\text { PVL-] ST241-III (7\%) }} \\
{[\text { PVL-] ST247-I (7\%) }} \\
{[\text { PVL-] ST1819-I (7\%) }}\end{array}$ & $\begin{array}{l}\text { CC5 (7\%): } \\
\text { [PVL-] ST5-IVc (5\%) } \\
\text { [PVL-] ST5-NT (2\%) } \\
\text { CC15 (4.9\%): } \\
\text { [PVL-] ST97-NT }(4.9 \%) \\
\text { CC1 (2\%): } \\
\text { PVL-] ST1-NT }(2 \%) \\
\text { CC22 (2\%): } \\
\text { [PVL-] ST22-NT (2\%) }\end{array}$ & \\
\hline
\end{tabular}

Supplementary Table 4. Molecular Typing of Infective MRSA Isolates in the MENA Region.

CO: CO-MRSA; HO: HO-MRSA; NR: not reported; NT: not typeable; S-: singleton; Spa-CC: spa cluster. When a sample is not designated "CO" or "HO" it indicates that the study does not distinguish site of acquisition. Values were rounded to the nearest whole number.

A 60 cancer and 60 noncancer patients, ${ }^{\text {B }}$ Percentages for each spa type was not provided in the study, ${ }^{\text {C }}$ In this study only 120 from the 209 isolates underwent DNA microarray analysis; these isolates represented the 56 spa types found. The MLST types were only reported for CC8 and S-ST2867, ${ }^{\mathbf{D}}$ Isolates from USA service members deployed to Iraq. Sixteen strains displayed 11 unique pulsotypes not previously described and were labeled CSH1-CSH11, ${ }^{\mathbf{E}}$ subjects were neonates. In this study, outpatients and inpatients $<48$ hrs from admission were considered CO-MRSA while inpatients $>48$ hrs. post admission were HO-MRSA. Other epidemiological risk factors for HO-MRSA were not specified, 
F This study distinguished between CO-MRSA and HO-MRSA by the epidemiological criteria specified in text, ${ }^{\mathbf{G}}$ spa typing was done for 46 PVL [+] isolates only and revealed only 2 spa types: t044 and t4143, ${ }^{\mathbf{H}}$ Diabetic foot infections, ${ }^{\mathbf{I}}$ Criteria used to distinguish between HOMRSA and CO-MRSA is not specified. 


\begin{tabular}{|c|c|c|c|c|c|c|c|c|c|c|c|c|c|c|c|c|}
\hline Country & $\begin{array}{c}\text { Ref } \\
\text { (date) }\end{array}$ & $\begin{array}{c}\# \\
\text { MRSA } \\
\end{array}$ & Rif & FA & $\mathrm{Cl}$ & $\mathrm{TS}$ & Cip & Tet & Dox & EM & Vanc & Lin & Teic & Dap & Mup & Gen \\
\hline \multicolumn{17}{|c|}{ Colonizing MRSA strains } \\
\hline Oman $^{A}$ & $\begin{array}{c}{[21,} \\
22] \\
(\mathrm{NR})\end{array}$ & $34 \mathrm{CV}$ & 100 & - & 76 & - & - & - & 88 & 50 & 88 & 100 & 82 & - & - & - \\
\hline $\mathrm{Oman}^{\mathrm{A}}$ & $\begin{array}{c}{[21,} \\
22] \\
(\mathrm{NR})\end{array}$ & $\begin{array}{c}49 \\
\mathrm{HCW}\end{array}$ & 96 & - & 65 & - & - & - & 92 & 51 & 88 & 100 & 84 & - & - & - \\
\hline $\mathrm{KSA}^{\mathrm{A}}$ & $\begin{array}{c}{[12]} \\
(2011) \\
\end{array}$ & $\begin{array}{c}26 \text { OP } \\
\mathbf{C O}^{\mathbf{B}}\end{array}$ & 96 & - & 89 & 54 & 100 & - & - & 42 & 96 & - & - & - & - & 73 \\
\hline $\mathrm{KSA}^{\mathrm{A}}$ & $\begin{array}{c}14] \\
(2014- \\
15) \\
\end{array}$ & $48 \mathrm{IP}$ & 58 & - & 13 & 33 & 12 & 29 & - & 15 & $98^{\mathrm{C}}$ & 67 & 54 & - & - & 92 \\
\hline $\mathrm{KSA}^{\mathrm{A}}$ & $\begin{array}{c}{[19]} \\
(2012- \\
14) \\
\end{array}$ & $\begin{array}{c}36 \\
\text { HCWs }\end{array}$ & - & - & 3 & 83 & 83 & 69 & - & 3 & $100^{\mathrm{E}}$ & 100 & - & - & - & - \\
\hline Lebanon $^{\mathrm{A}}$ & $\begin{array}{c}{[29]} \\
(2006- \\
7) \\
\end{array}$ & $8 \mathrm{CV}$ & - & - & - & 100 & 100 & - & - & - & 100 & - & - & - & 100 & 100 \\
\hline $\mathrm{Gaza}^{\mathrm{D}}$ & $\begin{array}{c}{[23]} \\
(2009) \\
\end{array}$ & $94 \mathrm{CV}$ & - & 95 & 64 & 100 & 99 & - & - & 62 & 100 & - & - & - & - & 97 \\
\hline $\mathrm{Gaza}^{\mathbf{A}}$ & $\begin{array}{c}{[25]} \\
(2015)\end{array}$ & $\begin{array}{c}51 \\
\mathrm{HCW}\end{array}$ & $88 \%$ & - & $86 \%$ & - & $88 \%$ & $86.3 \%$ & & $65 \%$ & $84 \%$ & - & - & - & - & $92 \%$ \\
\hline Jordan $^{\mathrm{F}}$ & $\begin{array}{c}{[27]} \\
(2011- \\
12) \\
\end{array}$ & $26 \mathrm{CV}$ & 100 & 96 & 100 & - & 100 & - & - & 42 & 100 & 100 & 100 & 100 & 100 & 100 \\
\hline Jordan $^{\mathrm{F}}$ & $\begin{array}{c}27] \\
(2011- \\
12) \\
\end{array}$ & $\begin{array}{c}30 \\
\mathrm{HCW}\end{array}$ & 100 & 90 & 90 & - & 93 & - & - & 42 & 100 & 100 & 100 & 100 & 100 & 100 \\
\hline Egypt $^{\mathrm{A}}$ & $\begin{array}{c}{[12]} \\
(2011) \\
\end{array}$ & $\begin{array}{c}33 \mathrm{OP} \\
\mathbf{C O}^{\mathbf{B}}\end{array}$ & 91 & - & 67 & 52 & 100 & - & - & 45 & 91 & - & - & - & - & 67 \\
\hline Algeria $^{\mathrm{A}}$ & $\begin{array}{c}{[34]} \\
(2010- \\
12) \\
\end{array}$ & 9 IP & 100 & - & 100 & 78 & 100 & 67 & - & 89 & 100 & 100 & 100 & - & - & 67 \\
\hline Kuwait $^{\mathbf{A}}$ & $\begin{array}{c}20] \\
(2005- \\
7) \\
\end{array}$ & $18 \mathrm{IP}$ & $100 \%$ & $33 \%$ & $33 \%$ & $72 \%$ & $28 \%$ & $28 \%$ & & $33 \%$ & $100 \%$ & $100 \%$ & $100 \%$ & - & $95 \%$ & $28 \%$ \\
\hline \multicolumn{17}{|c|}{ Infective MRSA Strains } \\
\hline $\mathrm{KSA}^{\mathbf{A}}$ & $\begin{array}{c}{[85]} \\
(2004- \\
5) \\
\end{array}$ & $\begin{array}{l}397 \\
\text { NS }\end{array}$ & - & 4 & - & 21 & - & - & - & - & $100^{\mathbf{E}}$ & 97 & - & - & 74 & 30 \\
\hline $\mathrm{KSA}^{\mathbf{A}}$ & $\begin{array}{c}{[85]} \\
(2004- \\
5) \\
\end{array}$ & $\begin{array}{l}115 \\
\text { NS }\end{array}$ & - & 6 & - & 77 & - & - & - & - & $100^{\mathbf{E}}$ & 91 & - & - & 89 & 74 \\
\hline $\mathrm{KSA}^{\mathrm{NS}}$ & $\begin{array}{c}66] \\
(2010)\end{array}$ & $\begin{array}{l}120 \\
\text { NS } \\
\end{array}$ & 68 & 45 & 57 & 2 & - & - & - & 38 & 100 & - & - & - & 68 & - \\
\hline $\mathrm{KSA}^{\mathbf{A}}$ & $\begin{array}{c}72] \\
(2009- \\
11) \\
\end{array}$ & $\begin{array}{l}101 \\
\text { NS }\end{array}$ & 87 & 71 & 67 & - & 62 & 57 & - & 56 & 100 & 83 & 88 & - & 88 & 71 \\
\hline Kuwait $^{\mathbf{A}}$ & $\begin{array}{c}84] \\
(2011- \\
15) \\
\end{array}$ & $\begin{array}{c}6,922 \\
\mathrm{NS}\end{array}$ & 99 & 59 & 58 & 66 & 57 & 61 & - & 58 & $99^{\mathrm{E}}$ & 100 & 97 & - & 95 & 61 \\
\hline Kuwait $^{\mathbf{A}}$ & $\begin{array}{c}{[73]} \\
(2016) \\
\end{array}$ & $\begin{array}{c}1,327 \\
\mathrm{NS} \\
\end{array}$ & 100 & 53 & 60 & 60 & 61 & 68 & - & 60 & $100^{\mathrm{E}}$ & 100 & 100 & - & 96 & - \\
\hline Kuwait $^{\mathbf{A}}$ & $\begin{array}{c}{[7]} \\
(2016) \\
\end{array}$ & $\begin{array}{l}209 \\
\text { NS } \\
\end{array}$ & 100 & 36 & 48 & 55 & 50 & 45 & - & 48 & $100^{\mathbf{E}}$ & 100 & 100 & - & 98 & 45 \\
\hline Oman $^{\mathbf{A}}$ & $\begin{array}{c}43] \\
(2016- \\
17) \\
\end{array}$ & $\begin{array}{l}733 \\
\text { NS }\end{array}$ & - & - & 78 & 94 & 69 & - & - & 71 & 100 & 100 & - & - & - & 85 \\
\hline Bahrain $^{\mathbf{A}}$ & $\begin{array}{c}{[6]} \\
(2005)\end{array}$ & $53 \mathrm{NS}$ & 100 & 8 & 13 & 11 & 8 & 11 & - & 9 & $100^{\mathbf{E}}$ & 100 & 100 & - & 89 & 26 \\
\hline Lebanon $^{\mathbf{A}}$ & $\begin{array}{c}{[79]} \\
(2011)\end{array}$ & $39 \mathrm{NS}$ & 100 & - & 87 & 97 & 87 & 69 & - & 80 & 100 & - & 100 & - & - & - \\
\hline $\mathrm{Gaza}^{\mathbf{G}}$ & $\begin{array}{c}45] \\
(2008- \\
12) \\
\end{array}$ & $\begin{array}{l}121 \\
\text { NS }\end{array}$ & 92 & - & 77 & 80 & - & 68 & - & 71 & 100 & 100 & 100 & 100 & 100 & 80 \\
\hline
\end{tabular}




\begin{tabular}{|c|c|c|c|c|c|c|c|c|c|c|c|c|c|c|c|c|}
\hline Jordan $^{\mathbf{A}}$ & $\begin{array}{c}46] \\
(2008- \\
12) \\
\end{array}$ & $\begin{array}{l}113 \\
\text { NS }\end{array}$ & 86 & - & 55 & 58 & - & 58 & - & 23 & 100 & 97 & 97 & - & - & 47 \\
\hline $\operatorname{Iraq}^{\mathrm{NS}}$ & $\begin{array}{c}{[74]} \\
(2007- \\
9)\end{array}$ & $\begin{array}{c}182 \\
\mathrm{NS}^{\mathrm{H}}\end{array}$ & 95 & - & 77 & 91 & - & 91 & - & 10 & 100 & 96 & - & - & - & 87 \\
\hline $\operatorname{Iraq}^{\mathrm{NS}}$ & $\begin{array}{c}{[51]} \\
(2005- \\
9)\end{array}$ & $\begin{array}{c}303 \\
\mathrm{NS}^{\mathrm{H}}\end{array}$ & 91 & - & 75 & 91 & 49 & 80 & - & 15 & 100 & - & - & - & - & 89 \\
\hline $\operatorname{Iraq}^{\mathbf{A}}$ & $\begin{array}{c}52] \\
(2008)\end{array}$ & $\begin{array}{c}15 \\
\mathrm{NS}^{\mathrm{H}}\end{array}$ & 100 & - & 87 & 100 & 73 & - & - & 13 & & - & - & - & - & - \\
\hline Egypt $^{\mathbf{A}}$ & $\begin{array}{c}{[57]} \\
(2005- \\
13)\end{array}$ & $\begin{array}{c}21 \\
\mathbf{C O}^{\mathbf{I}}\end{array}$ & 86 & - & 100 & 81 & 91 & 14 & & 81 & 100 & - & - & - & - & 91 \\
\hline Egypt $^{\mathbf{A}}$ & $\begin{array}{c}{[57]} \\
(2005- \\
13) \\
\end{array}$ & $\begin{array}{c}343 \\
\text { HO }^{\mathbf{I}}\end{array}$ & 80 & - & 44 & 83 & 30 & 16 & & 36 & 100 & - & - & - & - & 19 \\
\hline Algeria $^{\mathbf{A}}$ & $\begin{array}{c}{[75]} \\
(2006- \\
11)\end{array}$ & $84 \mathrm{NS}$ & 100 & 46 & 91 & 76 & - & 85 & & 42 & - & 100 & - & 100 & - & - \\
\hline Algeria $^{\mathrm{A}}$ & $\begin{array}{c}{[59]} \\
(2010- \\
11)\end{array}$ & $\begin{array}{c}10 \\
\mathbf{C O}^{\mathbf{I}}\end{array}$ & 100 & - & 80 & 100 & 70 & 40 & - & 70 & 100 & 100 & 100 & - & - & 100 \\
\hline Algeria $^{\mathbf{A}}$ & $\begin{array}{c}59] \\
(2010- \\
11)\end{array}$ & $\begin{array}{c}15 \\
\mathrm{HO}^{\mathbf{I}}\end{array}$ & 100 & - & 93 & 100 & 87 & 13 & - & 73 & 100 & 100 & 100 & - & - & 100 \\
\hline Algeria $^{\mathbf{A}}$ & $\begin{array}{c}{[62]} \\
2007- \\
9\end{array}$ & $\begin{array}{c}165 \\
\mathbf{H O}^{\mathrm{B}}\end{array}$ & - & - & 88 & - & - & - & - & 44 & $98^{\mathrm{J}}$ & - & - & - & - & 70 \\
\hline Tunisia $^{\mathbf{A}}$ & $\begin{array}{c}{[77]} \\
(2004- \\
8)\end{array}$ & $69 \mathrm{NS}$ & 80 & - & 84 & 91 & 59 & 17 & - & 48 & 100 & - & 100 & - & - & 74 \\
\hline
\end{tabular}

Supplementary table 5. Antimicrobial Susceptibility Profile of MRSA Isolates in the Arab World. Presented values are percentages of tested isolates. Infective isolates are labeled as CO-MRSA or HO-MRSA when specified by the cited study, otherwise they are labeled as Not specified Cip: ciprofloxacin; Cl: clindamycin; CO: community-onset MRSA; CV: community volunteers; Dapt: daptomycin; EM: erythromycin; FA: fusidic acid; Gen: gentamicin; HCWs: health-care workers; HO: hospital-onset MRSA; IP: inpatient; Lin: linezolid; Mup: Mupirocin; NR: not reported; NS: not specified; OP: outpatients; Rif: Rifampicin; Tet: tetracycline; Teic: teicoplanin; TS: trimethoprim-sulfamethoxazole; Vanc: vancomycin.

${ }^{\text {A }}$ Antibiotic susceptibility testing (AST) by standard agar disk diffusion methodology according to clinical and laboratory standards institute guidelines, ${ }^{\mathrm{B}}$ CO-MRSA by epidemiological criteria described in text, ${ }^{\mathrm{C}}$ Non-susceptible strain identified as Vancomycin intermediate $S$. aureus (VISA), ${ }^{\mathrm{D}}$ AST determined using the VITEK-2 system, ${ }^{\mathrm{E}}$ AST to this antibiotic was tested via E-test method, ${ }^{\mathrm{F}}$ AST by standard agar disk diffusion and interpretation according to British society for antimicrobial chemotherapy method, ${ }^{\mathrm{G}}$ AST for 2003 done with VITEK2 system, AST for 2012 done with MicroScan WalkAway Plus System (Siemens Healthcare, Erlangen, Germany), ${ }^{\mathrm{H}}$ Combat support hospitals, ${ }^{\mathrm{I}}$ COMRSA was defined as infection diagnosed $<2-3$ days from admission, and HO-MRSA $>2-3$ days from admission, ${ }^{\mathrm{J}}$ Non-susceptible isolates identified as vancomycin resistant $S$. aureus (VRSA) as opposed to VISA 
Supplementary Figure 1. Illustrates how different typing techniques can be grouped together into a clonal complex.

Interconnection Between the Various Molecular Typing Techniques.

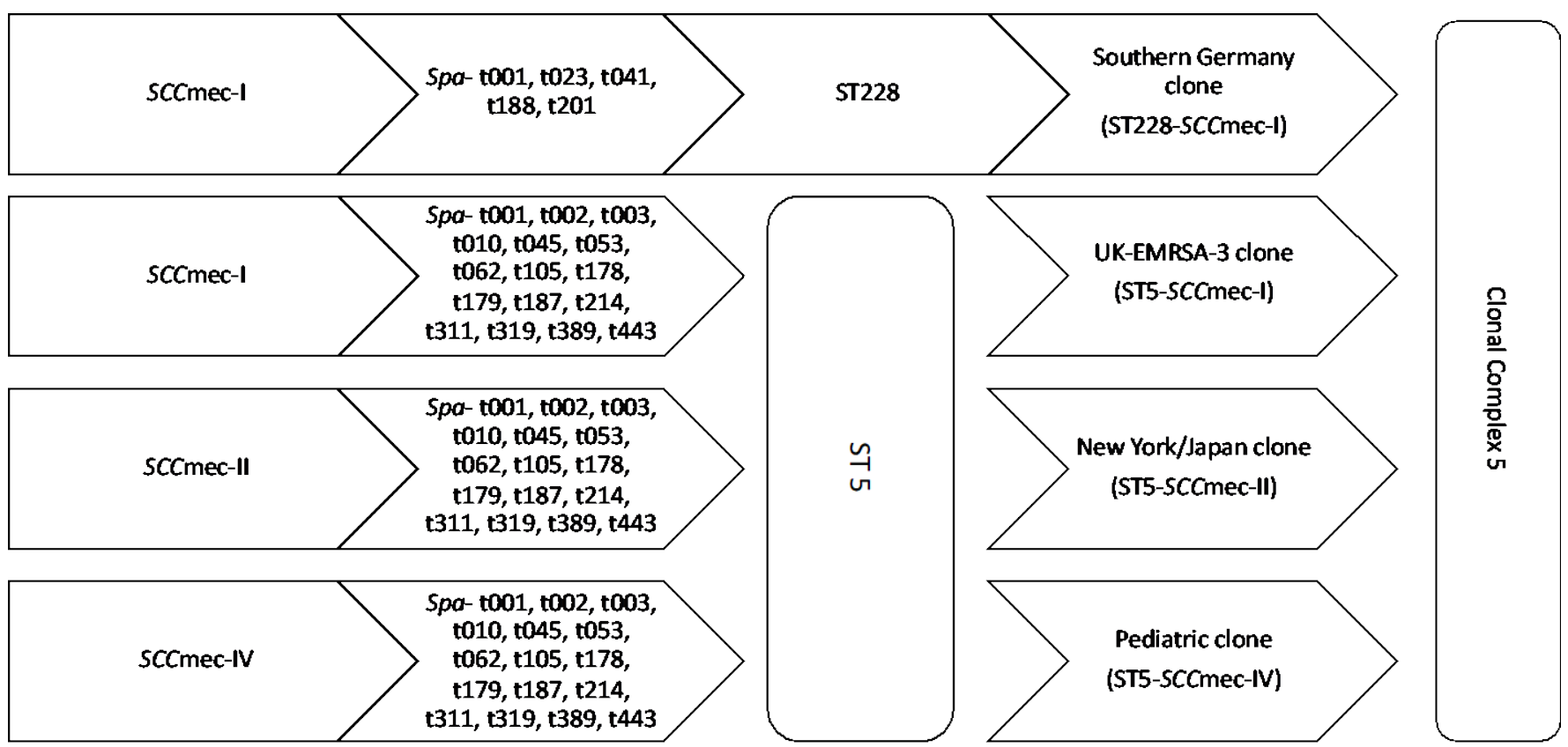

- One spa type can have multiple SCCmecs. One spa type can belong to multiple STs and multiple clones but only 1 clonal complex (CC).

- $\quad$ One ST can have multiple spa types and SCCmecs. One ST can belong to multiple clones but only $1 \mathrm{CC}$.

- Clone nomenclature depends on ST + SCCmec combination. Therefore, one clone can have only 1 ST and SCCmec combination. One clone can belong to only $1 \mathrm{CC}$.

- The highest level of classification is the CC. One CC can have multiple clones, STs, spa types and SCCmecs. 